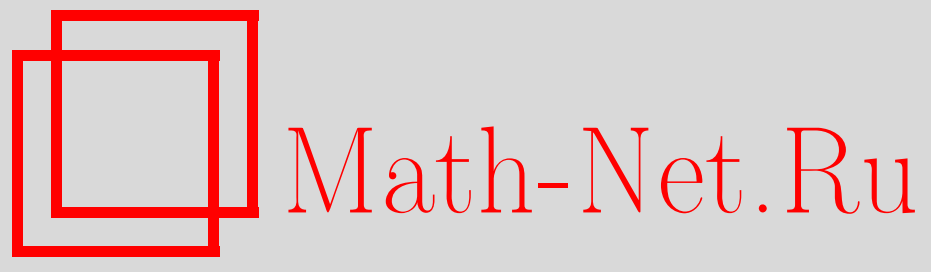

В. М. Имайкин, Солитонные асимптотики для систем типа "поле-частица", УМH, 2013, том 68, выпуск 2, 3390

DOI: https://doi.org/10.4213/rm9510

Использование Общероссийского математического портала Math-Net.Ru подразумевает, что вы прочитали и согласны с пользовательским соглашением http://www.mathnet.ru/rus/agreement

Параметры загрузки:

IP: 54.197 .217 .227

26 апреля 2023 г., 17:36:18

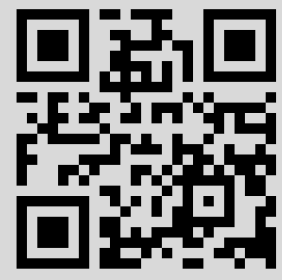




\section{Солитонные асимптотики для систем типа "поле-частица"}

\section{В. М. Имайкин}

Настоящий обзор посвящен недавнему математическому прогрессу в проблеме взаимодействия частицы с полем и охватывает цикл работ, выполненных с начала 2000-х годов по настоящее время. Рассматриваются три системы, описывающие взаимодействие поля с заряженной частицей: скалярное поле Клейна-Гордона или волновое поле, взаимодействующее с частицей, а также система Максвелла-Лоренца, описывающая заряженную частицу в поле Максвелла. В первых работах о долговременной сходимости к солитону в отсутствие внешнего потенциала (1990-е гг.) было впервые введено винеровское условие на плотность заряда частицы, которое оказалось существенным в отраженных в настоящем обзоре исследованиях по солитонным асимптотикам для решений с начальными данными, достаточно близкими к инвариантным солитонным многообразиям. Подход основан на эксплуатации гамильтоновой структуры систем и методе симплектической проекции Буслаева-Перельман.

Библиография: 49 названий.

Ключевые слова: нелинейная система типа "поле-частица", солитон, солитонное многообразие, симплектическая проекция, линеаризация на солитоне, модуляционные уравнения, убывание трансверсальной компоненты, винеровское условие.

DOI: $10.4213 / \mathrm{rm} 9510$

\section{СоДЕРЖАНИЕ}

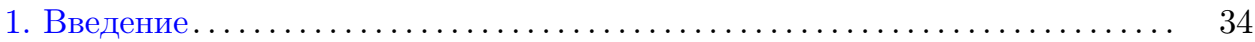

1.1. Поле, взаимодействующее с частицей ................. 34

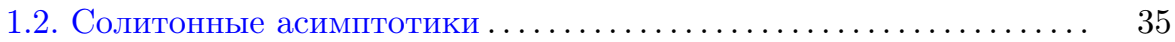

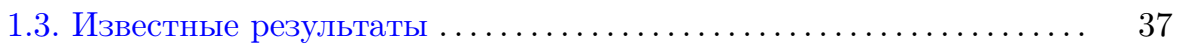

1.4. Теория рассеяния Буслаева-Перельман................ 37

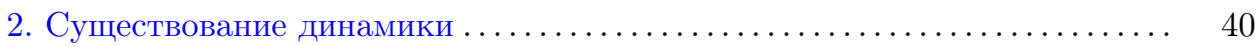

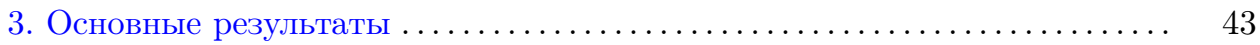

4. Симплектическая структура и симплектическая проекция .......... 46

5. Линеаризация на солитонном многообразии .................. 48

(C) В. М. ИМАЙКИН, 2013 
6. Симплектическое разложение динамики $\ldots \ldots \ldots \ldots \ldots \ldots \ldots \ldots \ldots \ldots \ldots \ldots$

6.1. Динамика вдоль солитонного многообразия: модуляционные

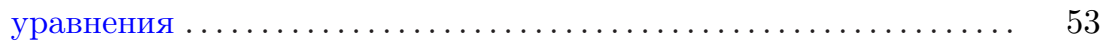

6.2. Убывание для трансверсальной динамики ............... 55

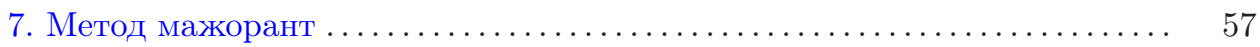

7.1. "Замораживание” трансверсальной динамики ............. 57

7.2. Интегральное неравенство ........................ 58

7.3. Симплектическая ортогональность ................... 59

7.4. Убывание трансверсальной компоненты ................ 61

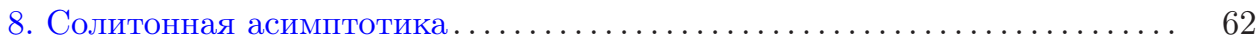

9. Убывание для линеаризованной динамики ................... 64

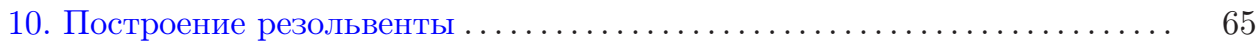

11. Свойства резольвенты . . . . . . . . . . . . . . . . . . . . . . 70

11.1. Аналитичность в правой полуплоскости ................. 70

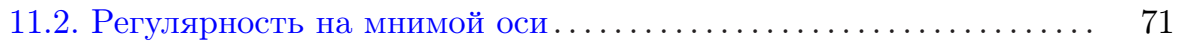

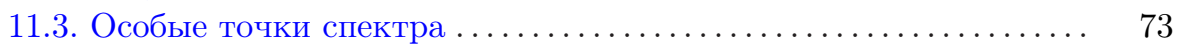

12. Временно́е убывание векторных компонент ................ 77

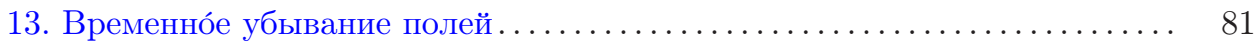

Приложение А. Вычисление симплектической формы ............. 84

Приложение В. Структура матрицы $M^{-1}(i \omega) \ldots \ldots \ldots \ldots \ldots \ldots \ldots \ldots . \ldots \ldots$

Список литературы ....................................... 88

\section{1. Введение}

Наш обзор посвящен недавнему математическому прогрессу в очень старой и принципиально важной области теоретической физики, а именно в проблеме взаимодействия частицы с полем. Уравнение движения заряженной частицы в электрическом поле было введено Лоренцем в 1895 г. (см. [1], [2]), хотя впервые оно было написано Максвеллом в одной из работ 1865 г. (см. [3]). С другой стороны, формулы для поля движущегося заряда были найдены Льенаром в 1898 г. и Вихертом в 1900 г. [4]. Таким образом, возникает проблема взаимодействия заряда с создаваемым им самим полем, т. е. проблема "самодействия" заряженной частицы.

Этот вопрос приобрел особенную остроту в связи с проблемой бесконечной массы и энергии точечного заряда, поставленной Абрагамом [5] в 1905 г. Для преодоления этой трудности Абрагам ввел понятие "размазанного" электрона.

Формулы Льенара-Вихерта наводят на мысль, что создаваемое ускоренно движущимся зарядом поле уносит энергию, в результате чего ускорение должно стремиться к нулю, как написано во всех учебниках электродинамики. Однако строго доказать это впервые удалось лишь сто лет спустя для модели Абрагама. Изложению этих работ и посвящен данный обзор.

1.1. Поле, взаимодействующее с частицей. В настоящей работе рассматриваются три системы, описывающие взаимодействие поля с заряженной частицей:

- поле Клейна-Гордона, взаимодействующее с частицей; 
- волновое поле, взаимодействующее с частицей;

- система Максвелла-Лоренца, описывающая заряженную частицу в поле Максвелла.

В работах [6], [7] рассмотрены аналогичные системы для полей Шрёдингера и Дирака.

I. Рассмотрим скалярное поле Клейна-Гордона $\psi(x)$ в $\mathbb{R}^{3}$, взаимодействующее с релятивистской частицей. Пусть $q, p$ - координаты и импульс частицы соответственно, тогда система имеет вид

$$
\begin{gathered}
\dot{\psi}(x, t)=\pi(x, t), \quad \dot{\pi}(x, t)=\Delta \psi(x, t)-m^{2} \psi(x, t)-\rho(x-q(t)), \\
\dot{q}(t)=\frac{p(t)}{\sqrt{1+p^{2}(t)}}, \quad \dot{p}(t)=-\langle\nabla \psi(x, t), \rho(x-q(t))\rangle,
\end{gathered}
$$

$x \in \mathbb{R}^{3}, t \in \mathbb{R}$, где $m>0$. Первые два уравнения для полей эквивалентны уравнению Клейна-Гордона с источником $\rho(x-q)$. Механическую массу частицы, а также скорость распространения волны мы считаем равными 1. Вид третьего и четвертого уравнений для частицы в (1.1) определяется выбором релятивистской кинетической энергии $\sqrt{1+p^{2}}$ в гамильтониане $(2.3)$, см. ниже.

II. В случае волнового поля система имеет вид (1.1) с $m=0$. Этот случай требует специального рассмотрения в связи со слабым пространственным убыванием солитонов волнового уравнения по сравнению с уравнением Клейна-Гордона.

III. В случае поля Максвелла система в гамильтоновых переменных записывается в виде

$$
\begin{aligned}
\dot{E}(x, t) & =-\Delta A(x, t)-\Pi(\rho(x-q(t)) v(t)), \quad \dot{A}(x, t)=-E(x, t), \\
\dot{q}(t) & =\frac{P(t)-\langle A(x, t), \rho(x-q(t))\rangle}{\left[1+(P(t)-\langle A(x, t), \rho(x-q(t))\rangle)^{2}\right]^{1 / 2}}=: v(t), \\
\dot{P}(t) & =\sum_{k=1}^{3} v_{k}(t)\left\langle\nabla A_{k}(x, t), \rho(x-q(t))\right\rangle,
\end{aligned}
$$

кроме того, налагаются условия трансверсальности

$$
\nabla \cdot E(x, t)=0, \quad \nabla \cdot A(x, t)=0 .
$$

Здесь П - проектор на пространство соленоидальных (бездивергентных) полей (см. подробности в [8], [9]). В представлении Фурье проектор имеет вид $\widehat{\Pi}(k) a=$ $a-\left(a \cdot k / k^{2}\right) k$.

Случай поля Максвелла идейно имеет много общего с волновым уравнением, однако алгебраическая структура задачи и соответствующие вычисления существенно усложняются.

1.2. Солитонные асимптотики. Система (1.1) трансляционно инвариантна и имеет солитонные решения вида

$$
Y_{a, v}(t)=\left(\psi_{v}(x-v t-a), \pi_{v}(x-v t-a), v t+a, p_{v}\right), \quad p_{v}=\frac{v}{\sqrt{1-v^{2}}},
$$


при всех $a, v \in \mathbb{R}^{3}$ с $|v|<1$ и всех $m \geqslant 0$ (см. (3.4), (3.5)). Аналогично, гамильтонова система Максвелла-Лоренца (1.2)-(1.5) имеет солитонные решения вида

$$
Y_{a, v}(t)=\left(E_{v}(x-v t-a), A_{v}(x-v t-a), v t+a, P_{v}\right), \quad P_{v}=\frac{v}{\sqrt{1-v^{2}}}+\left\langle\rho, A_{v}\right\rangle,
$$

при всех $a, v \in \mathbb{R}^{3}$ с $|v|<1$. Состояния $S_{a, v}:=Y_{a, v}(0)$ образуют гладкое солитонное подмногообразие размерности 6

$$
\mathscr{S}:=\left\{S_{a, v}: a, v \in \mathbb{R}^{3},|v|<1\right\}
$$

в гильбертовом фазовом пространстве.

В случае уравнения Клейна-Гордона мы требуем, чтобы все "моды” волнового поля взаимодействовали с частицей; это требование формализуется условием Винера

$$
\widehat{\rho}(k)=(2 \pi)^{-3 / 2} \int e^{i k x} \rho(x) d x \neq 0 \quad \forall k \in \mathbb{R}^{3} .
$$

Это условие является аналогом “золотого правила Ферми" [10]-[15]: член взаимодействия $\rho(x-q)$ не должен быть ортогонален собственным функциям $e^{i k x}$ непрерывного спектра линейной части уравнения. Как мы увидим далее, условие Винера (1.9) окажется очень существенным для нашего асимптотического анализа. Общие примеры функций взаимодействия $\rho$, удовлетворяющих $(2.8)$ и (1.9), построены в [16].

В случае волнового уравнения и уравнения Максвелла мы накладываем дополнительные условия равенства нулю первых четырех моментов плотности $\rho$, т. e.

$$
\int x^{\mu} \rho(x) d x=0, \quad|\mu| \leqslant 4 .
$$

Эти условия обеспечивают достаточно быстрое пространственное убывание солитонов. В терминах преобразования Фурье условия имеют следующий вид: $\widehat{\rho}(k) \neq 0$ при $k \neq 0$, но при $k=0$ функция $\widehat{\rho}(k)$ имеет нуль пятого порядка. Физический смысл условий при $\mu=0$ - электрическая нейтральность атома, которая обеспечивает его устойчивость. Такие плотности существуют: например, если $\rho_{1}$ удовлетворяет условиям (1.9), то, применив к ней достаточное число раз оператор Лапласа, получим функцию $\rho$, удовлетворяющую (1.10).

Нашим основным результатом для системы (1.1) является солитонная асимптотика при $t \rightarrow \pm \infty$ :

$$
(\psi(x, t), \pi(x, t)) \sim\left(\psi_{v_{ \pm}}\left(x-v_{ \pm} t-a_{ \pm}\right), \pi_{v_{ \pm}}\left(x-v_{ \pm} t-a_{ \pm}\right)\right)+W_{0}(t) \boldsymbol{\Psi}_{ \pm}
$$

при начальных данных, близких к солитонному многообразию $\mathscr{S}$. Здесь $W_{0}(t)-$ динамическая группа свободного уравнения, соответствующего $\rho=0, \boldsymbol{\Psi}_{ \pm}-$ соответствующие асимптотические состояния рассеяния, а остаток стремится к нулю в некоторой глобальной энергетической норме. 
В случае системы Максвелла-Лоренца асимптотика имеет вид

$(E(x, t), A(x, t)) \sim\left(E_{v_{ \pm}}\left(x-v_{ \pm} t-a_{ \pm}\right), A_{v_{ \pm}}\left(x-v_{ \pm} t-a_{ \pm}\right)\right)+W^{0}(t) \boldsymbol{\Psi}_{ \pm}, \quad t \rightarrow \pm \infty$.

Здесь $W^{0}(t)$ - динамическая группа свободного волнового уравнения в пространстве соленоидальных полей (т. е. уравнений (1.2) с $\rho=0$ и уравнений (1.5)), $\boldsymbol{\Psi}_{ \pm}-$соответствующие асимптотические поля рассеяния, а остаток стремится к нулю в глобальной энергетической норме.

Для траектории частицы мы во всех случаях доказываем, что

$$
\dot{q}(t) \rightarrow v_{ \pm}, \quad q(t) \sim v_{ \pm} t+a_{ \pm}, \quad t \rightarrow \pm \infty .
$$

Асимптотику (1.11) можно интерпретировать как столкновение входящего солитона с траекторией $v_{-} t+a_{-}$и входящей свободной волны $W_{0}(t) \boldsymbol{\Psi}_{-}$, которое приводит в результате к выходящему солитону с новой траекторией $v_{+} t+a_{+}$, а также новой выходящей свободной волне $W_{0}(t) \boldsymbol{\Psi}_{+}$.

1.3. Известные результаты. Первые результаты о долговременной сходимости к стационарным состояниям были получены для частицы во внешнем потенциале, взаимодействующей с волновым полем [16]. Затем была доказана сходимость к солитону в отсутствие внешнего потенциала [17], [18].

В этих работах было впервые введено винеровское условие на плотность заряда частицы, которое является аналогом "Золотого правила Ферми" (Fermi Golden Rule), и асимптотики были доказаны для всех решений конечной энергии. Эти результаты были обобщены на уравнения Максвелла-Лоренца для частицы в поле Максвелла [8], [19] в отсутствие внешнего потенциала.

Цикл работ [20]-[24] посвящен солитонным асимптотикам для случая слабого взаимодействия, когда плотность заряда частицы достаточно мала. Такие асимптотики доказаны для всех решений конечной энергии уравнений Клейна-Гордона [20], волнового [21] и Максвелла [22], [23], а также для уравнения Максвелла с вращающейся частицей [24]. Краткий обзор упомянутых работ можно найти в [9].

Последние исследования в этой области посвящены солитонным асимптотикам для решений с начальными данными, достаточно близкими к инвариантным солитонным многообразиям: в [25] - для уравнения Клейна-Гордона, в [26], [27] - для волнового уравнения, в [28] - для поля Максвелла. Именно эти результаты являются предметом настоящего обзора.

Наш подход, основанный на гамильтоновой структуре динамической системы и учитывающий симплектическую геометрию фазового пространства, развивает методы нелинейной теории рассеяния, инициированные в работах Зоффера и Вайнштейна [14], [15], [29]-[32] и развитые в работах Буслаева, Перельман и Сулем [10], [11], [33], [34], а также Куканья [12], [13].

1.4. Теория рассеяния Буслаева-Перельман. Прокомментируем основные трудности доказательства солитонных асимптотик (1.11)-(1.13). Методы работ [8], [17] основаны на винеровской тауберовой теореме и не дают возможности установить скорость сходимости в (1.11)-(1.13). Методы работ [8], 
[21], [22], [24] применимы только в случае достаточно малой функции взаимодействия $\rho(x)$ и не позволяют получить вторую асимптотику в (1.13).

Наш подход развивает общую стратегию, разработанную в [33]-[36], [14], [15], [32] для доказательства солитонных асимптотик в случае уравнений Шрёдингера и Клейна-Гордона с потенциалом. Эта стратегия впервые была предложена в работах [29]-[31], [37] в контексте $U(1)$-инвариантного уравнения Шрёдингера. Подход использует методы симплектической геометрии в гильбертовом пространстве для гамильтоновых систем, а также спектральную теорию несамосопряженных операторов.

Солитонные асимптотики означают асимптотическую устойчивость соответствующих солитонных многообразий $\mathscr{S}$ в фазовом пространстве. Такие инвариантные многообразия автоматически возникают для уравнений, инвариантных относительно некоторой группы Ли симметрий [38]-[40]. В частности, системы (1.1) и (1.2)-(1.5) инвариантны относительно сдвигов в $\mathbb{R}^{3}$. Асимптотическая устойчивость солитонного многообразия доказывается при помощи линеаризации динамики (2.1). Линеаризация осуществляется вдоль симплектически ортогональной проекции $S(t)$ решения на солитонное многообразие. Линеаризованное уравнение имеет вид

$$
\dot{X}(t)=A(t) X(t), \quad t \in \mathbb{R},
$$

где оператор $A(t)$ соответствует линеаризации на солитоне $S(t)$. Далее мы рассматриваем "замороженное" линеаризованное уравнение (1.14) с $A\left(t_{1}\right)$ вместо $A(t)$. Оператор $A\left(t_{1}\right)$ имеет собственные и присоединенные векторы, соответствующие нулевому собственному значению, и, соответственно, замороженное уравнение имеет растущие секулярные решения (6.13). Существование таких растущих решений препятствует прямому применению метода Ляпунова и является причиной неустойчивости нелинейной динамики вдоль многообразия $\mathscr{S}$. А именно, расстояние между солитонами (1.6) с близкими, но разными параметрами $v$ не остается малым, так как разность их координат $v t+a$ растет линейно по времени.

Ключевое наблюдение В. С. Буслаева с соавторами [33], [34], [10], [11] состоит в том, что при некоторых предположениях о спектре линеаризованной задачи (в нашем случае они заменяются винеровским условием) линеаризованное уравнение является устойчивым в трансверсальном симплектически ортогональном дополнении к касательному пространству $\mathscr{T}_{S}$. Это дополнение инвариантно относительно линеаризованной динамики, поскольку линеаризованная динамика также гамильтонова и сохраняет инвариантной симплектическую структуру.

Наше доказательство развивает методы работ [33]-[36]. Прокомментируем основные шаги.

I. Сначала строится симплектически ортогональная проекция $S(t)=\Pi Y(t)$ траектории $Y(t)$ на солитонное многообразие $\mathscr{S}$. Это значит, что $S(t) \in \mathscr{S}$ и дополнительный вектор $Z(t):=Y(t)-S(t)$ симплектически ортогонален к касательному пространству $\mathscr{T}_{S(t)} \mathscr{S}$ при каждом $t \in \mathbb{R}$ :

$$
Z(t) \nmid \mathscr{T}_{S(t)} \mathscr{S}, \quad t \in \mathbb{R} .
$$


Таким образом, возникает разложение $Y(t)=S(t)+Z(t)$, и мы линеаризуем динамику по трансверсальной компоненте $Z(t)$ вдоль траектории. Далее мы разделяем динамику на движение вдоль солитонного многообразия и движение в трансверсальных направлениях.

А. Движение вдоль $\mathscr{S}$ определяется солитонной проекцией $S(t)=S_{b(t), v(t)}$, где параметры $b(t), v(t)$ удовлетворяют модуляиионным уравнениям. А именно, в параметрах $\xi(t)=(c(t), v(t)), c(t):=b(t)-\int_{0}^{t} v(s) d s$, мы получаем

$$
\dot{\xi}(t)=N_{1}(\xi(t), Z(t)), \quad\left|N_{1}(\xi(t), Z(t))\right| \leqslant C\|Z(t)\|_{-\beta}^{2},
$$

где $\|\cdot\|_{-\beta}$ обозначает подходящую весовую норму Соболева.

ЗАмечАниЕ 1.1. Оценка в (1.16) означает, что для решения, лежащего вблизи солитонного многообразия, параметры симплектической проекции $c(t)$ и $v(t)$ изменяются "сверхмедленно", т. е. являются адиабатическими инвариантами (см. [41]).

В. С другой стороны, трансверсальная компонента $Z(t)$ удовлетворяет трансверсальному уравнению

$$
\dot{Z}(t)=A(t) Z(t)+N_{2}(S(t), Z(t)),
$$

где $A(t)=A_{c(t), v(t)}$ - линейный оператор, а $N_{2}(S(t), Z(t))$ - нелинейная часть второго порядка малости относительно $Z(t)$ :

$$
\left\|N_{2}(S(t), Z(t))\right\|_{\beta} \leqslant C\|Z(t)\|_{-\beta}^{2}
$$

здесь $\|\cdot\|_{\beta}$ определятся аналогично $\|\cdot\|_{-\beta}$. Заметим, что оценка (1.18) не является прямым следствием линеаризации, поскольку функция $S(t)$, вообще говоря, не есть стационарное решение уравнения (2.1). Основную роль в доказательстве (1.18) играют модуляционное уравнение и оценка (1.16).

II. Линеаризованная динамика (1.14) является неавтономной. Сначала мы фиксируем $t=t_{1}$ в $A(t)$ и рассматриваем соответствующее "замороженное" линейное автономное уравнение с $A\left(t_{1}\right)$ вместо $A(t)$. Мы доказываем убывание

$$
\|X(t)\|_{-\beta} \leqslant \frac{C\|X(0)\|_{\beta}}{(1+|t|)^{3 / 2}}, \quad t \in \mathbb{R},
$$

решений $X(t)$ замороженного уравнения для любого $X(0) \in \mathscr{Z}_{S_{1}}$, где $S_{1}:=$ $S\left(t_{1}\right)$, а $\mathscr{Z}_{S_{1}}$ - пространство векторов $X$, симплектически ортогональных к касательному пространству $\mathscr{T}_{S_{1}} \mathscr{S}$. Отметим, что убывание имеет место только для решений, симплектически ортогональных к касательному пространству. Основной причиной убывания является тот факт, что спектр генератора $A\left(t_{1}\right)$, суженного на пространство $\mathscr{Z}_{S_{1}}$, является чисто непрерывным.

Оценка (1.19) написана для случая уравнения Клейна-Гордона. В случае волнового уравнения и уравнения Максвелла показатели весов и степень убывания другие, см. ниже теорему 3.4 и оценку (6.19). То же самое относится к следующей оценке (1.20). 
III. Убывание (1.19) комбинируется с оценкой (1.16) в нелинейном уравнении (1.17). Это приводит к временно́му убыванию трансверсальной компоненты:

$$
\|Z(t)\|_{-\beta} \leqslant \frac{C\left(\|Z(0)\|_{\beta}\right)}{(1+|t|)^{3 / 2}}, \quad t \in \mathbb{R},
$$

если норма $\|Z(0)\|_{\beta}$ достаточно мала. Одна из основных трудностей при доказательстве убывания (1.20) - неавтономный характер линейной части (1.17). Мы выводим убывание из уравнения (1.17), записанного в "замороженной" форме

$$
\dot{Z}(t)=A\left(t_{1}\right) Z(t)+\left[A(t)-A\left(t_{1}\right)\right] Z(t)+N_{1}(S(t), Z(t)), \quad 0 \leqslant t<t_{1},
$$

с произвольно большим $t_{1}>0$. Для доказательства убывания (1.20) мы оцениваем разность операторов $A(t)-A\left(t_{1}\right)$ и применяем метод мажсорант.

Решающую роль в оценке разности $A(t)-A\left(t_{1}\right)$ играют модуляционные уравнения (1.16), которые означают, что параметры $c(t)$ и $v(t)$ меняются "сверхмедленно" и, соответственно, операторы $A(t)$ и $A_{1}(t)$ являются "сверхблизкими".

IV. Из убывания (1.20) выводятся солитонные асимптотики (1.11) и (1.13) при помощи известной техники теории рассеяния (метод Кука). Обратим внимание, что скорость сходимости оказывается разной в случае уравнения Клейна-Гордона $(m>0)$ и в случае волнового уравнения $(m=0)$ и уравнения Максвелла, что объясняется разной скоростью убывания для этих случаев в (1.20); последнее объясняется разной степенью пространственного убывания солитонов.

ЗАМЕчАНИЕ 1.2. Причиной асимптотической устойчивости солитонного многообразия $\mathscr{S}$ является излучение энергии в бесконечность, которое проявляется как локальное убывание энергии (1.20).

Стратегия [33]-[36] была также развита в работах [10]-[15], [32], [42]. Отметим, что все эти работы содержат априорные предположения о дискретном и непрерывном спектре линеаризованной задачи. Мы же получаем полную информацию о спектре линеаризованной задачи при условии Винера, не вводя априорных предположений о спектре.

Условие Винера используется при доказательстве убывания (1.19) только в одном месте: при доказательстве леммы 11.4. Остальные предположения о функции $\rho$ можно ослабить: сферическая симметрия не является необходимой и можно также допустить, что $\rho$ не является финитной, а принадлежит определенному пространству Соболева с весом.

\section{2. Существование динамики}

Запишем задачу Коши для систем (1.1) и (1.2)-(1.5) в виде

$$
\dot{Y}(t)=F(Y(t)), \quad t \in \mathbb{R}, \quad Y(0)=Y_{0},
$$

где $Y(t):=(\psi(x, t), \pi(x, t), q(t), p(t))$, или $Y(t):=(E(x, t), A(x, t), q(t), P(t))$, а все производные понимаются в смысле обобщенных функций. Система (1.1), 
как в случае уравнения Клейна-Гордона $(m>0)$, так и в случае волнового уравнения $(m=0)$ имеет гамильтонову форму

$$
\dot{Y}=J \mathscr{D} H(Y), \quad J=\left(\begin{array}{cccc}
0 & 1 & 0 & 0 \\
-1 & 0 & 0 & 0 \\
0 & 0 & 0 & E_{3} \\
0 & 0 & -E_{3} & 0
\end{array}\right),
$$

где $\mathscr{D}$ - производная $Ф$ реше, $E_{3}$ - единичная матрица размера $3 \times 3$. Для системы Клейна-Гордона (1.1) гамильтониан имеет вид

$$
\begin{gathered}
\mathscr{H}_{m}(\psi, \pi, q, p)=\frac{1}{2} \int\left(|\pi(x)|^{2}+|\nabla \psi(x)|^{2}+m^{2}|\psi(x)|^{2}\right) d x \\
+\int \psi(x) \rho(x-q) d x+\sqrt{1+p^{2}} .
\end{gathered}
$$

Для волновой системы гамильтониан равен $\mathscr{H}_{m}$ с массой $m=0$. Гамильтониан системы Максвелла-Лоренца имеет вид

$$
H(E, A, q, P)=\frac{1}{2} \int\left(|E|^{2}+|\nabla A|^{2}\right) d^{3} x+\left[1+\left(P-\int \rho(x-q) A(x) d^{3} x\right)^{2}\right]^{1 / 2},
$$

а сама система (1.2)-(1.5) - гамильтонову форму типа (2.2), с соответствующей модификацией.

Введем фазовое пространство для системы (1.1). Пусть $H^{0}=L^{2}$ - вещественное гильбертово пространство $L^{2}\left(\mathbb{R}^{3}\right)$ со скалярным произведением $\langle\cdot, \cdot\rangle$ и нормой $\|\cdot\|_{L^{2}}$, а $H^{1}$ - пространство Соболева $H^{1}=\left\{\psi \in L^{2}:|\nabla \psi| \in L^{2}\right\}$ с нормой $\|\psi\|_{H^{1}}=\|\nabla \psi\|_{L^{2}}+\|\psi\|_{L^{2}}$. Введем также пространства Соболева с весом $H_{\alpha}^{s}, s=0,1, \alpha \in \mathbb{R}$, с нормами $\|\psi\|_{s, \alpha}:=\left\|(1+|x|)^{\alpha} \psi\right\|_{H^{s}}$.

ОПРедЕЛЕНиЕ 2.1. і) Фазовое пространство $\mathscr{E}$ системы (1.1) при $m>0$ это вещественное гильбертово пространство $H^{1} \oplus L^{2} \oplus \mathbb{R}^{3} \oplus \mathbb{R}^{3}$ состояний $Y=$ $(\psi, \pi, q, p)$ и $\mathscr{F}$ - пространство $H^{1} \oplus L^{2}$ полей $F=(\psi, \pi)$ с конечными нормами

$$
\|Y\|_{\mathscr{E}}=\|\psi\|_{H^{1}}+\|\pi\|_{L^{2}}+|q|+|p|, \quad\|F\|_{\mathscr{F}}=\|\psi\|_{H^{1}}+\|\pi\|_{L^{2}}
$$

ii) $\mathscr{E}_{\alpha}-$ пространство $H_{\alpha}^{1} \oplus H_{\alpha}^{0} \oplus \mathbb{R}^{3} \oplus \mathbb{R}^{3}$ и $\mathscr{F}_{\alpha}-$ пространство $H_{\alpha}^{1} \oplus H_{\alpha}^{0}$ с нормами

$$
\|Y\|_{\alpha}=\|Y\|_{\mathscr{E}_{\alpha}}=\|\psi\|_{1, \alpha}+\|\pi\|_{0, \alpha}+|q|+|p|, \quad\|F\|_{\alpha}=\|F\|_{\mathscr{F}_{\alpha}}=\|\psi\|_{1, \alpha}+\|\pi\|_{0, \alpha} .
$$

Мы используем одни и те же обозначения для норм в пространстве $\mathscr{F} \alpha$ и в пространстве $\mathscr{E}_{\alpha}$, определенном в $(2.5)$. Из контекста всегда будет ясно, имеем ли мы дело только с полями, т. е. с элементами пространства $\mathscr{F}_{\alpha}$, или с полями и частицами, т. е. с элементами пространства $\mathscr{E}_{\alpha}$.

Для волнового уравнения, соответствующего $m=0$, мы вводим пространство $\dot{H}^{1}$ - пополнение вещественного пространства $C_{0}^{\infty}\left(\mathbb{R}^{3}\right)$ по норме $\|\nabla \psi(x)\|$, где | | - норма в $L^{2}\left(\mathbb{R}^{3}\right)$. В силу соответствующей теоремы вложения Соболева,

$$
\dot{H}^{1}=\left\{\psi(x) \in L^{6}\left(\mathbb{R}^{3}\right):|\nabla \psi(x)| \in L^{2}\right\} .
$$

Введем также пространство с весом $\dot{H}_{\alpha}^{1}$ с нормой $\|\psi\|_{\alpha}=\left\|(1+|x|)^{\alpha} \nabla \psi\right\|$. 
ОПРЕДЕЛЕниЕ 2.2. і) $\dot{\mathscr{E}}$ - гильбертово пространство $\dot{H}^{1} \oplus L^{2} \oplus \mathbb{R}^{3} \oplus \mathbb{R}^{3}$ и $\dot{\mathscr{F}}$ пространство $\dot{H}^{1} \oplus L^{2}$ полей $F=(\psi, \pi)$ с конечными нормами

$$
\|Y\|_{\mathscr{E}}=|\nabla \psi|+|\pi|+|q|+|p|, \quad Y=(\psi, \pi, q, p), \quad\|F\|_{\mathscr{F}}=|\nabla \psi|+|\pi| .
$$

ii) $\dot{\mathscr{E}}_{\alpha}-$ пространство $\dot{H}_{\alpha}^{1} \oplus L_{\alpha+1}^{2} \oplus \mathbb{R}^{3} \oplus \mathbb{R}^{3}$ и $\dot{\mathscr{F}}_{\alpha}-$ пространство $\dot{H}_{\alpha}^{1} \oplus L_{\alpha+1}^{2}$ с нормами

$$
\|Y\|_{\alpha}=\|Y\|_{\mathscr{E}_{\alpha}}=\|\psi\|_{\alpha}+|\pi|_{\alpha+1}+|q|+|p|, \quad\|F\|_{\alpha}=\|F\|_{\mathscr{F}_{\alpha}}=\|\psi\|_{\alpha}+|\pi|_{\alpha+1} .
$$

Заметим, что $\dot{H}^{1}$ не содержится в $L^{2}$, например, $\left|\psi_{v}\right|=\infty$ (см. ниже (3.5)), если не наложено условие (1.10). Однако $\dot{\mathscr{E}}$ является пространством состояний конечной энергии, т. е. $\mathscr{H}(Y)<\infty$ при $Y \in \dot{\mathscr{E}}$.

Наконец, введем фазовое пространство для системы (1.2)-(1.5). Положим $H^{0}=L^{2}\left(\mathbb{R}^{3}, \mathbb{R}^{3}\right), \dot{H}^{1}$ - замыкание пространства $C_{0}^{\infty}\left(\mathbb{R}^{3}, \mathbb{R}^{3}\right)$ по норме $\|A\|_{1}=$ $|\nabla A|=\|\nabla A\|_{L^{2}\left(\mathbb{R}^{3}, \mathbb{R}^{3}\right)}$. Обозначим $H_{s}^{0}, \dot{H}_{s}^{1}$ подпространства, образованные соленоидальными векторными полями, а именно замыкания в $H^{0}, \dot{H}^{1}$ соответственно векторных полей из $C_{0}^{\infty}$ с нулевой дивергенцией. Определим фазовое пространство $\mathscr{E}=H_{s}^{0} \oplus \dot{H}_{s}^{1} \oplus \mathbb{R}^{3} \oplus \mathbb{R}^{3}$ состояний $Y=(E, A, q, P)$ и пространство полей $\mathscr{F}=H_{s}^{0} \oplus \dot{H}_{s}^{1}$, с нормами

$$
\|Y\|_{\mathscr{E}}=|E|+\|A\|_{1}+|q|+|P|, \quad\|(E, A)\|_{\mathscr{F}}=|E|+\|A\|_{1} .
$$

Для формулировки основного результата необходимо ввести следующие соболевские пространства с весом. Пусть $H_{s, \alpha}^{0}, \dot{H}_{s, \alpha}^{1}-$ подпространства пространств $H_{s}^{0}$ (соответственно $\dot{H}_{s}^{1}$ ), состоящие из всех полей $E$ (соответственно $A)$ с конечными нормами $\|E\|_{0, \alpha}=\left|(1+|x|)^{\alpha} E\right|,\|A\|_{1, \alpha}=\left|(1+|x|)^{\alpha} \nabla A\right|$. Поскольку $\dot{H}_{s}^{1} \subset L^{6}\left(\mathbb{R}^{3}, \mathbb{R}^{3}\right)$, имеем $\dot{H}_{s}^{1} \subset H_{s, \alpha}^{0}, \alpha<-1$. Пусть $\mathscr{E}_{\alpha}=H_{s, \alpha+1}^{0} \oplus$ $\dot{H}_{s, \alpha}^{1} \oplus \mathbb{R}^{3} \oplus \mathbb{R}^{3}, \mathscr{F}_{\alpha}=H_{s, \alpha+1}^{0} \oplus \dot{H}_{s, \alpha}^{1}$ с нормами

$$
\|Y\|_{\alpha}=\left\|E_{s}\right\|_{0, \alpha+1}+\|A\|_{1, \alpha}+|q|+|P|, \quad\left\|\left(E_{s}, A\right)\right\|_{\alpha}=\left\|E_{s}\right\|_{0, \alpha+1}+\|A\|_{1, \alpha} .
$$

Мы накладываем следующие условия регулярности, убывания и симметрии на плотность распределения заряда: $\rho$ - вещественная функция, принадлежащая пространству Соболева $H^{2}\left(\mathbb{R}^{3}\right)$, с компактным носителем и сферически симметричная, т. е.

$$
\rho, \nabla \rho, \nabla \nabla \rho \in L^{2}\left(\mathbb{R}^{3}\right), \quad \rho(x)=0 \quad \text { при }|x| \geqslant R_{\rho}, \quad \rho(x)=\rho_{1}(|x|) .
$$

Сформулируем результаты о существовании и свойствах динамики. Для уравнений Клейна-Гордона $(m>0)$ и волнового $(m=0)$ справедливы следующие утверждения.

ПреДЛОЖЕНИЕ 2.3 [16], [20]. Пусть выполнены условия (2.8). Тогда:

i) для любого $Y_{0} \in \mathscr{E}\left(\right.$ соответственно $\left.Y_{0} \in \dot{\mathscr{E}}\right)$ задача Коши (2.1) имеет единственное решение $Y(t) \in C(\mathbb{R}, \mathscr{E})($ соответственно $Y(t) \in C(\mathbb{R}, \dot{\mathscr{E}}))$;

ii) для любого $t \in \mathbb{R}$ отображение $U(t): Y_{0} \mapsto Y(t)$ непрерывно на $\mathscr{E}(\dot{\mathscr{E}})$; 
iii) энергия сохраняется, m.е.

$$
\mathscr{H}_{m}(Y(t))=\mathscr{H}_{m}\left(Y_{0}\right) \quad\left(\mathscr{H}(Y(t))=\mathscr{H}\left(Y_{0}\right)\right), \quad t \in \mathbb{R}
$$

при этом скорость частицы ограничена:

$$
|\dot{q}(t)| \leqslant \bar{v}\left(Y_{0}\right)<1, \quad t \in \mathbb{R} .
$$

В случае уравнения Максвелла справедливы следующие утверждения.

ПреДЛОЖЕНИЕ 2.4 [8]. Предположим, что выполнены условия (2.8) и $Y_{0}=$ $\left(E_{0}, A_{0}, q_{0}, P_{0}\right) \in \mathscr{E}$. Тогда:

i) существует единственное решение $Y(t) \in C(\mathbb{R}, \mathscr{E})$ задачи Коши (1.2)-(1.5) с начальным условием $Y_{0}$;

ii) выполняется закон сохранения энергии $H(Y(t))=H\left(Y_{0}\right), t \in \mathbb{R}$;

iii) справедлива оченка скорости

$$
|\dot{q}(t)| \leqslant \bar{v}\left(Y_{0}\right)<1, \quad t \in \mathbb{R} .
$$

Доказательства предложений 2.3 и 2.4 при малых $|t|$ основаны на сведении к интегральному уравнению и применении метода сжимающих отображений. Глобальное решение для всех $t \in \mathbb{R}$ существует ввиду априорных энергетических оценок.

\section{3. Основные результаты}

Система (1.1) инвариантна относительно сдвигов и допускает солитонные решения

$$
Y_{a, v}(t)=\left(\psi_{v}(x-v t-a), \pi_{v}(x-v t-a), v t+a, p_{v}\right), \quad p_{v}=\frac{v}{\sqrt{1-v^{2}}},
$$

для любых $a, v \in \mathbb{R}^{3}$ c $|v|<1$. Вычислим солитоны для системы Клейна-Гордона. Подстановка в (1.1) приводит к следующим стационарным уравнениям:

$$
\begin{aligned}
-v \cdot \nabla \psi_{v}(y) & =\pi_{v}(y), & -v \cdot \nabla \pi_{v}(y) & =\Delta \psi_{v}(y)-m^{2} \psi_{v}(y)-\rho(y), \\
v & =\frac{p_{v}}{\sqrt{1+p_{v}^{2}}}, & 0 & =-\int \nabla \psi_{v}(y) \rho(y) d y .
\end{aligned}
$$

Из первых двух уравнений вытекает равенство

$$
\Lambda \psi_{v}(y):=\left[-\Delta+m^{2}+(v \cdot \nabla)^{2}\right] \psi_{v}(y)=-\rho(y), \quad y \in \mathbb{R}^{3} .
$$

Для $|v|<1$ оператор $\Lambda$ является изоморфизмом $H^{4}\left(\mathbb{R}^{3}\right) \rightarrow H^{2}\left(\mathbb{R}^{3}\right)$. Поэтому из $(2.8)$ следует, что $\psi_{v}(y)=-\Lambda^{-1} \rho(y) \in H^{4}\left(\mathbb{R}^{3}\right)$. Если фиксировано $v$ c $|v|<1$, то $p_{v}$ можно найти из третьего уравнения системы (3.2). Далее, функции $\rho$ и $\psi_{v}$ четные в силу (2.8). Поэтому функция $\nabla \psi_{v}$ нечетная и последнее уравнение системы (3.2) выполняется. В результате солитонное решение (3.1) существует и определяется однозначно для каждой пары $(a, v)$ 
c $|v|<1$. Явные формулы солитонов можно найти при помощи преобразования Фурье. В $x$-представлении солитоны задаются формулами

$$
\begin{aligned}
& \psi_{v}(x)=-\frac{\gamma}{4 \pi} \int \frac{e^{-m\left|\gamma(y-x)_{\|}+(y-x)_{\perp}\right|} \rho(y) d^{3} y}{\left|\gamma(y-x)_{\|}+(y-x)_{\perp}\right|} \\
& \pi_{v}(x)=-v \cdot \nabla \psi_{v}(x), \quad p_{v}=\gamma v=\frac{v}{\sqrt{1-v^{2}}} .
\end{aligned}
$$

Здесь $\gamma=1 / \sqrt{1-v^{2}}$ и $x=x_{\|}+x_{\perp}$, где $x_{\|} \| v$ и $x_{\perp} \perp v, x \in \mathbb{R}^{3}$. Обозначим $V:=\left\{v \in \mathbb{R}^{3}:|v|<1\right\}$.

В случае волнового уравнения получаем стационарные уравнения (3.2) с $m=0$. При $|v|<1$ они однозначно определяют функции $\psi_{v} \in \dot{H}^{1}\left(\mathbb{R}^{3}\right)$, $\pi_{v} \in L^{2}\left(\mathbb{R}^{3}\right)$. Солитоны имеют вид (3.4) с $m=0$ :

$$
\psi_{v}(x)=-\frac{1}{4 \pi} \int \frac{\rho(y) d^{3} y}{\left|\gamma(y-x)_{\|}+(y-x)_{\perp}\right|}, \quad \pi_{v}(x)=-v \cdot \nabla \psi_{v}(x), \quad p_{v}=\gamma v .
$$

В случае уравнения Максвелла солитоны имеют вид

$$
E(x, t)=E_{v}(x-v t), \quad A(x, t)=A_{v}(x-v t), \quad q(t)=v t, \quad P_{v}=p_{v}+\left\langle\rho, A_{v}\right\rangle,
$$

где $v \in \mathbb{R}^{3},|v|<1, E_{v}=\Pi E^{v}, A_{v}=\Pi A^{v}$, а $E^{v}, A^{v}, p_{v}$ задаются следующими формулами:

$$
\begin{array}{ccrl}
E^{v}(x)=-\nabla \phi_{v}(x)+v \cdot \nabla A^{v}(x), & A^{v}(x) & =v \phi_{v}(x) \\
\phi_{v}(x)=\int \frac{\gamma \rho(y) d^{3} y}{4 \pi\left|\gamma(y-x)_{\|}+(y-x)_{\perp}\right|}, & p_{v}=\frac{v}{\sqrt{1-v^{2}}} .
\end{array}
$$

Стационарные уравнения для $E_{v}, A_{v}, P_{v}$ имеют следующий вид:

$$
\begin{aligned}
& E_{v}(x)=v \cdot \nabla A_{v}(x), \quad v \cdot \nabla E_{v}(x)=\Delta A_{v}(x)+\Pi(\rho(x) v), \\
& v=\frac{P_{v}-\left\langle\rho, A_{v}\right\rangle}{\left[1+\left(P_{v}-\left\langle\rho, A_{v}\right\rangle\right)^{2}\right]^{1 / 2}}, \quad 0=\int \rho(x) v \cdot \nabla A_{v}(x) d^{3} x,
\end{aligned}
$$

ОПРЕДЕЛЕНИЕ 3.1. Солитонным состоянием называется состояние $S(\sigma):=$ $\left(\psi_{v}(x-b), \pi_{v}(x-b), b, p_{v}\right)$ для уравнений Клейна-Гордона и волнового, а также состояние $S(\sigma):=\left(E_{v}(x-b), A_{v}(x-b), b, P_{v}\right)$ для уравнения Максвелла, где $\sigma:=(b, v), b \in \mathbb{R}^{3}, v \in V$.

Солитонное решение допускает представление $S(\sigma(t))$, где $\sigma(t)=(b(t), v(t))=$ $(v t+a, v)$.

ОПРЕДЕЛЕНИЕ 3.2. Солитонное многообразие - это множество $\mathscr{S}:=\{S(\sigma)$ : $\left.\sigma \in \Sigma:=\mathbb{R}^{3} \times V\right\}$.

Основными результатами по асимптотической устойчивости солитонов являются следующие теоремы. Для поля Клейна-Гордона с $m>0$ имеет место следующая теорема. 
ТЕОрема 3.3. Пусть выполнены условия (2.8) и (1.9). Пусть $\beta>3 / 2$, а $Y(t)$ - решение задачи Коши (2.1) с начальными данными $Y_{0}$, достаточно близкими к солитонному многообразию:

$$
d_{0}:=\operatorname{dist}_{\mathscr{E}_{\beta}}\left(Y_{0}, \mathscr{S}\right) \ll 1 .
$$

Тогда выполняется следующая асимптотика при $t \rightarrow \pm \infty:$

$$
\begin{aligned}
& \dot{q}(t)=v_{ \pm}+\mathscr{O}\left(|t|^{-3 / 2}\right), \quad q(t)=v_{ \pm} t+a_{ \pm}+\mathscr{O}\left(|t|^{-1}\right) ; \\
& (\psi(x, t), \pi(x, t))=\left(\psi_{v_{ \pm}}\left(x-v_{ \pm} t-a_{ \pm}\right), \pi_{v_{ \pm}}\left(x-v_{ \pm} t-a_{ \pm}\right)\right) \\
& +W_{0}(t) \boldsymbol{\Psi}_{ \pm}+r_{ \pm}(x, t), \\
& \left\|r_{ \pm}(t)\right\|_{\mathscr{F}}=\mathscr{O}\left(|t|^{-1 / 2}\right) \text {. }
\end{aligned}
$$

Для волнового уравнения с $m=0$ имеет место следующая теорема.

Теорема 3.4. Пусть выполнены условия (2.8), винеровское условие (1.9), а также условие (1.10). Положим $\beta:=4+\delta, 0<\delta<1 / 2$. Рассмотрим решение $Y(t)$ задачи Коши (2.1) для системы (1.1) с начальным условием $Y_{0} \in$ $\mathscr{E}_{\beta}$, достаточно близким $\kappa$ солитонному многообразию:

$$
Y_{0}=S\left(\sigma_{0}\right)+Z_{0}, \quad d_{\beta}:=\left\|Z_{0}\right\|_{\beta} \ll 1 .
$$

Тогда выполняется следующая асимптотика при $t \rightarrow \pm \infty:$

$$
\begin{gathered}
\dot{q}(t)=v_{ \pm}+\mathscr{O}\left(|t|^{-1-\delta}\right), \quad q(t)=v_{ \pm} t+a_{ \pm}+\mathscr{O}\left(|t|^{-2 \delta}\right), \\
(\psi(x, t), \pi(x, t))=\left(\psi_{v_{ \pm}}\left(x-v_{ \pm} t-a_{ \pm}\right), \pi_{v_{ \pm}}\left(x-v_{ \pm} t-a_{ \pm}\right)\right) \\
+W_{0}(t) \boldsymbol{\Psi}_{ \pm}+r_{ \pm}(x, t), \\
\left\|r_{ \pm}(t)\right\| \mathscr{F}=\mathscr{O}\left(|t|^{-\delta}\right) .
\end{gathered}
$$

Для уравнения Максвелла имеет место следующая теорема.

Теорема 3.5. Пусть выполнены условие (2.8), винеровское условие (1.9), а также условие (1.10), пусть $\beta=4+\delta, 0<\delta<1 / 2$. Предположим, что $Y^{0} \in \mathscr{E}_{\beta}$ и что $Y^{0}$ достаточно близко $\kappa$ солитонному многообразию: $Y^{0}=$ $S\left(\sigma_{0}\right)+Z_{0}, d_{\beta}:=\left\|Z_{0}\right\|_{\beta} \ll 1$. Пусть $Y(t) \in C(\mathbb{R}, \mathscr{E})$ - решение задачи Коши (2.1) для системы (1.2)-(1.5). Тогда выполняется следующая асимптотика при $t \rightarrow \pm \infty$ :

$$
\begin{gathered}
\dot{q}(t)=v_{ \pm}+\mathscr{O}\left(|t|^{-1-\delta}\right), \quad q(t)=v_{ \pm} t+a_{ \pm}+\mathscr{O}\left(|t|^{-2 \delta}\right) \\
(E(x, t), A(x, t))=\left(E_{v_{ \pm}}\left(x-v_{ \pm} t-a_{ \pm}\right), A_{v_{ \pm}}\left(x-v_{ \pm} t-a_{ \pm}\right)\right) \\
+W^{0}(t) \boldsymbol{\Psi}_{ \pm}+r_{ \pm}(x, t) \\
\left\|r_{ \pm}(t)\right\|_{\mathscr{F}}=\mathscr{O}\left(|t|^{-\delta}\right) .
\end{gathered}
$$

Все указанные в теоремах асимптотики достаточно доказать при $t \rightarrow+\infty$, поскольку системы (1.1) и (1.2)-(1.5) обратимы по времени.

Ниже мы даем подробное изложение в основном для уравнения Клейна-Гордона, а затем указываем соответствующие изменения для волнового уравнения и уравнения Максвелла. 


\section{4. Симплектическая структура и симплектическая проекция}

Отождествим касательное пространство к $\mathscr{E}$ в каждой точке с самим $\mathscr{E}$. Рассмотрим симплектическую форму $\Omega$, заданную на $\mathscr{E}$ формулой $\Omega=\int d \psi(x) \wedge$ $d \pi(x) d x+d q \wedge d p$, т. е.

$\Omega\left(Y_{1}, Y_{2}\right)=\left\langle Y_{1}, J Y_{2}\right\rangle, \quad Y_{1}, Y_{2} \in \mathscr{E}, \quad\left\langle Y_{1}, Y_{2}\right\rangle:=\left\langle\psi_{1}, \psi_{2}\right\rangle+\left\langle\pi_{1}, \pi_{2}\right\rangle+q_{1} q_{2}+p_{1} p_{2}$,

$\left\langle\psi_{1}, \psi_{2}\right\rangle=\int \psi_{1}(x) \psi_{2}(x) d x$ и т. д. Очевидно, что форма $\Omega$ является невырожденной, т. е. если $\Omega\left(Y_{1}, Y_{2}\right)=0$ для любого $Y_{2} \in \mathscr{E}$, то $Y_{1}=0$.

ОПРеДЕЛЕНИЕ 4.1. і) $Y_{1} \nmid Y_{2}$ означает, что $Y_{1} \in \mathscr{E}, Y_{2} \in \mathscr{E}$ и $Y_{1}$ симплектически ортогонален $Y_{2}$, т. е. $\Omega\left(Y_{1}, Y_{2}\right)=0$.

ii) Оператор проектирования $\mathbf{P}: \mathscr{E} \rightarrow \mathscr{E}$ называется симплектически ортогональным, если $Y_{1} \nmid Y_{2}$ для $Y_{1} \in \operatorname{Ker} \mathbf{P}$ и $Y_{2} \in \operatorname{Im} \mathbf{P}$.

В случае волнового уравнения все определения сохраняются с заменой пространства $\mathscr{E}$ на $\dot{\mathscr{E}}$.

В случае уравнения Максвелла-Лоренца симплектическая форма $\Omega$ определена на $\mathscr{E}$ формулой

$$
\begin{aligned}
\Omega= & \int d E(x) \wedge d A(x) d x+d q \wedge d P, \\
& \quad \text { т. е. } \Omega\left(Y_{1}, Y_{2}\right)=\int\left(E_{1} \cdot A_{2}-E_{2} \cdot A_{1}\right) d x+q_{1} \cdot P_{2}-q_{2} \cdot P_{1}
\end{aligned}
$$

для $Y_{k}=\left(E_{k}, A_{k}, q_{k}, P_{k}\right) \in \mathscr{E}, k=1,2$, если интеграл сходится.

Рассмотрим касательное пространство $\mathscr{T}_{S(\sigma)} \mathscr{S}$ к многообразию $\mathscr{S}$ в точке $S(\sigma)$. Векторы $\tau_{j}:=\partial_{\sigma_{j}} S(\sigma)$, где $\partial_{\sigma_{j}}:=\partial_{b_{j}}$ и $\partial_{\sigma_{j+3}}:=\partial_{v_{j}}, j=1,2,3$, образуют базис в $\mathscr{T}_{\sigma} \mathscr{S}$. Подробнее,

$$
\begin{gathered}
\tau_{j}=\tau_{j}(v):=\partial_{b_{j}} S(\sigma)=\left(-\partial_{j} \psi_{v}(y),-\partial_{j} \pi_{v}(y), e_{j}, 0\right), \\
\tau_{j+3}=\tau_{j+3}(v):=\partial_{v_{j}} S(\sigma)=\left(\partial_{v_{j}} \psi_{v}(y), \quad \partial_{v_{j}} \pi_{v}(y), 0, \partial_{v_{j}} p_{v}\right), \quad j=1,2,3,
\end{gathered}
$$

где $y:=x-b-$ "подвижная система координат", $e_{1}=(1,0,0)$ и т. д. Подчеркнем, что функции $\tau_{j}$ всегда будут рассматриваться как функции от $y$, а не от $x$.

Из формул (3.4) и условий (2.8) следует, что

$$
\tau_{j}(v) \in \mathscr{E}_{\alpha}, \quad v \in V, \quad j=1, \ldots, 6, \quad \forall \alpha \in \mathbb{R} .
$$

ЛЕмма 4.2. Матрица с матричными элементами $\Omega\left(\tau_{l}(v), \tau_{j}(v)\right)$ невырождена для любого $v \in V$.

ДокАЗАТЕЛЬство. Матричные элементы вычислены в приложении А. В результате матрица $\Omega\left(\tau_{l}, \tau_{j}\right)$ имеет вид

$$
\Omega(v):=\left(\Omega\left(\tau_{l}, \tau_{j}\right)\right)_{l, j=1, \ldots, 6}=\left(\begin{array}{cc}
0 & \Omega^{+}(v) \\
-\Omega^{+}(v) & 0
\end{array}\right),
$$


где $(3 \times 3)$-матрица $\Omega^{+}(v)$ равна $\mathscr{K}+\left(1-v^{2}\right)^{-1 / 2} E+\left(1-v^{2}\right)^{-3 / 2} v \otimes v$. Здесь $\mathscr{K}$ - симметрическая $(3 \times 3)$-матрица с элементами

$$
\mathscr{K}_{i j}=\int d k\left|\widehat{\psi}_{v}(k)\right|^{2} k_{i} k_{j} \frac{k^{2}+m^{2}+3(k v)^{2}}{k^{2}+m^{2}-(k v)^{2}}=\int d k|\widehat{\rho}(k)|^{2} k_{i} k_{j} \frac{k^{2}+m^{2}+3(k v)^{2}}{\left(k^{2}+m^{2}-(k v)^{2}\right)^{3}} .
$$

Матрица $\mathscr{K}$ является результатом интегрирования симметричной неотрицательно определенной матрицы $k \otimes k=\left(k_{i} k_{j}\right)$ с положительным весом. Поэтому матрица $\mathscr{K}$ также неотрицательно определена. Поскольку единичная матрица $E$ положительно определена, а матрица $v \otimes v$ неотрицательно определена, матрица $\Omega^{+}(v)$ симметрична и положительно определена, а значит, невырождена. Тогда и матрица $\Omega\left(\tau_{l}, \tau_{j}\right)$ также невырождена. Лемма доказана.

Покажем, что в малой окрестности солитонного многообразия $\mathscr{S}$ определена "симплектическая ортогональная проекция" на $\mathscr{S}$. Введем преобразования сдвига $T_{a}:(\psi(\cdot), \pi(\cdot), q, p) \mapsto(\psi(\cdot-a), \pi(\cdot-a), q+a, p), a \in \mathbb{R}^{3}$. Заметим, что многообразие $\mathscr{S}$ инвариантно относительно этих преобразований. Обозначим $v(p):=p / \sqrt{1+p^{2}}$ для $p \in \mathbb{R}^{3}$.

ОПРЕДЕЛЕНИЕ 4.3. і) Для любого $\alpha \in \mathbb{R}$ и $\bar{v}<1$ обозначим $\mathscr{E}_{\alpha}(\bar{v})=\{Y=$ $\left.(\psi, \pi, q, p) \in \mathscr{E}_{\alpha}:|v(p)| \leqslant \bar{v}\right\}$. Положим $\mathscr{E}(\bar{v}):=\mathscr{E}_{0}(\bar{v})$.

ii) Для любого $\widetilde{v}<1$ обозначим $\Sigma(\widetilde{v})=\left\{\sigma=(b, v): b \in \mathbb{R}^{3},|v| \leqslant \widetilde{v}\right\}$.

Лемма 4.4 [25]. Пусть выполнено условие (2.8), $\alpha \in \mathbb{R} u \bar{v}<1$. Тогда:

i) существуют окрестность $\mathscr{O}_{\alpha}(\mathscr{S})$ многообразия $\mathscr{S}$ в $\mathscr{E}_{\alpha}$ и отображение $\Pi: \mathscr{O}_{\alpha}(\mathscr{S}) \rightarrow \mathscr{S}$ такие, что $\Pi$ равномерно непрерьвно на $\mathscr{O}_{\alpha}(\mathscr{S}) \cap \mathscr{E}_{\alpha}(\bar{v})$ в метрике пространства $\mathscr{E}_{\alpha}$,

$$
\Pi Y=Y \text { при } Y \in \mathscr{S}, \quad \text { u } Y-S \nmid \mathscr{T}_{S} \mathscr{S}, \text { дде } S=\Pi Y ;
$$

ii) окрестность $\mathscr{O}_{\alpha}(\mathscr{S})$ инвариантна относительно сдвигов $T_{a}, u$

$$
\Pi T_{a} Y=T_{a} \Pi Y \quad \text { nрu } Y \in \mathscr{O}_{\alpha}(\mathscr{S}) u a \in \mathbb{R}^{3}
$$

iii) для любого $\bar{v}<1$ существует $\widetilde{v}<1$ такое, ито $\Pi Y=S(\sigma)$, где $\sigma \in \Sigma(\widetilde{v})$, для $Y \in \mathscr{O}_{\alpha}(\mathscr{S}) \cap \mathscr{E}_{\alpha}(\bar{v})$;

iv) для любого $\widetilde{v}<1$ существует $r_{\alpha}(\widetilde{v})>0$ такое, ито $S(\sigma)+Z \in \mathscr{O}_{\alpha}(\mathscr{S})$, если $\sigma \in \Sigma(\widetilde{v}) u\|Z\|_{\alpha}<r_{\alpha}(\widetilde{v})$.

ДокАзАтЕЛьство. Требуется найти такое $\sigma=\sigma(Y)$, что $S(\sigma)=\Pi Y$ и

$$
\Omega\left(Y-S(\sigma), \partial_{\sigma_{j}} S(\sigma)\right)=0, \quad j=1, \ldots, 6 .
$$

Зафиксируем произвольное $\sigma^{0} \in \Sigma$ и заметим, что в систему (4.9) входят только 6 гладких скалярных функций от $Y$. Тогда для $Y$, близких к $S\left(\sigma^{0}\right)$, существование $\sigma$ будет следовать из стандартной конечномерной теоремы о неявной функции, если мы покажем, что матрица Якоби размера $6 \times 6$ с элементами $M_{l j}(Y)=\partial_{\sigma_{l}} \Omega\left(Y-S\left(\sigma^{0}\right), \partial_{\sigma_{j}} S\left(\sigma^{0}\right)\right)$ является невырожденной в точке $Y=$ $S\left(\sigma^{0}\right)$. Заметим, что все производные существуют в силу (4.4). Невырожденность вытекает из леммы 4.2, поскольку $M_{l j}\left(S\left(\sigma^{0}\right)\right)=-\Omega\left(\partial_{\sigma_{l}} S\left(\sigma^{0}\right), \partial_{\sigma_{j}} S\left(\sigma^{0}\right)\right)$. 
Тогда существует окрестность $\mathscr{O}_{\alpha}\left(S\left(\sigma^{0}\right)\right)$ точки $S\left(\sigma^{0}\right)$, где П определено и удовлетворяет (4.7), то же самое выполнено в объединении $\mathscr{O}_{\alpha}^{\prime}(\mathscr{S})=\bigcup_{\sigma^{0} \in \Sigma} \mathscr{O}_{\alpha}\left(S\left(\sigma^{0}\right)\right)$. Тождество (4.8) выполняется при $Y, T_{a} Y \in \mathscr{O}_{\alpha}^{\prime}(\mathscr{S})$, поскольку форма $\Omega$ и многообразие $\mathscr{S}$ инвариантны относительно сдвигов. Остается разнести окрестность $\mathscr{O}_{\alpha}^{\prime}(\mathscr{S})$ при помощи сдвигов: положим $\mathscr{O}_{\alpha}(\mathscr{S})=\bigcup_{b \in \mathbb{R}^{3}} T_{b} \mathscr{O}_{\alpha}^{\prime}(\mathscr{S})$. Тогда, очевидно, утверждение ii) выполнено. Утверждения iii) и iv), а также равномерная непрерывность утверждении і) следуют из инвариантности относительно сдвигов и соображений компактности. Лемма доказана.

Мы называем П симплектической ортогональной проекцией на $\mathscr{S}$.

Для волнового уравнения из формул (3.5) и условий (2.8), (1.10) следует, что если $|v|<1$, то $\psi_{v}(y) \sim|y|^{-6}, \pi_{v}(y) \sim|y|^{-7}$ при $|y| \rightarrow \infty$, а значит,

$$
\psi_{v} \in H_{\alpha}^{1}, \quad \alpha<\frac{9}{2} ; \quad \pi_{v} \in L_{\alpha}^{2}, \quad \alpha<\frac{11}{2} .
$$

Лемма 4.2 остается верной; при вычислении матричных элементов в приложении А при $m=0$ интегралы сходятся в силу условия (1.10).

Для уравнения Максвелла в силу (3.6) и условия (1.10) получаем, что $A_{v}(y)=$ $\mathscr{O}\left(|y|^{-6}\right), E_{v}(y)=\mathscr{O}\left(|y|^{-7}\right),|y| \rightarrow \infty$. Значит, $E_{v} \in H_{s, \alpha}^{0}$ при $\alpha<11 / 2$ и $A_{v} \in \dot{H}_{s, \alpha}^{1}$ при $\alpha<9 / 2$. Таким образом, и для волнового уравнения, и для уравнения Максвелла солитонные состояния удовлетворяют условию

$$
S(\sigma) \in \mathscr{E}_{\alpha} \quad \text { при } \alpha<\frac{9}{2} .
$$

Базис касательного пространства $\mathscr{T}_{S(\sigma)} \mathscr{S}$ к многообразию $\mathscr{S}$ в точке $S(\sigma)$ состоит из векторов

$$
\begin{aligned}
\tau_{j}=\tau_{j}(v) & :=\partial_{b_{j}} S(\sigma)=\left(-\partial_{j} E_{v}(y),-\partial_{j} A_{v}(y), e_{j}, 0\right), \\
\tau_{j+3}=\tau_{j+3}(v) & :=\partial_{v_{j}} S(\sigma)=\left(\partial_{v_{j}} E_{v}(y), \partial_{v_{j}} A_{v}(y), 0, \partial_{v_{j}} P_{v}\right), \quad j=1,2,3 .
\end{aligned}
$$

В случае волнового уравнения и уравнения Максвелла лемма 4.4 остается верной с $\alpha>-9 / 2$. Это связано с тем, что должна быть определена форма $\Omega\left(Y, \partial_{\sigma_{j}} S(\sigma)\right)$. Ввиду условий (4.10) нетрудно показать, что эта форма определена при $Y \in \dot{\mathscr{E}}_{\alpha}$, где $\alpha>-9 / 2$.

СлеДСТВИЕ 4.5 [25]. Из условия (3.8) следует, что $Y_{0}=S+Z_{0}$, әде $S=$ $S\left(\sigma_{0}\right)=\Pi Y_{0} u$

$$
\left\|Z_{0}\right\|_{\beta} \ll 1 \text {. }
$$

Для волнового уравнения и уравнения Максвелла следствие 4.5 также справедливо с показателем $\beta=4+\delta$ в (4.13), взятом из формулировок теорем $3.4,3.5$.

\section{5. Линеаризация на солитонном многообразии}

Рассмотрим решение системы (1.1)) и разложим его в сумму

$$
Y(t)=S(\sigma(t))+Z(t)
$$


где $\sigma(t)=(b(t), v(t)) \in \Sigma-$ произвольная гладкая функция от $t \in \mathbb{R}$. Подробнее, обозначим $Y=(\psi, \pi, q, p)$ и $Z=(\Psi, \Pi, Q, P)$. Тогда (5.1)) означает, что

$$
\begin{array}{ll}
\psi(x, t)=\psi_{v(t)}(x-b(t))+\Psi(x-b(t), t), & q(t)=b(t)+Q(t), \\
\pi(x, t)=\pi_{v(t)}(x-b(t))+\Pi(x-b(t), t), & p(t)=p_{v(t)}+P(t) .
\end{array}
$$

Подставим (5.2) в (1.1) и линеаризуем формально уравнения по $Z$. Положим $y=x-b(t)$, т. е. перейдем в "подвижную систему координат"; из (5.2) и (1.1) получим

$$
\begin{aligned}
\dot{\psi} & =\dot{v} \cdot \nabla_{v} \psi_{v}(y)-\dot{b} \cdot \nabla \psi_{v}(y)+\dot{\Psi}(y, t)-\dot{b} \cdot \nabla \Psi(y, t)=\pi_{v}(y)+\Pi(y, t), \\
\dot{\pi} & =\dot{v} \cdot \nabla_{v} \pi_{v}(y)-\dot{b} \cdot \nabla \pi_{v}(y)+\dot{\Pi}(y, t)-\dot{b} \cdot \nabla \Pi(y, t) \\
& =\Delta \psi_{v}(y)-m^{2} \psi_{v}(y)+\Delta \Psi(y, t)-m^{2} \Psi(y, t)-\rho(y-Q), \\
\dot{q} & =\dot{b}+\dot{Q}=\frac{p_{v}+P}{\sqrt{1+\left(p_{v}+P\right)^{2}}}, \\
\dot{p} & =\dot{v} \cdot \nabla_{v} p_{v}+\dot{P}=-\left\langle\nabla\left(\psi_{v}(y)+\Psi(y, t)\right), \rho(y-Q)\right\rangle .
\end{aligned}
$$

Эти уравнения приводятся к виду

$$
\dot{Z}(t)=A(t) Z(t)+T(t)+N(t), \quad t \in \mathbb{R} .
$$

Здесь оператор $A(t)=A_{v, w}$ зависит от двух параметров $v=v(t)$ и $w=\dot{b}(t)$ и имеет вид

$$
A_{v, w}\left(\begin{array}{c}
\Psi \\
\Pi \\
Q \\
P
\end{array}\right):=\left(\begin{array}{cccc}
w \cdot \nabla & 1 & 0 & 0 \\
\Delta-m^{2} & w \cdot \nabla & \nabla \rho \cdot & 0 \\
0 & 0 & 0 & B_{v} \\
\langle\cdot, \nabla \rho\rangle & 0 & \left\langle\nabla \psi_{v}, \cdot \nabla \rho\right\rangle & 0
\end{array}\right)\left(\begin{array}{c}
\Psi \\
\Pi \\
Q \\
P
\end{array}\right),
$$

где $B_{v}=\nu(E-v \otimes v), \nu=\sqrt{1-v^{2}}$. Далее,

$$
T(t)=T_{v, w}=\left(\begin{array}{c}
(w-v) \cdot \nabla \psi_{v}-\dot{v} \cdot \nabla_{v} \psi_{v} \\
(w-v) \cdot \nabla \pi_{v}-\dot{v} \cdot \nabla_{v} \pi_{v} \\
v-w \\
-\dot{v} \cdot \nabla_{v} p_{v}
\end{array}\right), \quad N(t)=N(\sigma(t), Z(t)),
$$

где $v=v(t), w=w(t), \sigma(t)=(b(t), v(t))$ и

$$
\|N(\sigma, Z)\|_{\beta} \leqslant C(\widetilde{v}, \bar{Q})\|Z\|_{-\beta}^{2}
$$

равномерно по $\sigma \in \Sigma(\widetilde{v})$ и $\|Z\|_{-\beta} \leqslant r_{-\beta}(\widetilde{v})$ для любого фиксированного $\widetilde{v}<1$. Оценка (5.6) выполнена при любом $\beta \in \mathbb{R}$. Ниже мы используем ее при фиксированном $\beta>3 / 2$, введенном в теореме 3.3 , в случае Клейна-Гордона и при введенном в теореме $3.4 \beta=4+\delta, 0<\delta<1 / 2$, в случае волнового уравнения (для уравнения Максвелла мы используем оценку (5.12) с тем же $\beta$, что и для волнового уравнения). 
ЗАмечАнИЕ 5.1. і) Член $A(t) Z(t)$ в правой части уравнения (5.3) линеен по $Z(t)$, а $N(t)$ - член более высокого порядка по $Z(t)$. Если проекция $S(\sigma(t))$ не является солитонным решением, то $T(t)$ - член нулевого порядка, который не обращается в нуль при $Z(t)=0$.

ii) Из формул (4.3) и (5.5) следует, что

$$
T(t)=-\sum_{l=1}^{3}\left[(w-v)_{l} \tau_{l}+\dot{v}_{l} \tau_{l+3}\right],
$$

поэтому $T(t) \in \mathscr{T}_{S(\sigma(t))} \mathscr{S}, t \in \mathbb{R}$.

Для волнового уравнения вся описанная выше процедура линеаризации сохраняется с подстановкой $m=0$.

Для уравнения Максвелла разложим решение системы (1.2)-(1.5) в сумму $Y(t)=S(\sigma(t))+Z(t)$, где $\sigma(t)=(b(t), v(t)) \in \mathbb{R}^{3} \times V$ - произвольная гладкая траектория. Подробнее, обозначим $Y=(E, A, q, P)$ и $Z=(e, a, r, \pi)$. Тогда

$$
\begin{aligned}
E(x, t) & =E_{v(t)}(x-b(t))+e(x-b(t), t), & q(t) & =b(t)+r(t), \\
A(x, t) & =A_{v(t)}(x-b(t))+a(x-b(t), t), & P(t) & =P_{v(t)}+\pi(t) .
\end{aligned}
$$

Подставим (5.8)) в (1.2)-(1.5) и линеаризуем полученные уравнения по $Z$. Пусть $y=x-b(t)$. Из (5.8) и (1.2)-(1.4) получаем:

$$
\begin{aligned}
\dot{E} & =\dot{v} \cdot \nabla_{v} E_{v(t)}(y)-\dot{b} \cdot \nabla E_{v(t)}(y)+\dot{e}(y, t)-\dot{b} \cdot \nabla e(y, t) \\
& =-\Delta\left(A_{v}(y)+a(y, t)\right)-\Pi(\rho(y-r) \dot{q}), \\
\dot{A} & =\dot{v} \cdot \nabla_{v} A_{v(t)}(y)-\dot{b} \cdot \nabla A_{v(t)}(y)+\dot{a}(y, t)-\dot{b} \cdot \nabla a(y, t)=-E_{v(t)}(y)-e(y, t), \\
\dot{q} & =\dot{b}+\dot{r}=\frac{P_{v(t)}+\pi-\left\langle\rho(y-r), A_{v(t)}(y)+a(y, t)\right\rangle}{\left(1+\left(P_{v(t)}+\pi-\left\langle\rho(y-r), A_{v(t)}(y)+a(y, t)\right\rangle\right)^{2}\right)^{1 / 2}}, \\
\dot{P} & =\dot{v} \cdot \nabla_{v} P_{v(t)}+\dot{\pi}=\left\langle\rho(y-r), \nabla\left(\dot{q} \cdot\left(A_{v(t)}(y)+a(y, t)\right)\right)\right\rangle .
\end{aligned}
$$

Эти уравнения приводятся к виду

$$
\dot{Z}(t)=A(t) Z(t)+T(t)+N(t), \quad t \in \mathbb{R} .
$$

Оператор $A(t)=A_{v, w}$ имеет вид

$$
A_{v, w}\left(\begin{array}{c}
e \\
a \\
r \\
\pi
\end{array}\right):=\left(\begin{array}{cccc}
w \cdot \nabla & -\Delta+\Pi\left(\rho B_{v}\langle\rho, \cdot\rangle\right) & \Pi(\cdot \nabla \rho v) & -\Pi\left(\rho B_{v} \cdot\right) \\
-1 & w \cdot \nabla & 0 & 0 \\
0 & -B_{v}\langle\rho, \cdot\rangle & 0 & B_{v} \\
0 & \langle\rho, \nabla(v \cdot)\rangle & -\left\langle\cdot \nabla \rho, \nabla\left(v \cdot A_{v}\right)\right\rangle & 0
\end{array}\right)\left(\begin{array}{c}
e \\
a \\
r \\
\pi
\end{array}\right) .
$$

Далее,

$$
T(t)=T_{v, w}=\left(\begin{array}{c}
(w-v) \cdot \nabla E_{v}-\dot{v} \cdot \nabla_{v} E_{v} \\
(w-v) \cdot \nabla A_{v}-\dot{v} \cdot \nabla_{v} A_{v} \\
v-w \\
-\dot{v} \cdot \nabla_{v} P_{v}
\end{array}\right), \quad N(t)=N(\sigma(t), Z(t)),
$$


$v=v(t), w=w(t), \sigma=\sigma(t)=(b(t), v(t))$ и

$$
\|N(\sigma, Z)\|_{\beta} \leqslant C(\widetilde{v}, \bar{Q})\|Z\|_{-\beta}^{2}
$$

равномерно по $\sigma$ с $|v|<\widetilde{v}$ и $\|Z\|_{-\beta} \leqslant r_{-\beta}(\widetilde{v})$ для любого фиксированного $\widetilde{v}<1$.

Изучим свойства линеаризованного уравнения с произвольными $v \in V$ и $w \in \mathbb{R}^{3}$ :

$$
\dot{X}(t)=A_{v, w} X(t), \quad t \in \mathbb{R} .
$$

Лемма 5.2 [25]. і) Для любых $v \in V u w \in \mathbb{R}^{3}$ уравнение (5.13) имеет гамильтонову борму

$$
\dot{X}(t)=J D \mathscr{H}_{v, w}(X(t)), \quad t \in \mathbb{R},
$$

где D $\mathscr{H}_{v, w}$ - производная Фреше гамильтониана

$$
\begin{aligned}
\mathscr{H}_{v, w}(X)=\frac{1}{2} \int\left[|\Pi|^{2}+|\nabla \Psi|^{2}+m^{2}|\Psi|^{2}\right] d y+\int \Pi w \cdot \nabla \Psi d y+\int \rho(y) Q \cdot \nabla \Psi d y \\
+\frac{1}{2} P \cdot B_{v} P-\frac{1}{2}\left\langle Q \cdot \nabla \psi_{v}(y), Q \cdot \nabla \rho(y)\right\rangle, \quad X=(\Psi, \Pi, Q, P) \in \mathscr{E} .
\end{aligned}
$$

ii) Закон сохранения энергии выполняется для решений $X(t) \in C(\mathbb{R}, \mathscr{E})$ : $\mathscr{H}_{v, w}(X(t))=$ const,$t \in \mathbb{R}$.

iii) Выполняются соотношения антисимметрии $\Omega\left(A_{v, w} X_{1}, X_{2}\right)=-\Omega\left(X_{1}\right.$, $\left.A_{v, w} X_{2}\right), X_{1}, X_{2} \in \mathscr{E}$.

Гамильтониан (5.15) можно получить, разлагая $\mathscr{H}\left(S_{b, v}+X\right)$ в степенной ряд по $X$ до членов второго порядка включительно. В итоге $\mathscr{H}_{v, w}(X)$ является квадратичной частью ряда Тейлора, дополненной вторым интегралом в правой части (5.15), который появляется из правой части (2.3).

Лемма 5.3 [25]. Оператор $A_{v, w}$ действует на касательные векторы $\tau_{j}(v)$ $\kappa$ солитонному многообразию следующим образом:

$$
\begin{aligned}
A_{v, w}\left[\tau_{j}(v)\right] & =(w-v) \cdot \nabla \tau_{j}(v), \\
A_{v, w}\left[\tau_{j+3}(v)\right] & =(w-v) \cdot \nabla \tau_{j+3}(v)+\tau_{j}(v), \quad j=1,2,3 .
\end{aligned}
$$

Мы применяем лемму 5.2 главным образом к оператору $A_{v, v}$, в котором $w=$ $v$. В этом случае линеаризованное уравнение имеет дополнительные важные свойства.

Лемма 5.4 [25]. Предположим, что $w=v \in V$. Тогда справедливы следующие утверждения.

i) Касательные векторы $\tau_{j}(v), j=1,2,3$, являются собственными векторами, а $\tau_{j+3}(v)$ - присоединенными векторами оператора $A_{v, v}$, отвечающими нулевому собственному значению, т.е.

$$
A_{v, v}\left[\tau_{j}(v)\right]=0, \quad A_{v, v}\left[\tau_{j+3}(v)\right]=\tau_{j}(v), \quad j=1,2,3 .
$$


ii) Гамильтониан (5.15) является неотрицательно определенным, а именно, $\mathscr{H}_{v, v}(X)=\frac{1}{2} \int\left[|\Pi+v \cdot \nabla \Psi|^{2}+\left|\Lambda^{1 / 2} \Psi-\Lambda^{-1 / 2} Q \cdot \nabla \rho\right|^{2}\right] d x+\frac{1}{2} P \cdot B_{v} P \geqslant 0$.

Здесъ $\Lambda$ - симметричный и неотрицательно определенный в $L^{2}\left(\mathbb{R}^{3}\right)$ при $|v|<1$ оператор (3.3), а $\Lambda^{1 / 2}$ - неотрицательный квадратный коренъ, определяемыи в представлении Фуръе.

Ниже мы применяем лемму 5.4, ii) вместе с сохранением энергии для доказательства аналитичности резольвенты $\left(A_{v, v}-\lambda\right)^{-1}$ в области $\operatorname{Re} \lambda>0$.

Для солитонного решения системы (1.1) имеем $\dot{b}=v, \dot{v}=0$, поэтому $T(t) \equiv 0$. В этом случае уравнение (5.13) является линеаризаиией системы (1.1). В общем случае, как мы покажем ниже, $T(t)$ является членом второго порядка малости по $Z(t)$, если выбрать $S(\sigma(t))$ симплектически ортогональной проекцией $Y(t)$. Тогда уравнение (5.13) снова окажется линеаризацией системы (1.1).

Для волнового уравнения все утверждения данного раздела остаются верными с заменой пространства $\mathscr{E}$ на $\dot{\mathscr{E}}$ и подстановкой $m=0$.

Для уравнения Максвелла лемма 5.3 остается верной, лемма 5.2 модифицируется следующим образом.

Лемма 5.5. і) Для любого $v$, где $|v|<1$, любого $w \in \mathbb{R}^{3}$ уравнение (5.13) является гамильтоновой системой

$$
\dot{X}(t)=J D \mathscr{H}_{v, w}(X(t)), \quad t \in \mathbb{R},
$$

где D $\mathscr{H}_{v, w}$ - производная Фреше гамильтониана

$$
\begin{aligned}
\mathscr{H}_{v, w}(X)=\frac{1}{2} \int\left[|e|^{2}+|\nabla a|^{2}\right] d y+\int a(w \cdot \nabla) e d y+\frac{1}{2}\left(B_{v}\langle\rho, a\rangle\right) \cdot\langle\rho, a\rangle \\
+\frac{1}{2} \pi \cdot B_{v} \pi+\langle r \cdot \nabla \rho v, a\rangle-\left\langle\rho B_{v} \pi, a\right\rangle \\
+\frac{1}{2}\left\langle r \cdot \nabla \rho, v \cdot\left(\nabla A_{v}\right)\right\rangle, \quad X=(e, a, r, \pi) \in \mathscr{E} .
\end{aligned}
$$

ii) Для решений $X(t) \in C(\mathbb{R}, \mathscr{E})$ выполняется закон сохранения энергии: $\mathscr{H}_{v, w}(X(t))=$ const, $t \in \mathbb{R}$.

iii) Выполняются соотношения антисимметрии $\Omega\left(A_{v, w} X_{1}, X_{2}\right)=-\Omega\left(X_{1}\right.$, $\left.A_{v, w} X_{2}\right), X_{1}, X_{2} \in \mathscr{E}$.

Лемма 5.4, с соответствующим изменением выражения для гамильтониана, остается верной.

\section{6. Симплектическое разложение динамики}

Разложим динамику на две компоненты: вдоль многообразия $\mathscr{S}$ и в трансверсальном направлении. Уравнение (5.3) получено без какого-либо предположения на $\sigma(t)$ из (5.1). Теперь мы собираемся выбрать $S(\sigma(t)):=\Pi Y(t)$, но для этого надо знать, что

$$
Y(t) \in \mathscr{O}_{\alpha}(\mathscr{S}), \quad t \in \mathbb{R},
$$


с некоторым $\mathscr{O}_{\alpha}(\mathscr{S})$, определенным в лемме 4.4. Это верно для $t=0$ согласно нашему основному предположению (3.8) с достаточно малым $d_{0}>0$. Тогда $S(\sigma(0))=\Pi Y(0)$, и $Z(0)=Y(0)-S(\sigma(0))$ определено. Ниже мы докажем, что (6.1) выполняется с $\alpha=-\beta$, если $d_{0}$ достаточно мало. Во-первых, из априорной оценки $(2.10)$ и леммы 4.4, iii) вытекает, что $\Pi Y(t)=S(\sigma(t))$, где $\sigma(t)=(b(t), v(t))$ и $|v(t)| \leqslant \widetilde{v}<1, t \in \mathbb{R}$, если $Y(t) \in \mathscr{O}_{-\beta}(\mathscr{S})$. Обозначим через $r_{-\beta}(\widetilde{v})$ положительное число из леммы 4.4, iv), соответствующее $\alpha=-\beta$. Тогда $S(\sigma)+Z \in \mathscr{O}_{-\beta}(\mathscr{S})$, если $\sigma=(b, v)$ с $|v|<\widetilde{v}$ и $\|Z\|_{-\beta}<r_{-\beta}(\widetilde{v})$. Заметим, что из (2.10) следует, что $\|Z(0)\|_{-\beta}<r_{-\beta}(\widetilde{v})$, если $d_{0}$ достаточно мало. Поэтому $S(\sigma(t))=\Pi Y(t)$ и $Z(t)=Y(t)-S(\sigma(t))$ определено для малых $t \geqslant 0$ таких, что $\|Z(t)\|_{-\beta}<r_{-\beta}(\widetilde{v})$. Это рассуждение формализуется следующим стандартным определением.

ОПРЕДЕЛЕНИЕ 6.1. $t_{*}$ - "момент выхода":

$$
t_{*}=\sup \left\{t>0:\|Z(s)\|_{-\beta}<r_{-\beta}(\widetilde{v}), 0 \leqslant s \leqslant t\right\},
$$

где $\beta \in \mathbb{R}$ для уравнения Клейна-Гордона и $\beta>-9 / 2$ для уравнений волнового и Максвелла.

Одна из наших главных целей - доказать, что $t_{*}=\infty$, если $d_{0}$ достаточно мало. Это будет выполнено, если мы покажем, что $\|Z(t)\|_{-\beta}<r_{-\beta}(\widetilde{v}) / 2,0 \leqslant$ $t<t_{*}$. Заметим, что $|Q(t)| \leqslant \bar{Q}:=r_{-\beta}(\widetilde{v}), 0 \leqslant t<t_{*}$. Поэтому $N(t)$ в $(5.3)$ удовлетворяет, согласно (5.6), следующей оценке:

$$
\|N(t)\|_{\beta} \leqslant C_{\beta}(\widetilde{v})\|Z(t)\|_{-\beta}^{2}, \quad 0 \leqslant t<t_{*} .
$$

Для волнового уравнения и уравнения Максвелла все рассуждения и определения этого раздела сохраняются при $-9 / 2<\beta \leqslant 0$ ввиду леммы 4.4. Оценка (6.3) выполняется при любом $\beta \in \mathbb{R}$ во всех случаях: для уравнения Клейна-Гордона, волнового и Максвелла.

\section{1. Динамика вдоль солитонного многообразия: модуляционные} уравнения. С этого момента мы фиксируем разложение $Y(t)=S(\sigma(t))+Z(t)$ для $0<t<t_{*}$, положив $S(\sigma(t))=\Pi Y(t)$. Такой выбор разложения позволяет значительно упростить асимптотический анализ динамических уравнений (5.3) для трансверсальной компоненты $Z(t)$. В качестве первого шага мы выведем уравнения конечномерной динамики вдоль солитонного многообразия, т. е. модуляционные уравнения для параметров $\sigma(t)$. С этой целью запишем условия симплектической ортогональности в виде

$$
\Omega\left(Z(t), \tau_{j}(t)\right)=0, \quad j=1, \ldots, 6, \quad 0 \leqslant t<t_{*},
$$

где векторы $\tau_{j}(t)=\tau_{j}(\sigma(t))$ суть базис касательного пространства $\mathscr{T}_{S(\sigma(t))} \mathscr{S}$. Заметим, что $\sigma(t)=(b(t), v(t))$, где $|v(t)| \leqslant \widetilde{v}<1,0 \leqslant t<t_{*}$, по лемме 4.4, iii). Удобно перейти к новым параметрам $(c, v)$ вместо $\sigma=(b, v)$, где

$$
c(t):=b(t)-\int_{0}^{t} v(\tau) d \tau, \quad \dot{c}(t)=\dot{b}(t)-v(t)=w(t)-v(t), \quad 0 \leqslant t<t_{*} .
$$


Нам потребуется не точный вид уравнений для $(c, v)$, а только следующее утверждение.

Лемма 6.2 [25]. Пусть $Y(t)$ - решение задачи Коши (2.1) и сделано разложение (5.1) при условии (6.4). Тогда $(c(t), v(t))$ удовлетворяют уравнению

$$
\left(\begin{array}{c}
\dot{c}(t) \\
\dot{v}(t)
\end{array}\right)=\mathscr{N}(\sigma(t), Z(t)), \quad 0 \leqslant t<t_{*},
$$

где $\mathscr{N}(\sigma, Z)=\mathscr{O}\left(\|Z\|_{-\beta}^{2}\right) \quad$ при любом $\beta \in \mathbb{R} \quad$ равномерно по $\sigma \in \Sigma(\widetilde{v})$.

ДокАзАтЕльство. Дифференцируя условия ортогональности (6.4) по $t$, получим

$$
0=\Omega\left(\dot{Z}, \tau_{j}\right)+\Omega\left(Z, \dot{\tau}_{j}\right)=\Omega\left(A Z+T+N, \tau_{j}\right)+\Omega\left(Z, \dot{\tau}_{j}\right), \quad 0 \leqslant t<t_{*} .
$$

Начнем с вычисления главного (т. е. не обращающегося в нуль при $Z=0$ ) члена $\Omega\left(T, \tau_{j}\right)$. Для $j=1,2,3$ ввиду $(5.7),(4.5)$ имеем:

$$
\Omega\left(T, \tau_{j}\right)=-\sum_{l}\left(\dot{c}_{l} \Omega\left(\tau_{l}, \tau_{j}\right)+\dot{v}_{l} \Omega\left(\tau_{l+3}, \tau_{j}\right)\right)=\sum_{l} \Omega\left(\tau_{j}, \tau_{l+3}\right) \dot{v}_{l}=\sum_{l} \Omega_{j l}^{+} \dot{v}_{l},
$$

где матрица $\Omega^{+}$определена в разделе 4. Аналогично,

$\Omega\left(T, \tau_{j+3}\right)=-\sum_{l}\left(\dot{c}_{l} \Omega\left(\tau_{l}, \tau_{j+3}\right)+\dot{v}_{l} \Omega\left(\tau_{l+3}, \tau_{j+3}\right)\right)=\sum_{l} \Omega\left(\tau_{j+3}, \tau_{l}\right) \dot{c}_{l}=-\sum_{l} \Omega_{j l}^{+} \dot{c}_{l}$.

В итоге в силу (4.5) получаем:

$$
\Omega(T, \tau)=\left(\begin{array}{cc}
0 & \Omega^{+}(v) \\
-\Omega^{+}(v) & 0
\end{array}\right)\left(\begin{array}{c}
\dot{c} \\
\dot{v}
\end{array}\right)=\Omega(v)\left(\begin{array}{c}
\dot{c} \\
\dot{v}
\end{array}\right) .
$$

Теперь найдем $\Omega\left(A Z, \tau_{j}\right)$. Ввиду антисимметричности, $\Omega\left(A Z, \tau_{j}\right)=-\Omega\left(Z, A \tau_{j}\right)$. Тогда для $j=1,2,3$ ввиду (5.16) получаем $\Omega\left(A Z, \tau_{j}\right)=-\Omega\left(Z, \dot{c} \cdot \nabla \tau_{j}\right)$ и, аналогично, $\Omega\left(A Z, \tau_{j+3}\right)=-\Omega\left(Z, \dot{c} \cdot \nabla \tau_{j+3}+\tau_{j}\right)=-\Omega\left(Z, \dot{c} \cdot \nabla \tau_{j+3}\right)-\Omega\left(Z, \tau_{j}\right)=$ $-\Omega\left(Z, \dot{c} \cdot \nabla \tau_{j+3}\right)$, поскольку $\Omega\left(Z, \tau_{j}\right)=0$.

Наконец, вычислим последний член $\Omega\left(Z, \dot{\tau}_{j}\right)$. Для $j=1, \ldots, 6$ имеем $\dot{\tau}_{j}=$ $\dot{b} \cdot \nabla_{b} \tau_{j}+\dot{v} \cdot \nabla_{v} \tau_{j}=\dot{v} \cdot \nabla_{v} \tau_{j}$, поскольку векторы $\tau_{j}$ не зависят от $b$ согласно (4.3). Поэтому $\Omega\left(Z, \dot{\tau}_{j}\right)=\Omega\left(Z, \dot{v} \cdot \nabla_{v} \tau_{j}\right)$. В результате уравнение $(6.7)$ записывается в виде

$$
0=\Omega(v)\left(\begin{array}{c}
\dot{c} \\
\dot{v}
\end{array}\right)+\mathscr{M}_{0}(\sigma, Z)\left(\begin{array}{c}
\dot{c} \\
\dot{v}
\end{array}\right)+\mathscr{N}_{0}(\sigma, Z),
$$

где $\mathscr{M}_{0}(\sigma, Z)=\mathscr{O}\left(\|Z\|_{-\beta}\right)$ и $\mathscr{N}_{0}(\sigma, Z)=\mathscr{O}\left(\|Z\|_{-\beta}^{2}\right)$ равномерно по $\sigma \in \Sigma(\widetilde{v})$ и $\|Z\|_{-\beta}<r_{-\beta}(\widetilde{v})$. Тогда, поскольку $\Omega(v)$ обратима в силу леммы 4.2 и $\|Z\|_{-\beta}$ мала, мы можем разрешить уравнение (6.8) относительно производных и получить уравнения (6.6) с $\mathscr{N}=\mathscr{O}\left(\|Z\|_{-\beta}^{2}\right)$ равномерно по $\sigma \in \Sigma(\widetilde{v})$. Лемма доказана.

В случае уравнений волнового и Максвелла разложение и соотношения симплектической ортогональности записываются аналогично. Лемма 6.2 выполняется с $\beta>-9 / 2$ в оценке (6.6). 
6.2. Убывание для трансверсальной динамики. В разделе 11 мы покажем, что основная теорема 3.3 выводится из следующей оценки временно́го убывания трансверсальной компоненты $Z(t)$.

ПрЕДЛОжЕНИЕ 6.3. Предположим, что выполнены все условия теоремъз 3.3. Тогда $t_{*}=\infty u$

$$
\|Z(t)\|_{-\beta} \leqslant \frac{C\left(\rho, \bar{v}, d_{0}\right)}{(1+|t|)^{3 / 2}}, \quad t \geqslant 0 .
$$

Оценка (6.9) выводится из нашего уравнения (5.3) для трансверсальной компоненты $Z(t)$. Это уравнение можно уточнить, используя лемму 6.2. Действительно, из леммы следует, что $\|T(t)\|_{\beta} \leqslant C(\widetilde{v})\|Z(t)\|_{-\beta}^{2}, 0 \leqslant t<t_{*}$, ввиду (5.5), поскольку $w-v=\dot{c}$. Таким образом, уравнение (5.3) преобразуется в уравнение

$$
\dot{Z}(t)=A(t) Z(t)+\tilde{N}(t), \quad 0 \leqslant t<t_{*},
$$

где $A(t)=A_{v(t), w(t)}$ и $\widetilde{N}(t):=T(t)+N(t)$ удовлетворяет оценке

$$
\|\tilde{N}(t)\|_{\beta} \leqslant C\|Z(t)\|_{-\beta}^{2}, \quad 0 \leqslant t<t_{*} .
$$

Ниже мы изучаем главным образом это основное уравнение (6.10), чтобы вывести убывание (6.9). Убывание будет получено на основе оценки (6.11) и условий ортогональности (6.4).

Прокомментируем две основные трудности в доказательстве (6.9), общие для задач того типа, которые изучаются в [33]. Во-первых, линейная часть уравнения является неавтономной, поэтому мы не можем применить непосредственно известные методы теории рассеяния. Аналогично подходу [33], мы сводим задачу к анализу замороженного линейного уравнения

$$
\dot{X}(t)=A_{1} X(t), \quad t \in \mathbb{R},
$$

где $A_{1}$ - оператор $A_{v_{1}, v_{1}}$, определенный формулой (5.4) с $v_{1}=v\left(t_{1}\right)$ для некоторого фиксированного $t_{1} \in\left[0, t_{*}\right)$. Затем мы оцениваем невязку методом мажсорант.

Во-вторых, даже для замороженного уравнения (6.12) убывание типа (6.9) для всех решений не выполняется, если не наложить условий ортогональности типа (6.4). А именно, согласно (5.17), уравнение (6.12) имеет секулярные решения

$$
X(t)=\sum_{1}^{3} C_{j} \tau_{j}\left(v_{1}\right)+\sum_{1}^{3} D_{j}\left[\tau_{j}\left(v_{1}\right) t+\tau_{j+3}\left(v_{1}\right)\right] .
$$

Значит, чтобы избежать секулярных решений, мы должны учесть условия ортогональности (6.4). С этой целью мы применяем соответствующую симплектически ортогональную проекцию, которая устраняет "убегающие решения" (6.13).

Решение (6.13) лежит в касательном пространстве $\mathscr{T}_{S\left(\sigma_{1}\right)} \mathscr{S}$ с $\sigma_{1}=\left(b_{1}, v_{1}\right)$ (для произвольного $b_{1} \in \mathbb{R}$ ), что означает неустойчивый характер нелинейной динамики вдоль солитонного многообразия. 
ОПРЕДЕЛЕНИЕ 6.4. і) Для $v \in V$ обозначим через $\boldsymbol{\Pi}_{v}$ симплектически ортогональную проекцию $\mathscr{E}$ на касательное пространство $\mathscr{T}_{S(\sigma)} \mathscr{S}$, положим $\mathbf{P}_{v}=$ $\mathbf{I}-\boldsymbol{\Pi}_{v}$.

ii) Обозначим через $\mathscr{Z}_{v}=\mathbf{P}_{v} \mathscr{E}$ пространство, симплектически ортогональное к $\mathscr{T}_{S(\sigma)} \mathscr{S}$ с $\sigma=(b, v)$ (для произвольного $b \in \mathbb{R}$ ).

Заметим, что, ввиду линейности,

$$
\boldsymbol{\Pi}_{v} Z=\sum \boldsymbol{\Pi}_{j l}(v) \tau_{j}(v) \Omega\left(\tau_{l}(v), Z\right), \quad Z \in \mathscr{E},
$$

с некоторыми гладкими коэффициентами $\boldsymbol{\Pi}_{j l}(v)$. Поэтому проектор $\boldsymbol{\Pi}_{v}$, записанный в переменной $y=x-b$, не зависит от $b,-$ это объясняет выбор нижнего индекса в $\boldsymbol{\Pi}_{v}$ и $\mathbf{P}_{v}$.

Мы имеем симплектически ортогональное разложение

$$
\mathscr{E}=\mathscr{T}_{S(\sigma)} \mathscr{S}+\mathscr{Z}_{v}, \quad \sigma=(b, v) .
$$

Теперь симплектическую ортогональность (6.4) можно записать в следующих эквивалентных формах:

$$
\boldsymbol{\Pi}_{v(t)} Z(t)=0, \quad \mathbf{P}_{v(t)} Z(t)=Z(t), \quad 0 \leqslant t<t_{*} .
$$

Касательное пространство $\mathscr{T}_{S(\sigma)} \mathscr{S}$ инвариантно относительно оператора $A_{v, v}$ по лемме 5.4, i), поэтому пространство $\mathscr{Z}_{v}$ тоже инвариантно, ввиду соотношений антисимметрии: $A_{v, v} Z \in \mathscr{Z}_{v}$ на плотном подмножестве $Z \in \mathscr{Z}_{v}$.

Ниже мы докажем следующее предложение, которое является одной из главных составляющих для вывода (6.9). Рассмотрим задачу Коши для уравнения (6.12) с $A=A_{v, v}$ при фиксированном $v \in V$. Напомним, что параметр $\beta>3 / 2$ также фиксирован.

ПРЕДЛОЖЕНИЕ 6.5. Пусть выполнены условия (2.8) u (1.9), $|v| \leqslant \widetilde{v}<1 u$ $X_{0} \in \mathscr{E}$. Тогда:

i) уравнение (6.12) с $A_{1}=A=A_{v, v}$ имеет единственное решение $e^{A t} X_{0}:=$ $X(t) \in C(\mathbb{R}, \mathscr{E})$ с начальным условием $X(0)=X_{0}$;

ii) для $X_{0} \in \mathscr{Z}_{v} \cap \mathscr{E}_{\beta}$ решение $X(t)$ имеет следующее убывание:

$$
\left\|e^{A t} X_{0}\right\|_{-\beta} \leqslant \frac{C(\beta, \widetilde{v})}{(1+|t|)^{3 / 2}}\left\|X_{0}\right\|_{\beta}, \quad t \in \mathbb{R} .
$$

Убывание обеспечивается двумя фундаментальными фактами, которые будут установлены ниже: нулевое корневое подпространство генератора $A$ совпадает с касательным пространством $\mathscr{T}_{S(\sigma)} \mathscr{S}$, где $\sigma=(b, v)(b \in \mathbb{R}$ произвольно), и спектр оператора $A$ в пространстве $\mathscr{Z}_{v}$ является чисто непрерывным.

Для случаев волнового уравнения и уравнения Максвелла оценка убывания трансверсальной компоненты имеет следующий вид.

ПРЕДЛОЖЕНИЕ 6.6. Предположим, что выполнены все условия теоремы 3.4 (теоремы 3.5). Тогда $t_{*}=\infty u$

$$
\|Z(t)\|_{-\beta} \leqslant \frac{C\left(\rho, \bar{v}, d_{\delta}\right)}{(1+|t|)^{1+\delta}}, \quad t \geqslant 0, \quad \beta=4+\delta .
$$


Предложение 6.5 принимает следующий вид.

ПРЕДЛОЖЕНИЕ 6.7. Пусть выполненъ условия (2.8), (1.9), (1.10), $\left|v_{1}\right| \leqslant$ $\widetilde{v}<1$ u $X_{0} \in \dot{\mathscr{E}}$. Тогда:

i) уравнение $(6.12)$ с $A_{1}=A_{v_{1}, v_{1}}$ имеет единственное решение $e^{A_{1} t} X_{0}:=$ $X(t) \in C(\mathbb{R}, \mathscr{E})$ с начальным условием $X(0)=X_{0}$;

ii) если $X_{0}=\mathbf{P}_{v_{1}} Z_{0}, Z_{0} \in \dot{\mathscr{E}}_{\beta}$, то решение $X(t)$ убывает следующим образом:

$$
\left\|e^{A_{1} t} X_{0}\right\|_{-(2+\delta)} \leqslant \frac{C_{\delta}(\widetilde{v})}{(1+|t|)^{1+\delta}}\left\|X_{0}\right\|_{\beta}, \quad t \in \mathbb{R}, \quad \beta=4+\delta .
$$

\section{7. Метод мажорант}

7.1. “Замораживание” трансверсальной динамики. Зафиксируем произвольное $t_{1} \in\left[0, t_{*}\right)$ и перепишем уравнение $(6.10)$ в "замороженном" виде

$$
\dot{Z}(t)=A_{1} Z(t)+\left(A(t)-A_{1}\right) Z(t)+\tilde{N}(t), \quad 0 \leqslant t<t_{*},
$$

где $A_{1}=A_{v\left(t_{1}\right), v\left(t_{1}\right)}$ и

$$
A(t)-A_{1}=\left(\begin{array}{cccc}
{\left[w(t)-v\left(t_{1}\right)\right] \cdot \nabla} & 0 & 0 & 0 \\
0 & {\left[w(t)-v\left(t_{1}\right)\right] \cdot \nabla} & 0 & 0 \\
0 & 0 & 0 & B_{v(t)}-B_{v\left(t_{1}\right)} \\
0 & 0 & \left\langle\nabla\left(\psi_{v(t)}-\psi_{v\left(t_{1}\right)}\right), \nabla \rho\right\rangle & 0
\end{array}\right) .
$$

Для уничтожения "плохих" членов $\left[w(t)-v\left(t_{1}\right)\right] \cdot \nabla$ выполним замену переменных $(y, t) \mapsto\left(y_{1}, t\right)=\left(y+d_{1}(t), t\right)$, где

$$
d_{1}(t):=\int_{t_{1}}^{t}\left(w(s)-v\left(t_{1}\right)\right) d s, \quad 0 \leqslant t \leqslant t_{1} .
$$

Теперь определим $Z_{1}(t)=\left(\Psi\left(y_{1}-d_{1}(t), t\right), \Pi\left(y_{1}-d_{1}(t), t\right), Q(t), P(t)\right)$. Тогда мы получим окончательную форму “замороженного” уравнения для трансверсальной динамики:

$$
\dot{Z}_{1}(t)=A_{1} Z_{1}(t)+B_{1}(t) Z_{1}(t)+N_{1}(t), \quad 0 \leqslant t \leqslant t_{1},
$$

где $N_{1}(t)=\tilde{N}(t)$ выражено в терминах $y=y_{1}-d_{1}(t)$, а

$$
B_{1}(t)=\left(\begin{array}{cccc}
0 & 0 & 0 & 0 \\
0 & 0 & 0 & 0 \\
0 & 0 & 0 & B_{v(t)}-B_{v\left(t_{1}\right)} \\
0 & 0 & \left\langle\nabla\left(\psi_{v(t)}-\psi_{v\left(t_{1}\right)}\right), \nabla \rho\right\rangle & 0
\end{array}\right) .
$$

Выведем оценки для "остаточных" членов $B_{1}(t) Z_{1}(t)$ и $N_{1}(t)$ в $(7.3)$. Во-первых, заметим, что по лемме 6.2 мы имеем:

$$
\left|B_{v(t)}-B_{v\left(t_{1}\right)}\right| \leqslant\left|\int_{t_{1}}^{t} \dot{v}(s) \cdot \nabla_{v} B_{v(s)} d s\right| \leqslant C \int_{t}^{t_{1}}\|Z(s)\|_{-\beta}^{2} d s .
$$


Аналогично,

$$
\left|\left\langle\nabla\left(\psi_{v(t)}-\psi_{v\left(t_{1}\right)}\right), \nabla \rho\right\rangle\right| \leqslant C \int_{t}^{t_{1}}\|Z(s)\|_{-\beta}^{2} d s .
$$

При помощи хорошо известного неравенства $(1+|y+x|)^{\alpha} \leqslant(1+|y|)^{\alpha}(1+|x|)^{|\alpha|}$, $x, y \in \mathbb{R}^{3}, \alpha \in \mathbb{R}$, выводится следующее утверждение.

Лемма 7.1 [25]. Для $(\Psi, \Pi, Q, P) \in \mathscr{E}_{\alpha}$ с любим $\alpha \in \mathbb{R}$ выполняется оценка

$$
\left\|\left(\Psi\left(y_{1}-d_{1}\right), \Pi\left(y_{1}-d_{1}\right), Q, P\right)\right\|_{\alpha} \leqslant\|(\Psi, \Pi, Q, P)\|_{\alpha}\left(1+\left|d_{1}\right|\right)^{|\alpha|}, \quad d_{1} \in \mathbb{R}^{3} .
$$

Как следствие получаются оценки

$$
\begin{array}{cc}
\left\|N_{1}(t)\right\|_{\beta} \leqslant\left(1+\left|d_{1}(t)\right|\right)^{3 \beta}\|Z(t)\|_{-\beta}^{2}, & 0 \leqslant t \leqslant t_{1}, \\
\left\|B_{1}(t) Z_{1}(t)\right\|_{\beta} \leqslant C\left\|Z_{1}(t)\right\|_{-\beta} \int_{t}^{t_{1}}\left(1+\left|d_{1}(t)\right|\right)^{2 \beta}\left\|Z_{1}(\tau)\right\|_{-\beta}^{2} d \tau, & 0 \leqslant t \leqslant t_{1} .
\end{array}
$$

В случае уравнения Клейна-Гордона оценки (7.7) и (7.8) выполняются с $\beta>3 / 2$. Аналогичные оценки выполнены также для случаев волнового уравнения и уравнения Максвелла с $\beta=4+\delta$.

7.2. Интегральное неравенство. Уравнение (7.3) можно записать в интегральной форме:

$$
Z_{1}(t)=e^{A_{1} t} Z_{1}(0)+\int_{0}^{t} e^{A_{1}(t-s)}\left[B_{1} Z_{1}(s)+N_{1}(s)\right] d s, \quad 0 \leqslant t \leqslant t_{1} .
$$

Применяя симплектически ортогональную проекцию $\mathbf{P}_{1}:=\mathbf{P}_{v\left(t_{1}\right)}$ к обеим частям, получим

$$
\mathbf{P}_{1} Z_{1}(t)=e^{A_{1} t} \mathbf{P}_{1} Z_{1}(0)+\int_{0}^{t} e^{A_{1}(t-s)} \mathbf{P}_{1}\left[B_{1} Z_{1}(s)+N_{1}(s)\right] d s .
$$

Здесь мы воспользовались тем, что $\mathbf{P}_{1}$ коммутирует с группой $e^{A_{1} t}$, поскольку пространство $\mathscr{Z}_{1}:=\mathbf{P}_{1} \mathscr{E}$ инвариантно относительно $e^{A_{1} t}$. Применяя (6.17), мы получим, что

$$
\begin{aligned}
\left\|\mathbf{P}_{1} Z_{1}(t)\right\|_{-\beta} \leqslant & \frac{C}{(1+t)^{3 / 2}}\left\|\mathbf{P}_{1} Z_{1}(0)\right\|_{\beta} \\
& +C \int_{0}^{t} \frac{1}{(1+|t-s|)^{3 / 2}}\left\|\mathbf{P}_{1}\left[B_{1} Z_{1}(s)+N_{1}(s)\right]\right\|_{\beta} d s .
\end{aligned}
$$

Оператор $\mathbf{P}_{1}=\mathbf{I}-\boldsymbol{\Pi}_{1}$ непрерывен на $\mathscr{E}_{\beta}$ согласно (6.14). Следовательно, из (7.10) и (7.7), (7.8) мы получаем, что при $0 \leqslant t \leqslant t_{1}$

$$
\begin{aligned}
\left\|\mathbf{P}_{1} Z_{1}(t)\right\|_{-\beta} & \leqslant \frac{C\left(\bar{d}_{1}(0)\right)}{(1+t)^{3 / 2}}\|Z(0)\|_{\beta} \\
& +\int_{0}^{t} \frac{C\left(\bar{d}_{1}(t)\right)}{(1+|t-s|)^{3 / 2}}\left[\|Z(s)\|_{-\beta} \int_{s}^{t_{1}}\|Z(\tau)\|_{-\beta}^{2} d \tau+\|Z(s)\|_{-\beta}^{2}\right] d s
\end{aligned}
$$


где $\bar{d}_{1}(t):=\sup _{0 \leqslant s \leqslant t}\left|d_{1}(s)\right|$. Введем мажоранту

$$
m(t):=\sup _{s \in[0, t]}(1+s)^{3 / 2}\|Z(s)\|_{-\beta}, \quad t \in\left[0, t_{*}\right) .
$$

Чтобы оценить $d_{1}(t)$ через $m\left(t_{1}\right)$, заметим, что

$$
w(s)-v\left(t_{1}\right)=w(s)-v(s)+v(s)-v\left(t_{1}\right)=\dot{c}(s)+\int_{s}^{t_{1}} \dot{v}(\tau) d \tau
$$

ввиду (6.5). Тогда из (7.2), леммы 6.2 и определения (7.12) следует, что при $0 \leqslant t \leqslant t_{1}$

$$
\begin{aligned}
\left|d_{1}(t)\right| & \leqslant \int_{t}^{t_{1}}\left(|\dot{c}(s)|+\int_{s}^{t_{1}}|\dot{v}(\tau)| d \tau\right) d s \\
& \leqslant C m^{2}\left(t_{1}\right) \int_{t}^{t_{1}}\left(\frac{1}{(1+s)^{3}}+\int_{s}^{t_{1}} \frac{d \tau}{(1+\tau)^{3}}\right) d s \leqslant C m^{2}\left(t_{1}\right) .
\end{aligned}
$$

Мы сможем в (7.11) заменить константу $C\left(\bar{d}_{1}\right)$ на $C$, если $m\left(t_{1}\right)$ будет ограничена при $t_{1} \geqslant 0$. Чтобы выполнить эту замену, введем еще один момент выхода. Обозначим через $\varepsilon$ некоторое фиксированное положительное число, которое ниже будет выбрано достаточно малым, см. лемму 7.3.

ОПРЕДЕЛЕНИЕ 7.2. $t_{*}^{\prime}-$ момент выхода

$$
t_{*}^{\prime}=\sup \left\{t \in\left[0, t_{*}\right): m(s) \leqslant \varepsilon, 0 \leqslant s \leqslant t\right\} .
$$

Теперь из $(7.11)$ при $0 \leqslant t \leqslant t_{1}<t_{*}^{\prime}$ вытекает неравенство

$$
\begin{aligned}
\left\|\mathbf{P}_{1} Z_{1}(t)\right\|_{-\beta} \leqslant & \frac{C\|Z(0)\|_{\beta}}{(1+t)^{3 / 2}}+\int_{0}^{t} \frac{C}{(1+|t-s|)^{3 / 2}} \\
& \times\left[\|Z(s)\|_{-\beta} \int_{s}^{t_{1}}\|Z(\tau)\|_{-\beta}^{2} d \tau+\|Z(s)\|_{-\beta}^{2}\right] d s .
\end{aligned}
$$

Для волнового уравнения и уравнения Максвелла при помощи аналогичных рассуждений получаем следующую оценку типа (7.16) с $\beta=4+\delta$ : при $0 \leqslant t \leqslant t_{1}$

$$
\begin{aligned}
\left\|\mathbf{P}_{1} Z_{1}(t)\right\|_{-(2+\delta)} \leqslant & \frac{C\|Z(0)\|_{\beta}}{(1+t)^{1+\delta}}+\int_{0}^{t} \frac{C}{(1+|t-s|)^{1+\delta}} \\
& \times\left[\|Z(s)\|_{-\beta} \int_{s}^{t_{1}}\|Z(\tau)\|_{-\beta}^{2} d \tau+\|Z(s)\|_{-\beta}^{2}\right] d s .
\end{aligned}
$$

7.3. Симплектическая ортогональность. Наконец, мы собираемся заменить $\mathbf{P}_{1} Z_{1}(t)$ на $Z(t)$ в левой части (7.16). Мы покажем, что это возможно ввиду следующей важной оценки.

ЛЕмма 7.3. При достаточно малом $\varepsilon>0$ для $t_{1}<t_{*}^{\prime}$ мы имеем:

$$
\|Z(t)\|_{-\beta} \leqslant C\left\|\mathbf{P}_{1} Z_{1}(t)\right\|_{-\beta}, \quad 0 \leqslant t \leqslant t_{1}, \quad C=C(\rho, \bar{v}) .
$$


ДокАЗАтЕльство. Доказательство основано на симплектической ортогональности $(6.16)$, т. е. $\boldsymbol{\Pi}_{v(t)} Z(t)=0, t \in\left[0, t_{1}\right]$, и на том факте, что все пространства $\mathscr{Z}(t):=\mathbf{P}_{v(t)} \mathscr{E}$ "почти параллельны" друг другу при всех $t$. А именно, сначала заметим, что $\|Z(t)\|_{-\beta} \leqslant C\left\|Z_{1}(t)\right\|_{-\beta}$ по лемме 7.1 , поскольку $\left|d_{1}(t)\right| \leqslant 1$ для $t \leqslant t_{1}<t_{*}^{\prime \prime}<t_{*}^{\prime}$. Поэтому достаточно доказать, что $\left\|Z_{1}(t)\right\|_{-\beta} \leqslant$ $2\left\|\mathbf{P}_{1} Z_{1}(t)\right\|_{-\beta}, 0 \leqslant t \leqslant t_{1}$. Эта оценка будет следовать из оценки

$$
\left\|\boldsymbol{\Pi}_{v\left(t_{1}\right)} Z_{1}(t)\right\|_{-\beta} \leqslant \frac{1}{2}\left\|Z_{1}(t)\right\|_{-\beta}, \quad 0 \leqslant t \leqslant t_{1},
$$

поскольку $\mathbf{P}_{1} Z_{1}(t)=Z_{1}(t)-\boldsymbol{\Pi}_{v\left(t_{1}\right)} Z_{1}(t)$. Чтобы доказать (7.19), запишем условия ортогональности в виде $\boldsymbol{\Pi}_{v(t), 1} Z_{1}(t)=0, t \in\left[0, t_{1}\right]$, где $\boldsymbol{\Pi}_{v(t), 1} Z_{1}(t)$ - это $\boldsymbol{\Pi}_{v(t)} Z(t)$, выраженное через переменную $y_{1}=y+d_{1}(t)$. Значит, мы получим (7.19), если разность $\boldsymbol{\Pi}_{v\left(t_{1}\right)}-\boldsymbol{\Pi}_{v(t), 1}$ мала равномерно по $t$, т. е.

$$
\left\|\boldsymbol{\Pi}_{v\left(t_{1}\right)}-\boldsymbol{\Pi}_{v(t), 1}\right\|<\frac{1}{2}, \quad 0 \leqslant t \leqslant t_{1} .
$$

Остается обосновать (7.20) для достаточно малого $\varepsilon>0$. Для этого нам потребуется формула (6.14), а также следующее соотношение, которое вытекает из (6.14):

$$
\boldsymbol{\Pi}_{v(t), 1} Z_{1}(t)=\sum \boldsymbol{\Pi}_{j l}(v(t)) \tau_{j, 1}(v(t)) \Omega\left(\tau_{l, 1}(v(t)), Z_{1}(t)\right),
$$

где $\tau_{j, 1}(v(t))$ - векторы $\tau_{j}(v(t))$, выраженные через переменные $y_{1}$. Подробнее (см. $(4.3))$,

$$
\begin{aligned}
\tau_{j, 1}(v) & :=\left(-\partial_{j} \psi_{v}\left(y_{1}-d_{1}(t)\right),-\partial_{j} \pi_{v}\left(y_{1}-d_{1}(t)\right), e_{j}, 0\right), \\
\tau_{j+3,1}(v) & :=\left(\partial_{v_{j}} \psi_{v}\left(y_{1}-d_{1}(t)\right), \partial_{v_{j}} \pi_{v}\left(y_{1}-d_{1}(t)\right), 0, \partial_{v_{j}} p_{v}\right), \quad j=1,2,3,
\end{aligned}
$$

где $v=v(t)$. Поскольку $\left|d_{1}(t)\right| \leqslant 1$, а $\nabla \tau_{j}$ - достаточно гладкие и быстро убывающие на бесконечности функции, из леммы 7.1 следует, что

$$
\left\|\tau_{j, 1}(v(t))-\tau_{j}(v(t))\right\|_{\beta} \leqslant C\left|d_{1}(t)\right|^{\beta}, \quad 0 \leqslant t \leqslant t_{1},
$$

для всех $j=1, \ldots, 6$. Далее,

$$
\tau_{j}(v(t))-\tau_{j}\left(v\left(t_{1}\right)\right)=\int_{t}^{t_{1}} \dot{v}(s) \cdot \nabla_{v} \tau_{j}(v(s)) d s
$$

и поэтому

$$
\left\|\tau_{j}(v(t))-\tau_{j}\left(v\left(t_{1}\right)\right)\right\|_{\beta} \leqslant C \int_{t}^{t_{1}}|\dot{v}(s)| d s, \quad 0 \leqslant t \leqslant t_{1} .
$$

Аналогично,

$\left|\boldsymbol{\Pi}_{j l}(v(t))-\boldsymbol{\Pi}_{j l}\left(v\left(t_{1}\right)\right)\right|=\left|\int_{t}^{t_{1}} \dot{v}(s) \cdot \nabla_{v} \boldsymbol{\Pi}_{j l}(v(s)) d s\right| \leqslant C \int_{t}^{t_{1}}|\dot{v}(s)| d s, \quad 0 \leqslant t \leqslant t_{1}$,

поскольку $\left|\nabla_{v} \boldsymbol{\Pi}_{j l}(v(s))\right|$ равномерно ограничено, так как $|v(s)| \leqslant \bar{v}<1$. Поэтому оценки (7.20) будут вытекать из (6.14), (7.21) и (7.23)-(7.25), если мы 
покажем, что интеграл в правой части формул (7.24) и (7.25) можно сделать сколь угодно малым, если взять $\varepsilon>0$ достаточно малым. Действительно,

$$
\int_{t}^{t_{1}}|\dot{v}(s)| d s \leqslant C m^{2}\left(t_{1}\right) \int_{t}^{t_{1}} \frac{d s}{(1+s)^{3}} \leqslant C \varepsilon^{2}, \quad 0 \leqslant t \leqslant t_{1} .
$$

Лемма доказана.

В случае волнового уравнения и уравнения Максвелла оценка (7.18) леммы 7.3 принимает вид

$$
\|Z(t)\|_{-(2+\delta)} \leqslant C\left\|\mathbf{P}_{1} Z_{1}(t)\right\|_{-(2+\delta)}, \quad 0 \leqslant t \leqslant t_{1},
$$

и доказывается аналогично.

7.4. Убывание трансверсальной компоненты. Завершим доказательство предложения 6.3.

Шаг і). Зафиксируем такие $\varepsilon>0$ и $t_{*}^{\prime}=t_{*}^{\prime}(\varepsilon)$, для которых имеет место лемма 7.3. Тогда выполняется оценка типа (7.16), где $\left\|\mathbf{P}_{1} Z_{1}(t)\right\|_{-\beta}$ в левой части заменено на $\|Z(t)\|_{-\beta}$ : при $0 \leqslant t \leqslant t_{1}$

$$
\begin{aligned}
\|Z(t)\|_{-\beta} & \leqslant \frac{C\|Z(0)\|_{\beta}}{(1+t)^{3 / 2}} \\
& +\int_{0}^{t} \frac{C}{(1+|t-s|)^{3 / 2}}\left[\|Z(s)\|_{-\beta} \int_{s}^{t_{1}}\|Z(\tau)\|_{-\beta}^{2} d \tau+\|Z(s)\|_{-\beta}^{2}\right] d s
\end{aligned}
$$

для $t_{1}<t_{*}^{\prime}$. Отсюда следует интегральное неравенство для мажоранты

$$
m(t):=\sup _{s \in[0, t]}(1+s)^{3 / 2}\|Z(s)\|_{-\beta} .
$$

А именно, умножая обе части $(7.28)$ на $(1+t)^{3 / 2}$ и беря супремум по $t \in\left[0, t_{1}\right]$, мы получим

$$
\begin{aligned}
m\left(t_{1}\right) \leqslant C & \|Z(0)\|_{\beta}+C \sup _{t \in\left[0, t_{1}\right]} \int_{0}^{t} \frac{(1+t)^{3 / 2}}{(1+|t-s|)^{3 / 2}} \\
\times & {\left[\frac{m(s)}{(1+s)^{3 / 2}} \int_{s}^{t_{1}} \frac{m^{2}(\tau) d \tau}{(1+\tau)^{3}}+\frac{m^{2}(s)}{(1+s)^{3}}\right] d s }
\end{aligned}
$$

для $t_{1}<t_{*}^{\prime}$. Учитывая, что $m(t)-$ монотонно возрастающая функция, приходим к оценке $m\left(t_{1}\right) \leqslant C\|Z(0)\|_{\beta}+C\left[m^{3}\left(t_{1}\right)+m^{2}\left(t_{1}\right)\right] I\left(t_{1}\right), t_{1}<t_{*}^{\prime}$, где

$$
\begin{aligned}
I\left(t_{1}\right)= & \sup _{t \in\left[0, t_{1}\right]} \int_{0}^{t} \frac{(1+t)^{3 / 2}}{(1+|t-s|)^{3 / 2}} \\
& \times\left[\frac{1}{(1+s)^{3 / 2}} \int_{s}^{t_{1}} \frac{d \tau}{(1+\tau)^{3}}+\frac{1}{(1+s)^{3}}\right] d s \leqslant \bar{I}<\infty, \quad t_{1} \geqslant 0 .
\end{aligned}
$$

Отсюда следует, что $m\left(t_{1}\right) \leqslant C\|Z(0)\|_{\beta}+C \bar{I}\left[m^{3}\left(t_{1}\right)+m^{2}\left(t_{1}\right)\right], t_{1}<t_{*}^{\prime}$. Из этого неравенства вытекает, что $m\left(t_{1}\right)$ ограничено для $t_{1}<t_{*}^{\prime}$ и, более того,

$$
m\left(t_{1}\right) \leqslant C_{1}\|Z(0)\|_{\beta}, \quad t_{1}<t_{*}^{\prime},
$$

поскольку $m(0)=\|Z(0)\|_{\beta}$ достаточно мало в силу (4.13). 
Шаг іi). Постоянная $C_{1}$ в оценке (7.29) не зависит от $t_{*}$ и $t_{*}^{\prime}$ по лемме 7.3. Выберем $d_{\delta}$ в (3.8) столь малым, что $\|Z(0)\|_{\beta}<\varepsilon /\left(2 C_{1}\right)$. Такой выбор возможен в силу (4.13). Тогда из оценки (7.29) следует, что $t_{*}^{\prime}=t_{*}$, поэтому $(7.29)$ выполняется при всех $t_{1}<t_{*}$. Тогда оценка (7.14) выполняется при всех $t<t_{*}$. Значит, и оценка (7.29) выполняется при всех $t_{1}<t_{*}$. Отсюда следует, что $t_{*}=\infty$, тогда и $t_{*}^{\prime}=\infty$, и (7.29) выполняется при всех $t_{1}>0$, если $d_{\delta}$ достаточно мало. Предложение 6.3 доказано.

Для уравнений волнового и Максвелла завершение доказательства предложения 6.6 аналогично.

\section{8. Солитонная асимптотика}

Мы докажем основную теорему 3.3 в предположении, что имеет место убывание (6.9). Сначала мы установим асимптотику (3.9) для векторных компонент, а затем асимптотику (3.3) для полей.

Имеем $\dot{q}=\dot{b}+\dot{Q}$, а из $(6.10),(6.11),(5.4)$ следует, что $\dot{Q}=B_{v(t)} P+\mathscr{O}\left(\|Z\|_{-\beta}^{2}\right)$. Таким образом, $\dot{q}=\dot{b}+\dot{Q}=v(t)+\dot{c}(t)+B_{v(t)} P(t)+\mathscr{O}\left(\|Z\|_{-\beta}^{2}\right)$. Из (6.6), (6.9) следует, что $|\dot{c}(t)|+|\dot{v}(t)| \leqslant C_{1}\left(\rho, \bar{v}, d_{0}\right) /(1+t)^{3}, t \geqslant 0$. Поэтому $c(t)=c_{+}+\mathscr{O}\left(t^{-2}\right)$ и $v(t)=v_{+}+\mathscr{O}\left(t^{-2}\right), t \rightarrow \infty$. Поскольку $|P| \leqslant\|Z\|_{-\beta}$, из оценки (6.9) следует, что

$$
\dot{q}(t)=v_{+}+\mathscr{O}\left(t^{-3 / 2}\right)
$$

Аналогично,

$$
b(t)=c(t)+\int_{0}^{t} v(s) d s=v_{+} t+a_{+}+\mathscr{O}\left(t^{-1}\right) .
$$

Поскольку $Q(t)=\mathscr{O}\left(t^{-3 / 2}\right)$ ввиду $(6.9)$, это влечет вторую часть асимптотики (3.9):

$$
q(t)=b(t)+Q(t)=v_{+} t+a_{+}+\mathscr{O}\left(t^{-1}\right)
$$

В случае волнового уравнения и уравнения Максвелла имеем $|\dot{c}(t)|+|\dot{v}(t)| \leqslant$ $C_{1}\left(\rho, \bar{v}, d_{\beta}\right) /(1+t)^{2+2 \delta}, t \geqslant 0$. Тогда $c(t)=c_{+}+\mathscr{O}\left(t^{-(1+2 \delta)}\right)$ и $v(t)=v_{+}+$ $\mathscr{O}\left(t^{-(1+2 \delta)}\right)$, асимптотика для $\dot{q}$ примет вид

$$
\dot{q}(t)=v_{+}+\mathscr{O}\left(t^{-(1+\delta)}\right)
$$

Далее,

$$
b(t)=c(t)+\int_{0}^{t} v(s) d s=v_{+} t+a_{+}+\mathscr{O}\left(t^{-2 \delta}\right),
$$

что влечет вторую часть асимптотики (3.9):

$$
q(t)=v_{+} t+a_{+}+\mathscr{O}\left(t^{-2 \delta}\right) .
$$

Теперь выведем асимптотики для полей. Мы применяем подход работы [18]. Для полевой части решения $F(t)=(\psi(x, t), \pi(x, t))$ в исходной переменной $x$ определим сопутствующее солитонное поле формулой $F_{\mathrm{v}(\mathrm{t})}(t)=\left(\psi_{\mathrm{v}(\mathrm{t})}(x-q(t))\right.$, $\left.\pi_{\mathrm{v}(\mathrm{t})}(x-q(t))\right)$, где теперь мы положим $\mathrm{v}(t)=\dot{q}(t)$. Тогда для разности $Z(t)=$ 
$F(t)-F_{\mathrm{v}(\mathrm{t})}(t)$ из первых двух уравнений системы (1.1) следует неоднородное уравнение Клейна-Гордона (см. [18; формула $(2.5)]) \dot{Z}(t)=A_{0} Z(t)-\dot{\mathrm{v}}$. $\nabla_{\mathrm{v}} F_{\mathrm{v}(t)}(t), A_{0}(\psi, \pi)=\left(\pi,\left(\Delta-m^{2}\right) \psi\right)$. Тогда

$$
Z(t)=W_{0}(t) Z(0)-\int_{0}^{t} W_{0}(t-s)\left[\dot{\mathrm{v}}(s) \cdot \nabla_{\mathrm{v}} F_{\mathrm{v}(s)}(s)\right] d s,
$$

где $W_{0}(t)$ - динамическая группа свободного уравнения Клейна-Гордона. Чтобы получить асимптотику (3.3), достаточно доказать, что $Z(t)=W_{0}(t) \boldsymbol{\Psi}_{+}+$ $r_{+}(t)$ с некоторым $\boldsymbol{\Psi}_{+} \in \mathscr{F}$ и $\left\|r_{+}(t)\right\|_{\mathscr{F}}=\mathscr{O}\left(t^{-1 / 2}\right)$. Это равносильно тому, что

$$
W_{0}(-t) Z(t)=\Psi_{+}+r_{+}^{\prime}(t),
$$

где $\left\|r_{+}^{\prime}(t)\right\|_{\mathscr{F}}=\mathscr{O}\left(t^{-1 / 2}\right)$, поскольку $W_{0}(t)$ - унитарная группа в пространстве Соболева $\mathscr{F}$ в силу закона сохранения энергии для свободного уравнения Клейна-Гордона. Наконец, (8.6) выполнено, поскольку из (8.5) следует, что

$$
W_{0}(-t) Z(t)=Z(0)-\int_{0}^{t} W_{0}(-s) R(s) d s, \quad R(s)=\dot{\mathrm{v}}(s) \cdot \nabla_{\mathrm{v}} F_{\mathrm{v}(s)}(s),
$$

где интеграл сходится в гильбертовом пространстве $\mathscr{F}$ со скоростью $\mathscr{O}\left(t^{-1 / 2}\right)$. Последнее верно, поскольку $\left\|W_{0}(-s) R(s)\right\|_{\mathscr{F}}=\mathscr{O}\left(s^{-3 / 2}\right)$ ввиду унитарности $W_{0}(-s)$ и скорости убывания $\|R(s)\|_{\mathscr{F}}=\mathscr{O}\left(s^{-3 / 2}\right)$. Обоснуем такую скорость убывания. Достаточно доказать, что $|\dot{\mathrm{v}}(s)|=\mathscr{O}\left(s^{-3 / 2}\right)$ или, что равносильно, $|\dot{p}(s)|=\mathscr{O}\left(s^{-3 / 2}\right)$. Подставляя (5.2) в последнее уравнение $(1.1)$, получим

$$
\begin{aligned}
\dot{p}(t)= & \int\left[\psi_{v(t)}(x-b(t))+\Psi(x-b(t), t)\right] \nabla \rho(x-b(t)-Q(t)) d x \\
= & \int \psi_{v(t)}(y) \nabla \rho(y) d y+\int \psi_{v(t)}(y)[\nabla \rho(y-Q(t))-\nabla \rho(y)] d y \\
& +\int \Psi(y, t) \nabla \rho(y-Q(t)) d y .
\end{aligned}
$$

Первый интеграл в правой части равен 0 в силу стационарных уравнений (3.2). Второй интеграл имеет порядок $\mathscr{O}\left(t^{-3 / 2}\right)$, поскольку $Q(t)=\mathscr{O}\left(t^{-3 / 2}\right)$ и в силу условия (2.8) на $\rho$. Наконец, третий интеграл имеет порядок $\mathscr{O}\left(t^{-3 / 2}\right)$ в силу оценки (6.9). Теорема доказана.

Для волнового уравнения доказательство аналогично, с соответствующей заменой показателей убывания. В случае уравнения Максвелла получаем уравнение

$$
\dot{Z}(t)=\mathscr{A} Z(t)-\dot{\mathrm{v}} \cdot \nabla_{\mathrm{v}} F_{\mathrm{v}(t)}(t), \quad \mathscr{A}(E, A)=(-\Delta A,-E),
$$

где

$$
\begin{gathered}
Z(t)=F(t)-F_{\mathrm{v}(\mathrm{t})}(t), \quad F(t)=(E(x, t), A(x, t)), \\
F_{\mathrm{v}(\mathrm{t})}(t)=\left(E_{\mathrm{v}(\mathrm{t})}(x-q(t)), A_{\mathrm{v}(\mathrm{t})}(x-q(t))\right), \quad \mathrm{v}(t):=\dot{q}(t) .
\end{gathered}
$$

Дальнейшие рассуждения аналогичны. 


\section{9. Убывание для линеаризованной динамики}

В оставшихся разделах мы докажем предложение 6.5, что завершит доказательство основного результата (теоремы 3.3). Обсудим общую стратегию доказательства предложения. Применим преобразование Фурье-Лапласа

$$
\Lambda X=\tilde{X}(\lambda)=\int_{0}^{\infty} e^{-\lambda t} X(t) d t, \quad \operatorname{Re} \lambda>0,
$$

к (6.12). В соответствии с предложением 6.5, мы ожидаем, что решение $X(t)$ ограничено по норме $\|\cdot\|_{-\beta}$. Тогда интеграл (9.1) сходится и является аналитической функцией в области $\operatorname{Re} \lambda>0$, и $\|\widetilde{X}(\lambda)\|_{-\beta} \leqslant C / \operatorname{Re} \lambda, \operatorname{Re} \lambda>0$. Выведем уравнение для $\widetilde{X}(\lambda)$, которое равносильно задаче Коши для (6.12) с начальным условием $X(0)=X_{0} \in \mathscr{E}_{-\beta}$. Для простоты обозначений в оставшейся части работы будем писать $A$ и $v$ вместо $A_{1}$ и $v_{1}$. Применяя преобразование Фурье-Лапласа к (6.12), получаем уравнение

$$
\lambda \widetilde{X}(\lambda)=A \widetilde{X}(\lambda)+X_{0}, \quad \operatorname{Re} \lambda>0 .
$$

Подчеркнем, что (9.2) равносильно задаче Коши для $X(t) \in C_{\mathrm{b}}\left([0, \infty) ; \mathscr{E}_{-\beta}\right)$. Поэтому решение $X(t)$ задается в виде $\widetilde{X}(\lambda)=-(A-\lambda)^{-1} X_{0}, \operatorname{Re} \lambda>0$, если резольвента $R(\lambda)=(A-\lambda)^{-1}$ существует при $\operatorname{Re} \lambda>0$.

Стратегия доказательства убывания (6.9) следующая. Во-первых, мы построим резольвенту $R(\lambda)$ при $\operatorname{Re} \lambda>0$ и докажем, что она является непрерывным оператором на $\mathscr{E}$. Тогда функция $\widetilde{X}(\lambda)$ принадлежит $\mathscr{E}-\beta$ и является аналитической при $\operatorname{Re} \lambda>0$. Во-вторых, мы должны обосновать, что существует (единственная) функция $X(t) \in C\left([0, \infty) ; \mathscr{E}_{-\beta}\right)$, удовлетворяющая (9.1).

Аналитичность $\widetilde{X}(\lambda)$ и аргументы типа Пэли-Винера (см. [43]) обеспечива-

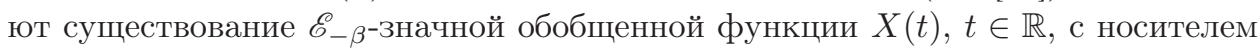
в $[0, \infty)$. Формально,

$$
\Lambda^{-1} \widetilde{X}=X(t)=\frac{1}{2 \pi} \int_{\mathbb{R}} e^{i \omega t} \tilde{X}(i \omega+0) d \omega, \quad t \in \mathbb{R} .
$$

Однако, чтобы проверить непрерывность $X(t)$ при $t \geqslant 0$, нам нужна дополнительная оценка для $\widetilde{X}(i \omega+0)$ при больших $|\omega|$. Наконец, для доказательства временно́го убывания $X(t)$ нам нужна дополнительная информация о гладкости и убывании $\widetilde{X}(i \omega+0)$. Точнее, нам следует доказать, что функция $\widetilde{X}(i \omega+0)$

i) гладкая вне точек $\omega=0$ и $\omega= \pm \mu$, где $\mu=\mu(v)>0$,

ii) имеет определенное убывание при $|\omega| \rightarrow \infty$,

iii) допускает разложение Пюизё в точках $\omega= \pm \mu$,

iv) аналитична при $\omega=0$, если $X_{0} \in \mathscr{Z}_{v}:=\mathbf{P}_{v} \mathscr{E}$ и $X_{0} \in \mathscr{E}_{\beta}$.

Тогда убывание (6.9) будет следовать из представления Фурье-Лапласа (9.3).

Мы подробно проверим свойства i)-iv) только для двух последних компонент $\widetilde{Q}(\lambda)$ и $\widetilde{P}(\lambda)$ вектора $\widetilde{X}(\lambda)=(\widetilde{\Psi}(\lambda), \widetilde{\Pi}(\lambda), \widetilde{Q}(\lambda), \widetilde{P}(\lambda))$. Эти свойства обеспечивают убывание (6.9) для векторных компонент $Q(t)$ и $P(t)$ решения $X(t)$. 
Однако мы не будем проверять i)-iv) для полевых компонент $\Psi(x, \lambda)$ и П $(x, \lambda)$. Мы выведем убывание (6.9) для полевых компонент непосредственно из неавтономных полевых уравнений системы (6.12), используя убывание компоненты $Q(t)$ и версию строгого принципа Гюйгенса для уравнения Клейна-Гордона.

\section{0. Построение резольвенты}

Мы строим резольвенту как ограниченный оператор на $\mathscr{E}$ при $\operatorname{Re} \lambda>0$. Для простоты обозначений будем писать $(\Psi(y), \Pi(y), Q, P)$ вместо $(\widetilde{\Psi}(y, \lambda), \widetilde{\Pi}(y, \lambda)$, $\widetilde{Q}(\lambda), \widetilde{P}(\lambda))$. Тогда $(9.2)$ имеет вид системы уравнений:

$$
\begin{aligned}
\Pi(y)+v \cdot \nabla \Psi(y)-\lambda \Psi(y) & =-\Psi_{0}(y), \\
\Delta \Psi(y)-m^{2} \Psi(y)+v \cdot \nabla \Pi(y)+Q \cdot \nabla \rho(y)-\lambda \Pi(y) & =-\Pi_{0}(y), \quad y \in \mathbb{R}^{3} . \\
B_{v} P-\lambda Q & =-Q_{0}, \\
-\langle\nabla \Psi(y), \rho(y)\rangle+\left\langle\nabla \psi_{v}(y), Q \cdot \nabla \rho(y)\right\rangle-\lambda P & =-P_{0},
\end{aligned}
$$

Шаг і). Рассмотрим первые два уравнения. В представлении Фурье они имеют вид

$$
\begin{aligned}
\widehat{\Pi}(k)-i v k \widehat{\Psi}(k)-\lambda \widehat{\Psi}(k) & =-\widehat{\Psi}_{0}(k), \\
\left(-k^{2}-m^{2}\right) \widehat{\Psi}(k)-(i v k+\lambda) \widehat{\Pi}(k) & =-\widehat{\Pi}_{0}(k)+i Q k \widehat{\rho}(k),
\end{aligned}
$$

$k \in \mathbb{R}^{3}$. Отсюда следует

$$
\begin{aligned}
& \widehat{\Psi}=\frac{1}{\widehat{D}}\left((i k v+\lambda) \widehat{\Psi}_{0}+\widehat{\Pi}_{0}-i k Q \widehat{\rho}\right), \\
& \widehat{\Pi}=\frac{1}{\widehat{D}}\left(-\left(k^{2}+m^{2}\right) \widehat{\Psi}_{0}+(i k v+\lambda) \widehat{\Pi}_{0}-i(i k v+\lambda) k Q \widehat{\rho}\right),
\end{aligned}
$$

где

$$
\widehat{D}=\widehat{D}(\lambda)=k^{2}+m^{2}+(i k v+\lambda)^{2} .
$$

Начиная с этого момента и далее мы работаем в такой системе координат в $x$-пространстве, в которой $v=(|v|, 0,0)$; тогда $v k=|v| k_{1}$. Подставяя выражение для $\widehat{\Psi}$ из (10.3) в четвертое уравнение системы $(10.1)$, получим

$$
\int d k i k\left((i k v+\lambda) \widehat{\Psi}_{0}+\Pi_{0}-i k Q \widehat{\rho}\right) \overline{\widehat{\rho}} / \widehat{D}+\int k \widehat{\psi_{v}} k Q \overline{\widehat{\rho}} d k-\lambda P=-P_{0} .
$$

С учетом формулы для $\widehat{\psi}_{v}$ (приложение $\mathrm{A}$ ) получаем $(K-H(\lambda)) Q+\lambda P=$ $P_{0}+\Phi(\lambda)$, где

$$
\Phi(\lambda)=\Phi\left(\Psi_{0}, \Pi_{0}\right)(\lambda):=i \int \frac{k}{\widehat{D}}\left((i k v+\lambda) \widehat{\Psi}_{0}+\widehat{\Pi}_{0}\right) \bar{\rho} d k .
$$

Здесь $K$ и $H(\lambda)-(3 \times 3)$-матрицы с матричными элементами

$$
K_{i j}=\int \frac{k_{i} k_{j}|\widehat{\rho}(k)|^{2} d k}{k^{2}+m^{2}-\left(|v| k_{1}\right)^{2}}, \quad H_{i j}(\lambda)=\int \frac{k_{i} k_{j}|\widehat{\rho}(k)|^{2} d k}{k^{2}+m^{2}+\left(i|v| k_{1}+\lambda\right)^{2}} .
$$


Матрица $K$ диагональна и положительно определена, поскольку $\widehat{\rho}(k)$ сферически симметрична и не равна тождественно нулю в силу (1.9). Матрица $H$ заведомо определена при $\operatorname{Re} \lambda>0$, поскольку знаменатель не обращается в нуль, и тоже диагональна. Заметим, что

$$
\Phi\left(\Psi_{0}, \Pi_{0}\right)(\lambda)=\Lambda\left\langle W^{1}(t)\left(\Psi_{0}, \Pi_{0}\right), \nabla \rho\right\rangle,
$$

где $\Lambda$ - преобразование Лапласа по $t, W^{1}(t)$ - первая компонента динамической группы $W(t)$, определенной ниже формулой (13.5). Наконец, третье и четвертое уравнения системы (10.1) преобразуются в уравнение

$$
M(\lambda)\left(\begin{array}{l}
Q \\
P
\end{array}\right)=\left(\begin{array}{c}
Q_{0} \\
P_{0}+\Phi
\end{array}\right), \quad \text { где } \quad M(\lambda)=\left(\begin{array}{cc}
\lambda E & -B_{v} \\
K-H(\lambda) & \lambda E
\end{array}\right) .
$$

Лемма 10.1. Матрица $M(\lambda)$ обратима при $\operatorname{Re} \lambda>0$.

Доказательство леммы см. в п. 11.1. Тогда

$$
\left(\begin{array}{l}
Q \\
P
\end{array}\right)=M^{-1}(\lambda)\left(\begin{array}{c}
Q_{0} \\
P_{0}+\Phi
\end{array}\right), \quad \operatorname{Re} \lambda>0
$$

Окончательно, формула (10.9) и формулы (10.3) определяют резольвенту $R(\lambda)=(A-\lambda)^{-1}, \operatorname{Re} \lambda>0$, в пространстве Фурье.

Шаг іi). Перейдем к $x$-представлению. Обращая матрицу системы (10.2), получим

$$
\left(\begin{array}{cc}
-(i v k+\lambda) & 1 \\
-\left(k^{2}+m^{2}\right) & -(i v k+\lambda)
\end{array}\right)^{-1}=\left[(i v k+\lambda)^{2}+k^{2}+m^{2}\right]^{-1}\left(\begin{array}{cc}
-(i v k+\lambda) & -1 \\
k^{2}+m^{2} & -(i v k+\lambda)
\end{array}\right) .
$$

Выполнив обратное преобразование Фурье, получим соответствующее фундаментальное решение

$$
G_{\lambda}(y)=\left(\begin{array}{cc}
v \cdot \nabla-\lambda & -1 \\
-\Delta+m^{2} & v \cdot \nabla-\lambda
\end{array}\right) g_{\lambda}(y)
$$

где $g_{\lambda}(y)$ - единственное фундаментальное решение умеренного роста для детерминанта

$$
D=D(\lambda)=-\Delta+m^{2}+(-v \cdot \nabla+\lambda)^{2}
$$

Таким образом,

$g_{\lambda}(y)=F_{k \rightarrow y}^{-1}\left[k^{2}+m^{2}+(i v k+\lambda)^{2}\right]^{-1}=F_{k \rightarrow y}^{-1}\left[k^{2}+m^{2}+\left(i|v| k_{1}+\lambda\right)^{2}\right]^{-1}, \quad y \in \mathbb{R}^{3}$.

Заметим, что знаменатель не обращается в нуль при $\operatorname{Re} \lambda>0$. Отсюда вытекает следующая лемма.

Лемма 10.2. Оператор $G_{\lambda}$ с интегральным ядром $G_{\lambda}\left(y-y^{\prime}\right)$ является непрерьвным оператором $H^{1}\left(\mathbb{R}^{3}\right) \oplus L^{2}\left(\mathbb{R}^{3}\right) \rightarrow H^{2}\left(\mathbb{R}^{3}\right) \oplus H^{1}\left(\mathbb{R}^{3}\right)$ при $\operatorname{Re} \lambda>0$. 
Таким образом, из (10.2) и (10.10) мы получаем сверточное представление

$$
\begin{aligned}
& \Psi=-(v \cdot \nabla-\lambda) g_{\lambda} * \Psi_{0}+g_{\lambda} * \Pi_{0}+\left(g_{\lambda} * \nabla \rho\right) \cdot Q, \\
& \Pi=-\left(-\Delta+m^{2}\right) g_{\lambda} * \Psi_{0}-(v \cdot \nabla-\lambda) g_{\lambda} * \Pi_{0}-(v \cdot \nabla-\lambda)\left(g_{\lambda} * \nabla \rho\right) \cdot Q .
\end{aligned}
$$

Шаг iii). Вычислим $g_{\lambda}(y)$. Для $v=(|v|, 0,0)$ с $|v|<1$ фундаментальное решение имеет вид

$$
\begin{array}{cl}
g_{\lambda}(y)=\frac{e^{-\kappa|\widetilde{y}|-\kappa_{1} \widetilde{y}_{1}}}{4 \pi|\widetilde{y}|}, \quad \widetilde{y}:=\left(\gamma y_{1}, y_{2}, y_{3}\right), \quad \kappa:=\gamma \sqrt{\lambda^{2}+\mu^{2}}, \\
\kappa_{1}:=\gamma|v| \lambda, \quad \gamma=\frac{1}{\sqrt{1-v^{2}}}, \quad \mu:=\frac{m}{\gamma},
\end{array}
$$

где мы выбираем $\operatorname{Re} \kappa>0$ при $\operatorname{Re} \lambda>0$. Заметим, что

$$
0<\operatorname{Re} \kappa_{1}<\operatorname{Re} \kappa \quad \text { при } \operatorname{Re} \lambda>0 \text {. }
$$

Это неравенство следует из экспоненциального убывания фундаментального решения, которое вытекает из свойств аналитичности при помощи аргументов типа Пэли-Винера, поскольку квадратичная форма $\widehat{D}(k)$ не обращается в нуль в некоторой комплексной окрестности вещественного пространства $\mathbb{R}^{3}$ при $\operatorname{Re} \lambda>0$. Результат этих рассуждений сформулируем в виде следующей леммы.

Лемма 10.3. i) Onepamop $D=D(\lambda)$ обратим в $L^{2}\left(\mathbb{R}^{3}\right)$ nри $\operatorname{Re} \lambda>0$, а его фундаментальное решение (10.14) экспоненииально убъвает по у.

ii) Из формул (10.14) следует, что для любого фиксированного у функиия Грина $g_{\lambda}(y)$ допускает аналитическое продолюсение по $\lambda$ на риманову поверхность алгебрачческой функиии $\sqrt{\lambda^{2}+\mu^{2}}$ с точками ветвления $\lambda= \pm i \mu$.

iii) Матричная функиия $M(\lambda)$ (соответственно $\left.M^{-1}(\lambda)\right)$ допускает аналитическое (соответственно мероморфное) продолжение на риманову поверхность функиии $\sqrt{\lambda^{2}+\mu^{2}}$.

Аналитическое продолжение функции $M(\lambda)$ существует в силу леммы $10.3, \mathrm{ii})$ и определений (10.6), поскольку согласно $(2.8)$ функция $\rho(x)$ имеет компактный носитель. Тогда обратная матрица мероморфна, так как она существует для больших $\operatorname{Re} \lambda$. Последнее вытекает из (10.8), поскольку $H(\lambda) \rightarrow 0$ при $\operatorname{Re} \lambda \rightarrow \infty$, согласно (10.6).

Для волнового уравнения все вычисления этого раздела сохраняются с подстановкой $m=0$. Фундаментальное решение (10.14) имеет вид

$$
g_{\lambda}(y)=\frac{e^{-\kappa|\widetilde{y}|-\kappa_{1} \widetilde{y}_{1}}}{4 \pi|\widetilde{y}|}, \quad \widetilde{y}:=\left(\gamma y_{1}, y_{2}, y_{3}\right), \quad \kappa=\gamma \lambda, \quad \kappa_{1}=\gamma|v| \lambda
$$

В этом случае лемма 10.3 трансформируется следующим образом.

Лемма 10.4. i) Onератор $D=D(\lambda)$ обратим в $L^{2}\left(\mathbb{R}^{3}\right)$ nри $\operatorname{Re} \lambda>0$, а его фундаментальное решение (10.14) экспоненциально убъвает по у. 
ii) Из формул (10.16) следует, что для любого фиксированного у функция Грина $g_{\lambda}(y)$ допускает аналитическое продолжение по $\lambda$ на всю комплексную плоскость $\mathbb{C}$.

iii) Матричная функция $M(\lambda)$ (соответственно $\left.M^{-1}(\lambda)\right)$ допускает аналитическое (соответственно мероморфное) продолжение на всю комплексную плоскость $\mathbb{C}$.

Для уравнения Максвелла вычисление резольвенты аналогично, однако имеет более сложную алгебраическую структуру. Приведем основные результаты. Уравнение (6.12) имеет вид

$$
\begin{gathered}
\dot{e}=v \cdot \nabla e-\Delta a+\Pi_{s}\left(r \cdot \nabla \rho v-\rho B_{v}(\pi-\langle\rho, a\rangle)\right), \quad \dot{a}=-e+v \cdot \nabla a, \\
\dot{r}=B_{v}(\pi-\langle\rho, a\rangle), \quad \dot{\pi}=\langle\rho, \nabla(v \cdot a)\rangle-\left\langle r \cdot \nabla \rho, \nabla\left(v \cdot A_{v}\right)\right\rangle .
\end{gathered}
$$

Чтобы упростить его, выполним замену переменных $\varphi=\pi-\langle\rho, a\rangle$. Тогда $\pi=$ $\varphi+\langle\rho, a\rangle$. Если будет доказано убывание $\varphi$ и $a$, то и $\pi$ будет иметь аналогичное убывание. Далее, $\dot{\varphi}=\dot{\pi}-\langle\rho, \dot{a}\rangle=\dot{\pi}-\langle\rho,-e+v \cdot \nabla a\rangle=\langle\rho, e\rangle+\langle\rho, \nabla(v \cdot a)-$ $(v \cdot \nabla) a\rangle-\left\langle r \cdot \nabla \rho, \nabla\left(v \cdot A_{v}\right)\right\rangle$ согласно последнему уравнению системы (10.17). Тогда система (10.17) равносильна следующей системе:

$$
\begin{gathered}
\dot{e}=v \cdot \nabla e-\Delta a+\Pi_{s}\left(r \cdot \nabla \rho v-\rho B_{v} \varphi\right), \quad \dot{a}=-e+v \cdot \nabla a, \\
\dot{r}=B_{v} \varphi, \quad \dot{\varphi}=\langle\rho, e\rangle+\langle\rho, v \wedge(\nabla \wedge a)\rangle-\left\langle r \cdot \nabla \rho, \nabla\left(v \cdot A_{v}\right)\right\rangle .
\end{gathered}
$$

При выводе последнего уравнения мы применили тождество $\nabla(v \cdot a)-(v \cdot \nabla) a=$ $v \wedge(\nabla \wedge a)$. Обозначим той же буквой $A$ оператор

$$
A\left(\begin{array}{c}
e \\
a \\
r \\
\varphi
\end{array}\right):=\left(\begin{array}{c}
v \cdot \nabla e-\Delta a+\Pi\left(r \cdot \nabla \rho v-\rho B_{v} \varphi\right) \\
-e+v \cdot \nabla a \\
B_{v} \varphi \\
\langle\rho, e\rangle+\langle\rho, v \wedge(\nabla \wedge a)\rangle-\left\langle r \cdot \nabla \rho, \nabla\left(v \cdot A_{v}\right)\right\rangle
\end{array}\right) .
$$

Будет доказано убывание решения $X=(e, a, r, \varphi)$ уравнения $\dot{X}(t)=A X(t)$. Для этого мы строим и изучаем резольвенту оператора $A$. После преобразования Лапласа (сохраняя старые обозначения $(e(y), a(y), r, \varphi)$ для преобразованных функций) мы приходим к системе

$$
\begin{gathered}
v \cdot \nabla e-\Delta a+\Pi_{s}\left(r \cdot \nabla \rho v-\rho B_{v} \varphi\right)-\lambda e=-e_{0}, \quad-e+v \cdot \nabla a-\lambda a=-a_{0}, \\
B_{v} \varphi-\lambda r=-r_{0}, \quad\langle\rho, e\rangle+\langle\rho, v \wedge(\nabla \wedge a)\rangle-\left\langle r \cdot \nabla \rho, \nabla\left(v \cdot A_{v}\right)\right\rangle-\lambda \varphi=-\varphi_{0} .
\end{gathered}
$$

Применим к ней преобразование Фурье и из первых двух уравнений получим

$$
\begin{gathered}
\widehat{a}=\frac{1}{\widehat{D}}\left((\lambda+i k v) \widehat{a}_{0}-\widehat{e}_{0}+\widehat{\Pi}\right), \quad \widehat{\Pi}:=\widehat{\rho} \widehat{\Pi}\left(i(k r) v+B_{v} \varphi\right), \\
\widehat{e}=\frac{k^{2} \widehat{a}_{0}+(\lambda+i k v) \widehat{e}_{0}-(\lambda+i k v) \widehat{\Pi}}{\widehat{D}} \\
\widehat{D}=\widehat{D}(\lambda)=k^{2}+(\lambda+i k v)^{2},
\end{gathered}
$$


$\widehat{D}(\lambda) \neq 0$ при $\operatorname{Re} \lambda>0$. Четвертое уравнение системы $(10.20)$ имеет вид $\langle\rho, e\rangle+$ $\langle\rho, v \wedge(\nabla \wedge a)\rangle-\left\langle r \cdot \nabla \rho, \nabla\left(v \cdot A_{v}\right)\right\rangle-\lambda \varphi=-\varphi_{0}$. С этого момента и далее мы работаем в такой системе координат в $x$-пространстве, в которой $v=(|v|, 0,0)$, а значит, $v k=|v| k_{1}$. В силу (10.21) прямым вычислением получаем $\langle\rho, e\rangle=$ $\Phi-C_{1} r+F_{1} \varphi$, где

$$
\Phi=\Phi\left(\lambda, e_{0}, a_{0}\right):=\int \frac{\left(k^{2} \widehat{a}_{0}+(\lambda+i k v) \widehat{e}_{0}\right) \overline{\hat{\rho}}}{\widehat{D}} d k
$$

и $C_{1}=\operatorname{diag}\left(c_{11}(\lambda), c_{12}(\lambda), c_{13}(\lambda)\right), F_{1}=\operatorname{diag}\left(f_{11}(\lambda), f_{12}(\lambda), f_{13}(\lambda)\right)$ (здесь и далее через $\operatorname{diag}(a, b, c)$ мы обозначаем диагональную матрицу с элементами $a, b, c$ на главной диагонали), причем

$$
\begin{aligned}
& c_{11}(\lambda)=i|v| \int \frac{k_{1}\left(\lambda+i k_{1}|v|\right)|\hat{\rho}|^{2}}{\widehat{D}(\lambda)}\left(1-\frac{k_{1}^{2}}{k^{2}}\right) d k, \\
& c_{1 j}(\lambda)=-i|v| \int \frac{k_{1} k_{j}^{2}\left(\lambda+i k_{1}|v|\right)|\widehat{\rho}|^{2}}{k^{2} \widehat{D}(\lambda)} d k, \quad j=2,3, \\
& f_{11}(\lambda)=\nu^{3} \int \frac{\left(\lambda+i k_{1}|v|\right)|\widehat{\rho}|^{2}}{\widehat{D}(\lambda)}\left(\frac{k_{1}^{2}}{k^{2}}-1\right) d k, \\
& f_{1 j}(\lambda)=\nu \int \frac{\left(\lambda+i k_{1}|v|\right) \mid \widehat{\rho}^{2}}{\widehat{D}(\lambda)}\left(\frac{k_{j}^{2}}{k^{2}}-1\right) d k, \quad j=2,3 ;
\end{aligned}
$$

здесь $\nu=\sqrt{1-v^{2}}$. Очевидно, что $c_{12}=c_{13}$. Кроме того, $c_{11}+c_{12}+c_{13}=0$, поэтому $c_{11}=-c_{12}-c_{13}=-2 c_{12}$. Матрица $C_{1}(\lambda)$ упрощается и приобретает вид $C_{1}(\lambda)=\operatorname{diag}\left(c_{1}(\lambda), c_{12}(\lambda), c_{12}(\lambda)\right)$,

$$
c_{1}(\lambda):=-2 c_{12}(\lambda), \quad c_{12}(\lambda)=-\frac{i|v|}{2} \int \frac{k_{1}\left(\lambda+i k_{1}|v|\right)|\widehat{\rho}|^{2}}{\widehat{D}(\lambda)}\left(1-\frac{k_{1}^{2}}{k^{2}}\right) d k .
$$

Аналогично, $f_{12}=f_{13}$ и

$$
f_{12}(\lambda)=-\frac{\nu}{2} \int \frac{\left(\lambda+i k_{1}|v|\right)|\widehat{\rho}|^{2}}{\widehat{D}(\lambda)}\left(1+\frac{k_{1}^{2}}{k^{2}}\right) d k .
$$

Далее, $\langle\rho, v \wedge(\nabla \wedge a)\rangle=\Psi-C_{2} r+F_{2} \varphi$, где

$$
\Psi=\Psi\left(\lambda, e_{0}, a_{0}\right):=\int d k v \wedge\left(-i k \wedge \frac{(\lambda+i k v) \widehat{a}_{0}-\widehat{e}_{0}}{\widehat{D}(\lambda)}\right) \bar{\rho}
$$

и $C_{2}(\lambda)=\operatorname{diag}\left(0, c_{22}(\lambda), c_{23}(\lambda)\right), F_{2}(\lambda)=\operatorname{diag}\left(0, f_{22}(\lambda), f_{23}(\lambda)\right)$, где

$$
c_{2 j}(\lambda)=-v^{2} \int d k \frac{|\widehat{\rho}|^{2} k_{j}^{2}}{\widehat{D}(\lambda)}, \quad f_{2 j}(\lambda)=i \nu|v| \int d k \frac{|\widehat{\rho}|^{2} k_{1}}{\widehat{D}(\lambda)}, \quad j=2,3 .
$$

Заметим, что

$$
\Phi(\lambda)=\Lambda\left\langle W^{1}(t)\left(e_{0}, a_{0}\right), \rho\right\rangle,
$$


где $W^{1}(t)$ - первая компонента динамической группы $W(t)$, которая определена ниже формулой (13.15), где положено П $(t) \equiv 0$. Аналогично,

$$
\Psi(\lambda)=\Lambda\left\langle v \wedge\left(\nabla \wedge W^{2}(t)\left(e_{0}, a_{0}\right)\right), \rho\right\rangle,
$$

где $W^{2}(t)$ - вторая компонента этой группы. Далее, $c_{22}=c_{23}, f_{22}=f_{23}$ и

$$
c_{22}(\lambda)=-\frac{v^{2}}{2} \int d k \frac{|\widehat{\rho}|^{2}\left(k_{2}^{2}+k_{3}^{2}\right)}{\widehat{D}(\lambda)}=-\frac{v^{2}}{2} \int d k \frac{|\widehat{\rho}|^{2}\left(k^{2}-k_{1}^{2}\right)}{\widehat{D}(\lambda)} .
$$

Наконец, $\left\langle r \cdot \nabla \rho, \nabla\left(v \cdot A_{v}\right)\right\rangle=G r$, где $G=\operatorname{diag}\left(g_{1}, g_{2}, g_{3}\right)$,

$$
g_{j}=v^{2} \int \frac{\left(k^{2}-k_{1}^{2}\right) k_{j}^{2} \mid \widehat{\rho}^{2}}{k^{2}\left(k^{2}-k_{1}^{2} v^{2}\right)} d k, \quad j=1,2,3 .
$$

При этом $g_{2}=g_{3}$ и мы обозначим $g:=g_{2}=g_{3}$. Положим $C(\lambda)=C_{1}(\lambda)+C_{2}(\lambda)$, $F(\lambda)=F_{1}(\lambda)+F_{2}(\lambda)$. Более подробно, $C(\lambda)=\operatorname{diag}\left(c_{1}(\lambda), c(\lambda), c(\lambda)\right), F(\lambda)=$ $\operatorname{diag}\left(f_{1}(\lambda), f(\lambda), f(\lambda)\right)$,

$$
c(\lambda):=c_{12}(\lambda)+c_{22}(\lambda), \quad f_{1}(\lambda):=f_{11}(\lambda), \quad f(\lambda):=f_{12}(\lambda)+f_{22}(\lambda) .
$$

Итак, четвертое уравнение принимает вид $(C(\lambda)+G) r+(\lambda E-F(\lambda)) \varphi=\varphi_{0}+$ $\Phi(\lambda)+\Psi(\lambda)$. Запишем его вместе с третьим уравнением системы (10.20) в виде

$$
M(\lambda)\left(\begin{array}{l}
r \\
\varphi
\end{array}\right)=\left(\begin{array}{c}
r_{0} \\
\varphi_{0}+\Phi(\lambda)+\Psi(\lambda)
\end{array}\right), \quad \text { где } M(\lambda)=\left(\begin{array}{cc}
\lambda E & -B_{v} \\
C(\lambda)+G & \lambda E-F(\lambda)
\end{array}\right) .
$$

Матрица $M(\lambda)$ обратима при $\operatorname{Re} \lambda>0$. Тогда

$$
\left(\begin{array}{l}
r \\
\varphi
\end{array}\right)=M^{-1}(\lambda)\left(\begin{array}{c}
r_{0} \\
\varphi_{0}+\Phi(\lambda)+\Psi(\lambda)
\end{array}\right), \quad \operatorname{Re} \lambda>0 .
$$

Формулы (10.21) и (10.36) выражают резольвенту $R(\lambda)=(A-\lambda)^{-1}, \operatorname{Re} \lambda>0$, в представлении Фурье.

Лемма 10.5. i) Оператор $D(\lambda)$, определенный в пространстве Фуръе как оператор умножения на символ (10.22), обратим в $L^{2}\left(\mathbb{R}^{3}\right)$ nри $\operatorname{Re} \lambda>0$, и его фундаментальное решение $g_{\lambda}(y)$ экспоненциально убъвает при $|y| \rightarrow \infty$.

ii) Распределение $g_{\lambda}(\cdot)$ допускает аналитическое продолжение по $\lambda$ из области $\operatorname{Re} \lambda>0$ на всю комплексную плоскость $\mathbb{C}$.

iii) Матричная функция $M(\lambda)$ (соответственно $M^{-1}(\lambda)$ ) допускает аналитическое (соответственно мероморфное) продолжение по параметру $\lambda$ из области $\operatorname{Re} \lambda>0$ на всю комплексную плоскость.

\section{1. Свойства резольвенты}

\section{1. Аналитичность в правой полуплоскости.}

ПРеДЛОЖЕНИЕ 11.1. Операторнозначная функция $R(\lambda): \mathscr{E} \rightarrow \mathscr{E}$ аналитична при $\operatorname{Re} \lambda>0$. 
ДокАзАтельство. Достаточно доказать, что оператор $A-\lambda: \mathscr{E} \rightarrow \mathscr{E}$ имеет ограниченный обратный оператор при $\operatorname{Re} \lambda>0$. Напомним, что $A=A_{v, v}$, где $|v|<1$.

Шаг i). Покажем, что $\operatorname{Ker}(A-\lambda)=0$ при $\operatorname{Re} \lambda>0$. Допустим, что $X_{\lambda}=\left(\Psi_{\lambda}, \Pi_{\lambda}, Q_{\lambda}, P_{\lambda}\right) \in \mathscr{E}$ удовлетворяет соотношению $(A-\lambda) X_{\lambda}=0$, т. е. $X_{\lambda}-$ решение $(10.1)$ с $\Psi_{0}=\Pi_{0}=0$ и $Q_{0}=P_{0}=0$. Надо доказать, что $X_{\lambda}=0$. Сначала проверим, что $P_{\lambda}=0$. Действительно, траектория $X:=X_{\lambda} e^{\lambda t} \in C(\mathbb{R}, \mathscr{E})$ является решением уравнения $\dot{X}=A X$ или уравнения (5.13) с $w=v$. Тогда $\mathscr{H}_{v, v}(X(t))$ экспоненциально растет в силу (5.18), поскольку матрица $B_{v}$ положительна. Но такой рост противоречит закону сохранения для $\mathscr{H}_{v, v}$, следующему из леммы $5.2, \mathrm{ii})$, поскольку $X(t) \in C^{1}(\mathbb{R}, \mathscr{E})$. Это последнее включение вытекает из леммы 10.2 , так как $\left(\Psi_{\lambda}, \Pi_{\lambda}\right)$ удовлетворяют уравнениям (10.13) с $\Psi_{0}=\Pi_{0}=0$ и $Q=Q_{\lambda}$. Теперь $\lambda Q_{\lambda}=B_{v} P_{\lambda}=0$ в силу третьего уравнения (10.1), поэтому $Q_{\lambda}=0$, так как $\lambda \neq 0$. Наконец, $\Psi_{\lambda}=0, \Pi_{\lambda}=0$ ввиду уравнений (10.13) с $Q=Q_{\lambda}=0$.

Шаг іi). Имеем $A-\lambda=A_{0}+T$, где

$$
\begin{aligned}
A_{0} & =\left(\begin{array}{cccc}
v \cdot \nabla-\lambda & 1 & 0 & 0 \\
\Delta-m^{2} & v \cdot \nabla-\lambda & 0 & 0 \\
0 & 0 & -\lambda & 0 \\
0 & 0 & 0 & -\lambda
\end{array}\right), \\
T & =\left(\begin{array}{cccc}
0 & 0 & 0 & 0 \\
0 & 0 & \cdot \nabla \rho & 0 \\
0 & 0 & 0 & B_{v} \\
\langle\cdot, \nabla \rho\rangle & 0 & \left\langle\nabla \psi_{v}, \cdot \nabla \rho\right\rangle & 0
\end{array}\right) .
\end{aligned}
$$

Оператор $T$ - конечномерный, а оператор $A_{0}^{-1}$ ограничен на $\mathscr{E}$ по лемме 10.2 . Наконец, $A-\lambda=A_{0}\left(I+A_{0}^{-1} T\right)$, где $A_{0}^{-1} T-$ компактный оператор. Поскольку мы знаем, что $\operatorname{Ker}\left(I+A_{0}^{-1} T\right)=0$, оператор $\left(I+A_{0}^{-1} T\right)$ обратим согласно теории Фредгольма. Предложение доказано.

Заметим, что из доказательства следует обратимость матрицы $M(\lambda)$ при $\operatorname{Re} \lambda>0$.

Для волнового уравнения и уравнения Максвелла лемма и доказательство сохраняются с соответствующей заменой фазовых пространств.

11.2. Регулярность на мнимой оси. Следующий шаг - исследование предельных значений резольвенты $R(\lambda)$ на мнимой оси $\lambda=i \omega, \omega \in \mathbb{R}$, которое необходимо для доказательства убывания (6.9) решения $X(t)=(\Psi(t), \Pi(t)$, $Q(t), P(t))$. Сначала опишем непрерывный спектр оператора $A=A_{v, v}$ на мнимой оси. По определению, непрерывный спектр состоит из таких $\omega \in \mathbb{R}$, для которых резольвента $R(i \omega+0)$ не является ограниченным оператором на $\mathscr{E}$. Согласно формулам (10.13), это происходит в случае, когда функция Грина $g_{\lambda}\left(y-y^{\prime}\right)$ теряет экспоненциальное убывание. Это равносильно условию $\operatorname{Re} \kappa=0$, где $\kappa$ задано формулой (10.14): $\kappa=\gamma \sqrt{\mu^{2}-\omega^{2}}$. Таким образом, $i \omega$ принадлежит непрерывному спектру, если $|\omega| \geqslant \mu=m \sqrt{1-v^{2}}$. По лемме 10.3, 
предельная матрица

$$
M(i \omega):=M(i \omega+0)=\left(\begin{array}{cc}
i \omega E & -B_{v} \\
K-H(i \omega+0) & i \omega E
\end{array}\right), \quad \omega \in \mathbb{R},
$$

существует, и ее элементы являются непрерывными функциями от $\omega \in \mathbb{R}$, гладкими при $|\omega|<\mu$ и $|\omega|>\mu$. Напомним, что точка $\lambda=0$ принадлежит дискретному спектру оператора $A$ по лемме $5.4, \mathrm{i})$, поэтому $M(i \omega+0)$ может также быть необратимой при $\omega=0$.

ПРЕДЛОЖЕНИЕ 11.2. Пусть выполнены условия (2.8) и (1.9), a $|v|<1$. Тогда предельная матрица $M(i \omega+0)$ обратима при $\omega \neq 0, \omega \in \mathbb{R}$.

Мы рассмотрим отдельно три случая: $0<|\omega|<\mu,|\omega|=\mu$ и $|\omega|>\mu$. Напомним, что матрицы $K$ и $H$ диагональны с элементами $K_{j j}, H_{j j}$, определенными в (10.6), и $H_{22}=H_{33}$. Поскольку $v=(|v|, 0,0)$, матрица $B_{v}$ также диагональна: $B_{v}:=\nu(E-v \otimes v)=\operatorname{diag}\left(\nu^{3}, \nu, \nu\right)$. Обозначим $F(\omega):=-K+H(i \omega+0)$; эта матрица тоже диагональна; пусть $f_{1}:=F_{11}(\omega)$ и $f=F_{22}(\omega)=F_{33}(\omega)$. Тогда по (11.1)

$$
\operatorname{det} M(i \omega)=\operatorname{det}\left(\begin{array}{cc}
i \omega E & -B_{v} \\
-F(\omega) & i \omega E
\end{array}\right)=-\left(\omega^{2}+\nu^{3} f_{1}\right)\left(\omega^{2}+\nu f\right)^{2}, \quad \omega \in \mathbb{R} .
$$

I. Сначала рассмотрим случай $0<|\omega|<\mu$. Тогда обратимость матрицы $M(i \omega)$ следует из (11.2) в силу следующей леммы.

ЛЕмма 11.3. При $0<|\omega|<\mu$ матрища $F(\omega)$ положительно определена, m.e. $F_{j j}(\omega)>0, j=1,2,3$.

Доказательство проводится прямым вычислением (см. подробности в [25; лемма 15.2].

II. $\omega= \pm \mu$. Рассмотрим, например, $\omega=\mu$. В этом случае

$$
H_{j j}(i \mu)=\int \frac{k_{j}^{2}|\widehat{\rho}(k)|^{2} d k}{k_{2}^{2}+k_{3}^{2}+\left(\nu k_{1}-m|v|\right)^{2}} .
$$

Подынтегральное выражение имеет единственную особую точку. Особенность интегрируемая, поэтому $F_{j j}(\mu)$ также положительны. Значит, матрица $M(i \mu)$ также обратима.

III. $|\omega|>\mu$. В этом случае требуется другое обоснование. Обратимость матрицы $M(i \omega)$ будет следовать из (11.2) в силу следующей леммы, доказательство которой опирается на аргументы из [44] (глава VII, формула (58)).

Лемма 11.4. Если выполнено условие (1.9) и $|\omega|>\mu$, то мнимая часть матрицы $(\omega /|\omega|) F(\omega)$ отрицательно определена, т.е. $(\omega /|\omega|) \operatorname{Im} F_{j j}(\omega)<0$, $j=1,2,3$.

Поскольку $F(\omega)=-K+H(i \omega+0)$, где матрица $K$ вещественна, рассмотрим только матрицу $H(i \omega+0)$. Для $\varepsilon>0$ имеем

$$
H_{j j}(i \omega+\varepsilon)=\int \frac{k_{j}^{2}|\widehat{\rho}(k)|^{2} d k}{k_{1}^{2}+k_{2}^{2}+k_{3}^{2}-\left(|v| k_{1}+\omega-i \varepsilon\right)^{2}+m^{2}}, \quad j=1,2,3 .
$$


Рассмотрим знаменатель $\widehat{D}(i \omega+\varepsilon, k)=k^{2}+m^{2}-\left(|v| k_{1}+\omega-i \varepsilon\right)^{2}$. Выше было показано, что $\widehat{D}(i \omega, k) \neq 0$, если $|\omega|<\mu$, и что $\widehat{D}(i \omega, k)$ обращается в нуль в одной точке, если $|\omega|=\mu$. Однако $\widehat{D}(i \omega, k)=0$ на эллипсоиде $T_{\omega}$ при $|\omega|>\mu$, где

$$
T_{\omega}=\left\{k:\left(\nu k_{1}-\frac{|v| \omega}{\nu}\right)^{2}+k_{2}^{2}+k_{3}^{2}=R^{2}:=\frac{\omega^{2}-\mu^{2}}{\nu^{2}}\right\},
$$

здесь $\nu=\sqrt{1-v^{2}}$. Тогда из формулы Сохоцкого-Племеля для $C^{1}$-функций следует, что

$$
\operatorname{Im} H_{j j}(i \omega+0)=-\frac{\omega}{|\omega|} \pi \int_{T_{\omega}} \frac{k_{j}^{2}|\widehat{\rho}(k)|^{2}}{|\nabla \widehat{D}(i \omega, k)|} d S,
$$

где $d S$ - элемент площади поверхности. Отсюда немедленно следует утверждение леммы, поскольку подынтегральное выражение в (11.4) положительно в силу условия Винера (1.9).

Обоснование формулы (11.4) приведено в [25; лемма 15.2]. Лемма 11.4 и предложение 11.2 доказаны.

СЛЕДСТВИЕ 11.5. Из предложения 11.2 следует, что матрица $M^{-1}(i \omega)$ является гладкой функиией от $\omega \in \mathbb{R}$ за исключением трех точек $\omega=0, \pm \mu$.

Доказательство леммы 11.4 - единственное место в работе, где необходимо условие Винера. В лемме 11.3 мы пользуемся только тем, что плотность $\rho(x)$ не равна тождественно нулю.

В случае волнового уравнения $\mu=0$. Это значит, что крайние точки непрерывного спектра сливаются в точке $\lambda=0$, совпадая при этом с точкой 0 дискретного спектра. Продолжение обратной матрицы $M^{-1}(\lambda)$ на мнимую ось делается только при помощи рассуждения с формулой Сохоцкого-Племеля. Лемма 11.4 сохраняется с подстановкой $\mu=0$ и $m=0$.

В случае уравнения Максвелла также имеем совпадение крайних точек непрерывного спектра с точкой 0 дискретного спектра, рассуждения аналогичны.

11.3. Особые точки спектра. Напомним, что формула (10.36) задает преобразование Фурье--Лапласа $\widetilde{Q}(\lambda), \widetilde{P}(\lambda)$. Тогда векторные компоненты выражаются интегралом Фурье

$$
\left(\begin{array}{l}
Q(t) \\
P(t)
\end{array}\right)=\frac{1}{2 \pi} \int e^{i \omega t} M^{-1}(i \omega)\left(\begin{array}{c}
Q_{0} \\
P_{0}+\Phi(i \omega)
\end{array}\right) d \omega,
$$

если он сходится в смысле обобщенных функций. Одного следствия 11.5 недостаточно для доказательства сходимости интеграла и его убывания. Нужна дополнительная информация о регулярности матрицы $M^{-1}(i \omega)$ в особых точках $\omega=0, \pm \mu$, а также подходящие оценки при $|\omega| \rightarrow \infty$. Рассмотрим все эти случаи по отдельности.

Уравнение Клейна-Гордона. I. Сначала рассмотрим точки $\pm \mu$. 
Лемма 11.6. Матрица $M^{-1}(i \omega)$ допускает следующее разложение Пюизё в окрестности точек $\pm \mu$ : существует $\varepsilon_{ \pm}>0$ такое, что

$$
M^{-1}(i \omega)=\sum_{k=0}^{\infty} R_{k}^{ \pm}(\omega \mp \mu)^{k / 2}, \quad|\omega \mp \mu|<\varepsilon_{ \pm}, \quad \omega \in \mathbb{R} .
$$

Достаточно построить аналогичное разложение для $M(i \omega)$. Тогда (11.6) выполняется также и для $M^{-1}(i \omega)$, поскольку матрицы $M( \pm i \mu)$ обратимы. Асимптотика для $M(i \omega)$ выполнена ввиду сверточного представления:

$$
H_{i j}(\lambda)=\left\langle g_{\lambda} * \partial_{j} \rho, \partial_{i} \rho\right\rangle
$$

поскольку $g_{\lambda}$ допускает соответствующее разложение Пюизё ввиду явной формулы (10.14).

II. Во-вторых, мы изучим асимптотическое поведение матрицы $M^{-1}(\lambda)$ на бесконечности. Напомним, что матрица $M^{-1}(\lambda)$ была первоначально определена при $\operatorname{Re} \lambda>0$, но допускает мероморфное продолжение на риманову поверхность функции $\sqrt{\lambda^{2}+\mu^{2}}$ (см. лемму 10.3). Следующее предложение является частным случаем общей теоремы об оценке усеченной резольвенты на непрерывном спектре. Эта оценка играет решающую роль при изучении долговременной асимптотики общих линейных гиперболических уравнений (см. [44]).

ПРЕДЛОЖЕНИЕ 11.7. Существуют $(6 \times 6)$-матрица $R_{0}$ и матричная функиия $R_{1}(\omega)$ maкuе, что

$$
\begin{gathered}
M^{-1}(i \omega)=\frac{R_{0}}{\omega}+R_{1}(\omega), \\
\left|\partial_{\omega}^{k} R_{1}(\omega)\right| \leqslant \frac{C_{k}}{|\omega|^{2}}, \quad \omega \in \mathbb{R}, \quad|\omega| \geqslant \mu+1, \quad k=0,1,2, \ldots .
\end{gathered}
$$

Ввиду структуры (11.1) матрицы $M(i \omega)$, достаточно доказать следующую оценку для элементов матрицы $H(i \omega):=H(i \omega+0)$ :

$$
\left|\partial_{\omega}^{k} H_{j j}(i \omega)\right| \leqslant \frac{C_{k}}{|\omega|}, \quad \omega \in \mathbb{R}, \quad|\omega| \geqslant \mu+1, \quad j=1,2,3 .
$$

Перепишем (11.7) в виде $H_{i j}(\lambda)=\left\langle D^{-1}(\lambda) \partial_{j} \rho, \partial_{i} \rho\right\rangle$, $\operatorname{Re} \lambda>0$, где $D(\lambda)$ - оператор $(10.11)$, а $D^{-1}(\lambda)$ - ограниченный оператор в $L^{2}\left(\mathbb{R}^{3}\right)$. Обозначим через $B_{R}$ шар $\left\{x \in \mathbb{R}^{3}:|x|<R\right\}$. Оценка (11.9) следует из более общей оценки

$$
\left\|\partial_{\omega}^{k} D^{-1}(i \omega+0) f\right\|_{L^{2}\left(B_{R}\right)} \leqslant \frac{C_{k}(R)}{|\omega|}\|f\|_{L^{2}\left(B_{R}\right)}, \quad \omega \in \mathbb{R}, \quad|\omega| \geqslant \mu+1,
$$

которая выполняется для каждого $R>0$ и всех функций $f(y) \in L_{R}^{2}:=\{f(y) \in$ $\left.L^{2}\left(\mathbb{R}^{3}\right): \operatorname{supp} f \subset B_{R}\right\}$. А именно, (11.9) следует из (11.10), примененного к функции $f(y)=\partial_{j} \rho(y) \in L_{R}^{2}$ с $R \geqslant R_{\rho}$ (см. (2.8)). Оценка (11.10) следует из общей оценки [45; теорема 3] (см. также [46; оценка $($ А.2')], [47; теорема 8.1], [48; теорема 3]). 
III. Наконец, мы рассмотрим точку $\omega=0$ с наибольшей сингулярностью. Эта точка является изолированным полюсом конечного порядка по лемме 10.3, поэтому имеет место разложение Лорана для матрицы $L(\omega):=M^{-1}(i \omega)$. Мы можем найти точный вид этого разложения, т. е. вычислить векторные компоненты

$$
\left(\begin{array}{l}
\widetilde{Q}(i \omega) \\
\widetilde{P}(i \omega)
\end{array}\right)=L(\omega)\left(\begin{array}{c}
Q_{0} \\
P_{0}+\Phi(i \omega)
\end{array}\right) .
$$

Лемма 11.8. і) Векторные компоненты имеют вид

$$
\begin{aligned}
& \widetilde{Q}(i \omega)=\frac{1}{\omega} L_{11}(\omega) Q_{0}+\frac{1}{\omega^{2}} L_{12}(\omega)\left(P_{0}+\Phi(i \omega)\right), \\
& \widetilde{P}(i \omega)=L_{21}(\omega) Q_{0}+\frac{1}{\omega} L_{22}(\omega)\left(P_{0}+\Phi(i \omega)\right),
\end{aligned}
$$

где $L_{i j}(\omega), i, j=1,2,-$ гладкие в окрестности нуля диагональные матриць размера $3 \times 3, L_{i j}(\omega) \in C^{\infty}\left(-\varepsilon_{0}, \varepsilon_{0}\right)$ при достаточно малом $\varepsilon_{0}$.

ii) Имеет место равенство

$$
L_{11}=i L_{12} B_{v}^{-1} \text {. }
$$

Для доказательства леммы достаточно выявить следующую структуру обратной матрицы $L(\omega)$ :

$$
L(\omega)=\left(\begin{array}{cc}
\frac{1}{\omega} L_{11} & \frac{1}{\omega^{2}} L_{12} \\
L_{21} & \frac{1}{\omega} L_{22}
\end{array}\right),
$$

это сделано в приложении В. Проиллюстрируем основную идею доказательства убывания векторных компонент $(Q(t), P(t))$ - устранение полюсов в (11.11) при помощи условий симплектической ортогональности. Строгое доказательство приведено в следующем разделе. Во-первых, заметим, что

$$
P_{0}+\Phi(i \omega) \sim P_{0}+\Phi(0)+\Phi^{\prime}(0) i \omega, \quad \omega \rightarrow 0 .
$$

Тогда, ввиду следующей леммы, устраняется полюс первого порядка в $\widetilde{P}(i \omega)$.

Лемма 11.9. і) Условия симплектической ортогоналъности $\Omega\left(X_{0}, \tau_{j}\right)=0$, $j=1,2,3$, имеют вид

$$
P_{0}+\Phi(0)=0 \text {. }
$$

ii) Условия симплектической ортогональности $\Omega\left(X_{0}, \tau_{j+3}\right)=0, j=1,2,3$, имеют вид

$$
B_{v}^{-1} Q_{0}+\Phi^{\prime}(0)=0 .
$$

Далее, ввиду (11.14) и (11.15),

$$
\begin{aligned}
\frac{1}{\omega} L_{11}(\omega) Q_{0}+\frac{1}{\omega^{2}} L_{12}(\omega)\left(P_{0}+\Phi(i \omega)\right) & \sim \frac{1}{\omega} L_{11}(\omega) Q_{0}+\frac{1}{\omega^{2}} L_{12}(\omega) \Phi^{\prime}(0) i \omega \\
=\frac{1}{\omega}\left(L_{11}(\omega) Q_{0}+i L_{12}(\omega) \Phi^{\prime}(0)\right) & =\frac{1}{\omega}\left(i L_{12}(\omega) B_{v}^{-1} Q_{0}+i L_{12}(\omega) \Phi^{\prime}(0)\right) \\
= & \frac{i L_{12}(\omega)}{\omega}\left(B_{v}^{-1} Q_{0}+\Phi^{\prime}(0)\right), \quad \omega \rightarrow 0 .
\end{aligned}
$$


Тем самым, по предыдущей лемме, устраняются оба полюса первого и второго порядка в (11.11).

ДокАЗАТЕЛЬСтво ЛЕмМЫ 11.9. Напомним, что

$$
\Phi(0)=i \int d k \frac{\left(i k v \widehat{\Psi}_{0}+\Pi_{0}\right) k \widehat{\widehat{\rho}}}{\widehat{D}}, \quad \widehat{D}=k^{2}+m^{2}-(k v)^{2} .
$$

С другой стороны, для $j=1,2,3$ имеем

$$
\begin{aligned}
0 & =\Omega\left(X_{0}, \tau_{j}\right)=-\left\langle\Psi_{0}, \partial_{j} \pi_{v}\right\rangle+\left\langle\Pi_{0}, \partial_{j} \psi_{v}\right\rangle-P_{0} \cdot e_{j} \\
& =\int d k \frac{\widehat{\Psi}_{0}(k v) \bar{\rho} k_{j}}{\widehat{D}}-i \int d k \frac{\widehat{\Pi}_{0} \widehat{\widehat{\rho}} k_{j}}{\widehat{D}}-\left(P_{0}\right)_{j}=-\left(\Phi(0)+P_{0}\right)_{j} .
\end{aligned}
$$

Таким образом, $j$-я компонента вектора $\Phi(0)+P_{0}$ равна 0 при $j=1,2,3$. Далее,

$$
\begin{aligned}
0=\Omega\left(X_{0}, \tau_{j+3}\right)=i \int d k \frac{\widehat{\Psi}_{0} k_{j}\left(k^{2}+m^{2}+(k v)^{2}\right) \overline{\widehat{\rho}}}{\widehat{D}^{2}} \\
+\int d k \frac{2 \widehat{\Pi}_{0}(k v) k_{j} \overline{\widehat{\rho}}}{\widehat{D}^{2}}+Q_{0} \cdot \partial_{v_{j}} p_{v}, \quad j=1,2,3 .
\end{aligned}
$$

Заметим, что $Q_{0} \cdot \partial_{v_{j}} p_{v}=Q_{0} \cdot B_{v}^{-1} e_{j}=B_{v}^{-1} Q_{0} \cdot e_{j}$. Тогда эти условия симплектической ортогональности совпадают с $\Phi^{\prime}(0)+B_{v}^{-1} Q_{0}=0$, см. ниже формулу (12.8). Лемма доказана.

Для волнового уравнения и уравнения Максвелла имеют место только случаи II и III. Предложение 11.7 принимает следующий вид.

Лемма 11.10. Существуют матрица $R_{0}$ и матричная бункция $R_{1}(\omega)$ такие, что

$$
\begin{gathered}
M^{-1}(i \omega)=\frac{R_{0}}{\omega}+R_{1}(\omega), \quad\left|\partial_{\omega}^{k} R_{1}(\omega)\right| \leqslant \frac{C_{k}}{|\omega|^{2}}, \\
\omega \in \mathbb{R}, \quad|\omega|>r>0, \quad k=0,1,2, \ldots,
\end{gathered}
$$

при достаточно большом $r$.

Леммы 11.8, 11.9 справедливы и в случае волнового уравнения, т. е. при $m=0,-$ соответственно изменяются матричные элементы.

Для уравнения Максвелла соответствующие утверждения имеют следующий вид.

Лемма 11.11. і) Матрица $M^{-1}(i \omega)$ имеет вид

$$
M^{-1}(i \omega)=\left(\begin{array}{cc}
\frac{1}{\omega} L_{11} & \frac{1}{\omega^{2}} L_{12} \\
L_{21} & \frac{1}{\omega} L_{22}
\end{array}\right),
$$

где $L_{i j}(\omega), i, j=1,2,-$ гладкие в окрестности нуля диагональные $(3 \times 3)$-матрицы, $L_{i j}(\omega) \in C^{\infty}\left(-\varepsilon_{0}, \varepsilon_{0}\right)$ при достаточно малом $\varepsilon_{0}$. Кроме того,

$$
L_{11}=i L_{12} B_{v}^{-1}+i L_{3},
$$


где $L_{3}$ - диагональная $(3 \times 3)$-матрища, $L_{3}(\omega) \in C^{\infty}\left(-\varepsilon_{0}, \varepsilon_{0}\right)$ (см. приложение $\mathrm{B})$.

ii) Векторные компоненты имеют вид

$$
\begin{aligned}
& \widetilde{r}(i \omega)=\frac{1}{\omega} L_{11}(\omega) r_{0}+\frac{1}{\omega^{2}} L_{12}(\omega)\left(\varphi_{0}+\Phi(i \omega)+\Psi(i \omega)\right), \\
& \widetilde{\varphi}(i \omega)=L_{21}(\omega) r_{0}+\frac{1}{\omega} L_{22}(\omega)\left(\varphi_{0}+\Phi(i \omega)+\Psi(i \omega)\right) .
\end{aligned}
$$

iii) Условия симплектической ортогональности имеют вид

$$
\varphi_{0}+\Phi(0)+\Psi(0)=0, \quad\left(B_{v}^{-1}+L_{12}^{-1}(0) L_{3}(0)\right) r_{0}+\Phi^{\prime}(0)+\Psi^{\prime}(0)=0 .
$$

\section{2. Временно́е убывание векторных компонент}

Докажем убывание (6.9) для компонент $Q(t)$ и $P(t)$.

Лемма 12.1. Пусть $X_{0} \in \mathscr{Z}_{v, \beta}$. Тогда $Q(t), P(t)$ непрерывны и

$$
|Q(t)|+|P(t)| \leqslant \frac{C\left(\rho, \bar{v}, d_{0}\right)}{(1+|t|)^{3 / 2}}, \quad t \geqslant 0 .
$$

Из предыдущего рассмотрения особых точек спектра следует сходимость интеграла Фурье (11.5) в смысле обобщенных функций к непрерывной функции от $t \geqslant 0$. Докажем (12.1). Сначала заметим, что из условия $X_{0} \in \mathscr{Z}_{v, \beta}$ следует, что вся траектория $X(t)$ лежит в $\mathscr{Z}_{v, \beta}$, поскольку пространство $\mathscr{Z}_{v, \beta}$ инвариантно относительно генератора $A_{v, v}$. Для $X_{0}$, не принадлежащих $\mathscr{Z}_{v, \beta}$, компоненты $Q(t)$ и $P(t)$ могут содержать неубывающие члены, которые соответствуют особой точке $\omega=0$. Действительно, мы знаем, что линеаризованная динамика допускает неубывающие секулярные решения (см. (6.13)), имеющие соответствующие векторные компоненты. Мы покажем, что условия симплектической ортогональности обеспечивают (12.1). Разложим интеграл Фурье (11.5) на три слагаемых при помощи разбиения единицы $\zeta_{1}(\omega)+\zeta_{2}(\omega)+\zeta_{3}(\omega)=1, \omega \in \mathbb{R}$ :

$$
\begin{aligned}
\left(\begin{array}{l}
Q(t) \\
P(t)
\end{array}\right) & =\frac{1}{2 \pi} \int e^{i \omega t}\left(\zeta_{1}(\omega)+\zeta_{2}(\omega)+\zeta_{3}(\omega)\right) M^{-1}(i \omega)\left(\begin{array}{c}
Q_{0} \\
P_{0}+\Phi(i \omega)
\end{array}\right) d \omega \\
& =I_{1}(t)+I_{2}(t)+I_{3}(t),
\end{aligned}
$$

где функции $\zeta_{k}(\omega) \in C^{\infty}(\mathbb{R})$ имеют следующие носители:

$$
\begin{gathered}
\operatorname{supp} \zeta_{1} \subset\left\{\omega \in \mathbb{R}: \varepsilon_{0} / 2<|\omega|<\mu+2\right\}, \quad \operatorname{supp} \zeta_{2} \subset\{\omega \in \mathbb{R}:|\omega|>\mu+1\}, \\
\operatorname{supp} \zeta_{3} \subset\left\{\omega \in \mathbb{R}:|\omega|<\varepsilon_{0}\right\} .
\end{gathered}
$$

Тогда функции $I_{k}(t)$ обладают следующими свойствами:

i) функция $I_{1}(t)$ убывает как $(1+|t|)^{-3 / 2}$ в силу разложения Пюизё (11.6);

ii) функция $I_{2}(t)$ убывает как $(1+|t|)^{-3 / 2}$ ввиду предложения 11.7. 
Здесь мы не используем условия симплектической ортогональности. Ввиду (11.8) и (10.7) получаем:

$$
\begin{aligned}
I_{2}(t) & =\frac{1}{2 \pi} \int e^{i \omega t} \zeta_{2}(\omega)\left[\frac{R_{0}}{\omega}\left(\begin{array}{c}
Q_{0} \\
P_{0}
\end{array}\right)+\frac{R_{0}}{\omega}\left(\begin{array}{c}
0 \\
\Phi(i \omega)
\end{array}\right)+R_{1}\left(\begin{array}{c}
Q_{0} \\
P_{0}
\end{array}\right)+R_{1}\left(\begin{array}{c}
0 \\
\Phi(i \omega)
\end{array}\right)\right] \\
& =s_{1}(t)\left(\begin{array}{l}
Q_{0} \\
P_{0}
\end{array}\right)+s_{2}(t)\left(\begin{array}{c}
Q_{0} \\
P_{0}
\end{array}\right)+s_{1}(t) *\left(\begin{array}{c}
0 \\
f(t)
\end{array}\right)+s_{2}(t) *\left(\begin{array}{c}
0 \\
f(t)
\end{array}\right)
\end{aligned}
$$

где (см. (9.3)) $s_{1}(t):=\Lambda^{-1} \zeta_{2} R_{0} / \omega, s_{2}(t)=\Lambda^{-1} \zeta_{2} R_{1}(\omega)$ и

$$
f(t):=\Lambda^{-1} \Phi(i \omega)=\left\langle W^{1}(t)\left(\Psi_{0}, \Pi_{0}\right), \nabla \rho\right\rangle,
$$

поскольку $\Phi$ определяется формулами (10.5) и (10.7). Далее, в силу леммы 13.2 имеет место убывание

$$
|f(t)| \leqslant C(1+t)^{-3 / 2}
$$

Ввиду (12.6) все члены в (12.4) являются непрерывными при $t \geqslant 0$ и убывают как $t^{-3 / 2}$, поскольку $s_{1}(t), s_{2}(t)$ непрерывны при $t \geqslant 0$, ограничены и убывают как $t^{-\infty}$ при $t \rightarrow \infty$.

iii) Наконец, функция $I_{3}(t)$ убывает как $t^{-\infty}$, если $\left(\Psi_{0}, \Pi_{0}, Q_{0}, P_{0}\right) \in \mathscr{Z}_{v}$, ввиду следующего утверждения.

Лемма 12.2. При $\left(\Psi_{0}, \Pi_{0}, Q_{0}, P_{0}\right) \in \mathscr{Z}_{v}$ векторные компоненты

$$
\left(\begin{array}{l}
\widetilde{Q}(i \omega) \\
\widetilde{P}(i \omega)
\end{array}\right)=M^{-1}(i \omega)\left(\begin{array}{c}
Q_{0} \\
P_{0}+\Phi(i \omega)
\end{array}\right)
$$

как функции от $\omega$ принадлежат пространству $C^{\infty}\left(-\varepsilon_{0} ; \varepsilon_{0}\right)$ при достаточно малом $\varepsilon_{0}$.

i) Рассмотрим сначала компоненту $\widetilde{P}(i \omega)$. Имеем $\widetilde{P}(i \omega)=L_{21} Q_{0}+L_{22}\left(P_{0}+\right.$ $\Phi(i \omega)) / \omega$, где

$$
\Phi(i \omega)=i \int d k \frac{i\left(k_{1} v+\omega\right) \widehat{\Psi}_{0}(k)+\widehat{\Pi}_{0}(k)}{m^{2}+k^{2}-\left(v k_{1}+\omega\right)^{2}} k \overline{\widehat{\rho}},
$$

а условия симплектической ортогональности для начальных данных по лемме 11.9 имеют вид $P_{0}+\Phi(0)=0$. Тогда

$$
P_{0}+\Phi(i \omega)=\Phi(i \omega)-\Phi(0)=i \omega \Sigma_{1}(\omega)
$$

где $\Sigma_{1}(\omega) \in C^{\infty}\left(-\varepsilon_{0} ; \varepsilon_{0}\right)$ ввиду явного представления

$$
\Sigma_{1}(\omega)=\int d k \frac{\omega\left(i k_{1} v \widehat{\Psi}_{0}(k)+\widehat{\Pi}_{0}(k)\right)+i\left(m^{2}+k^{2}+\left(k_{1} v\right)^{2}\right) \widehat{\Psi}_{0}(k)+2 v k_{1} \widehat{\Pi}_{0}(k)}{\left(m^{2}+k^{2}-\left(v k_{1}+\omega\right)^{2}\right)\left(m^{2}+k^{2}-\left(v k_{1}\right)^{2}\right)} k \overline{\widehat{\rho}} .
$$

Таким образом, $\widetilde{P}(i \omega)=L_{21}(\omega) Q_{0}+L_{22}(\omega) \Sigma_{1}(\omega) \in C^{\infty}\left(-\varepsilon_{0} ; \varepsilon_{0}\right)$. 
ii) Переходим к $Q(i \omega)$. Имеем

$$
\begin{aligned}
\widetilde{Q}(i \omega) & =\frac{1}{\omega} L_{11} Q_{0}+\frac{1}{\omega^{2}} L_{12}\left(P_{0}+\Phi(i \omega)\right)=\frac{L_{12}}{\omega}\left(i B_{v}^{-1} Q_{0}+\frac{P_{0}+\Phi(i \omega)}{\omega}\right) \\
& =\frac{L_{12}}{\omega}\left(i B_{v}^{-1} Q_{0}+\frac{\Phi(i \omega)-\Phi(0)}{\omega}\right)=\frac{i L_{12}}{\omega}\left(i B_{v}^{-1} Q_{0}+\Sigma_{1}(\omega)\right)
\end{aligned}
$$

согласно (12.7). Далее, поскольку $\Sigma_{1}(0)=\Phi^{\prime}(0)$, по лемме 11.9 условия симплектической ортогональности $\Omega\left(X_{0}, \tau_{j+3}\right)=0, j=1,2,3$, имеют вид $\Sigma_{1}(0)+$ $B_{v}^{-1} Q_{0}=0$. Тогда $B_{v}^{-1} Q_{0}+\Sigma_{1}(\omega)=\Sigma_{1}(\omega)-\Sigma_{1}(0)=\omega \Sigma_{2}(\omega)$, где

$\Sigma_{2}(\omega)=\int d k \frac{i n^{2}\left(\omega+3 v k_{1}\right) \widehat{\Psi}_{0}(k)+\left(n^{2}+2 v k_{1}\left(\omega+2 v k_{1}\right)\right) \widehat{\Pi}_{0}(k)}{n^{4}\left(n^{2}-\omega\left(\omega+2 k_{1}|v|\right)\right)} k \widehat{\rho} \in C^{\infty}\left(-\varepsilon_{0} ; \varepsilon_{0}\right)$,

здесь $n^{2}:=k^{2}+m^{2}-\left(k_{1}|v|\right)^{2}$. В итоге $\widetilde{Q}(i \omega)=i L_{12}(\omega) \Sigma_{2}(\omega) \in C^{\infty}\left(-\varepsilon_{0} ; \varepsilon_{0}\right)$. Лемма доказана.

В случае волнового уравнения справедлива следующая теорема.

TeOpema 12.3. Пусть

$$
X_{0}=\mathbf{P}_{v} Z_{0}, \quad Z_{0} \in \mathscr{E}_{\beta}, \quad \beta=4+\delta .
$$

Тогда $Q(t), P(t)$ непреръьнъь $и$

$$
|Q(t)|+|P(t)| \leqslant \frac{C\left(\rho, \bar{v}, d_{\beta}\right)}{(1+|t|)^{1+\delta}}\left\|X_{0}\right\|_{\beta}, \quad t \geqslant 0 .
$$

Обозначим $X_{0}=\left(\Psi_{0}, \Pi_{0}, Q_{0}, P_{0}\right)=\mathbf{P}_{v} Z_{0}$, где $Z_{0}=\left(\Psi_{0}^{\prime}, \Pi_{0}^{\prime}, Q_{0}^{\prime}, P_{0}^{\prime}\right) \in \mathscr{E}_{4+\delta}$. Разложим интеграл Фурье (11.5) на два слагаемых при помощи разбиения единицы $\zeta_{1}(\omega)+\zeta_{2}(\omega)=1, \omega \in \mathbb{R}$ :

$$
\left(\begin{array}{l}
Q(t) \\
P(t)
\end{array}\right)=\frac{1}{2 \pi} \int e^{i \omega t}\left(\zeta_{1}(\omega)+\zeta_{2}(\omega)\right)\left(\begin{array}{l}
\widetilde{Q}(i \omega) \\
\widetilde{P}(i \omega)
\end{array}\right) d \omega=\left(\begin{array}{l}
Q_{1}(t) \\
P_{1}(t)
\end{array}\right)+\left(\begin{array}{l}
Q_{2}(t) \\
P_{2}(t)
\end{array}\right)
$$

где функции $\zeta_{k}(\omega) \in C^{\infty}(\mathbb{R})$ имеют носители

$$
\operatorname{supp} \zeta_{1} \subset\left\{\omega \in \mathbb{R}:|\omega|<\varepsilon_{0}\right\}, \quad \operatorname{supp} \zeta_{2} \subset\left\{\omega \in \mathbb{R}:|\omega|>\frac{\varepsilon_{0}}{2}\right\} .
$$

i) Используем предложение 11.7, чтобы доказать, что функции $Q_{2}(t), P_{2}(t)$ непрерывны и

$$
\left|Q_{2}(t)\right|+\left|P_{2}(t)\right| \leqslant C(1+|t|)^{-3-\delta}\left\|X_{0}\right\|_{\beta} .
$$

Эта часть доказательства аналогична случаю Клейна-Гордона, единственное существенное отличие - другой порядок убывания $f(t)$.

Лемма 12.4. Имеет место следующее убывание:

$$
|f(t)| \leqslant C(1+t)^{-3-\delta}\left\|X_{0}\right\|_{\beta} .
$$


Во-первых, из (6.14) следует, что $X_{0}=\mathbf{P}_{v} Z_{0}=Z_{0}-\boldsymbol{\Pi}_{v} Z_{0}$. Тогда $\Psi_{0}=$ $\Psi_{0}^{\prime}+\Psi_{0}^{\prime \prime}, \Pi_{0}=\Pi_{0}^{\prime}+\Pi_{0}^{\prime \prime}$, где

$$
\Psi_{0}^{\prime \prime}=\sum_{j=1}^{3}\left(\lambda_{j} \partial_{j} \psi_{v}-\mu_{j} \partial_{v_{j}} \psi_{v}\right), \quad \Pi_{0}^{\prime \prime}=\sum_{j=1}^{3}\left(\lambda_{j} \partial_{j} \pi_{v}-\mu_{j} \partial_{v_{j}} \pi_{v}\right),
$$

$\lambda_{j}=\lambda_{j}\left(v, Z_{0}\right), \mu_{j}=\mu_{j}\left(v, Z_{0}\right)$. Из (10.7) получаем $\Phi\left(\Psi_{0}^{\prime}+\Psi_{0}^{\prime \prime}, \Pi_{0}^{\prime}+\Pi_{0}^{\prime \prime}\right)(i \omega)=$ $\widetilde{f}_{1}(i \omega)+\widetilde{f}_{2}(i \omega)$, где $\widetilde{f}_{j}(i \omega)=\Lambda_{t \rightarrow i \omega} f_{j}(t), j=1,2$, и

$$
f_{1}(t):=\left\langle W^{1}(t)\left(\Psi_{0}^{\prime}, \Pi_{0}^{\prime}\right), \nabla \rho\right\rangle, \quad f_{2}(t):=\left\langle W^{1}(t)\left(\Psi_{0}^{\prime \prime}, \Pi_{0}^{\prime \prime}\right), \nabla \rho\right\rangle .
$$

Заметим, что

$$
\left|f_{1}(t)\right| \leqslant C(1+t)^{-3-\delta}
$$

в силу (13.10) с $\alpha=3+\delta$. Для $f_{2}(t)$ мы выведем убывание

$$
\left|f_{2}(t)\right| \leqslant C(1+|t|)^{-4} \text {. }
$$

Оно получается, если переписать $f_{2}(t)$ в виде осциллирующего интеграла

$$
\left\langle F_{x \rightarrow k} W^{1}\left(\Psi_{0}^{\prime \prime}, \Pi_{0}^{\prime \prime}\right),-i k \widehat{\rho}(k)\right\rangle,
$$

где подынтегральная функция имеет порядок $\mathscr{O}(|k|)$ при $k \rightarrow 0$ ввиду условия (1.10) и условий регулярности (2.8). Из (12.16) и (12.17) следует убывание (12.14).

ii) Теперь докажем убывание для компонент $Q_{1}(t)$ и $P_{1}(t)$. Эта часть доказательства существенно использует условия симплектической ортогональности и отличается от случая Клейна-Гордона.

Лемма 12.5. Справедливы следующие оценки:

$$
\left|P_{1}(t)\right| \leqslant C(1+t)^{-2-\delta}\left\|X_{0}\right\|_{\beta}, \quad\left|Q_{1}(t)\right| \leqslant C(1+t)^{-1-\delta}\left\|X_{0}\right\|_{\beta}, \quad t \geqslant 0 .
$$

Сначала докажем (12.18) для $P_{1}(t)$ на основании представления (11.11). А именно,

$$
P_{1}(t)=\Lambda^{-1} \zeta_{1}(\omega) L_{21}(\omega) Q_{0}+\Lambda^{-1} \zeta_{1}(\omega) L_{22}(\omega) \frac{P_{0}+\Phi(i \omega)}{\omega}=P_{1}^{\prime}(t)+P_{1}^{\prime \prime}(t) .
$$

Очевидно, что первое слагаемое $P^{\prime}(t)$ убывает как $t^{-\infty}$ по лемме 11.8 , ii). Второе слагаемое запишем в виде свертки $P_{1}^{\prime \prime}(t)=\Lambda^{-1} \zeta_{1} L_{22} * g(t)$, где $g(t):=$ $\Lambda^{-1}\left[\left(P_{0}+\Phi(i \omega)\right) / \omega\right]$. Воспользовавшись условиями симплектической ортогональности (11.15), получим

$$
g(t)=\Lambda^{-1} \frac{\Phi(i \omega)-\Phi(0)}{\omega}=i \int_{\infty}^{t} f(s) d s
$$

Последнее равенство вытекает из следующего свойства преобразования Лапласа: если функция $f(t) \in L^{1}(0, \infty)$ ограничена и

$$
g(t)=\int_{\infty}^{t} f(s) d s, \quad \text { то } \Lambda g(\lambda)=\frac{\Lambda f(\lambda)-\Lambda f(0)}{\lambda} .
$$


Наконец, $g(t)$ убывает как $t^{-2-\delta}$ при $t \geqslant 0$ ввиду (12.6), а значит, $P_{1}^{\prime \prime}(t)$ убывает как $t^{-2-\delta}$ при $t \geqslant 0$.

Теперь докажем (12.18) для $Q_{1}(t)$. В силу (11.11), (11.12) и условий симплектической ортогональности (11.15),

$$
\begin{aligned}
\widetilde{Q}(i \omega) & =\frac{L_{12}}{\omega}\left(i B_{v}^{-1} Q_{0}+\frac{P_{0}+\Phi(i \omega)}{\omega}\right)=\frac{L_{12}}{\omega}\left(i B_{v}^{-1} Q_{0}+\frac{\Phi(i \omega)-\Phi(0)}{\omega}\right) \\
& =\frac{L_{12}}{\omega}\left(i B_{v}^{-1} Q_{0}+\widetilde{g}(\omega)\right)=L_{12} \frac{i B_{v}^{-1} Q_{0}+\widetilde{g}(0)+\widetilde{g}(i \omega)-\widetilde{g}(0)}{\omega} \\
& =L_{12} \frac{\widetilde{g}(i \omega)-\widetilde{g}(0)}{\omega}
\end{aligned}
$$

поскольку $i B_{v}^{-1} Q_{0}+\widetilde{g}(0)=0$ ввиду условий симплектической ортогональности (11.16) с учетом того, что $\widetilde{g}(0)=i \Phi^{\prime}(0)$. Тогда $Q_{1}(t)=\Lambda^{-1} \zeta_{1}(\omega) L_{12} * h(t)$ в силу (12.2), где

$$
h(t):=\Lambda^{-1} \frac{\widetilde{g}(i \omega)-\widetilde{g}(0)}{\omega}=i \int_{\infty}^{t} g(s) d s,
$$

опять согласно (12.19). Этот интеграл убывает как $t^{-1-\delta}$ при $t \geqslant 0$ ввиду (12.6), тогда $Q_{1}$ убывает как $t^{-1-\delta}$ при $t \geqslant 0$. Теорема 12.3 доказана.

В случае уравнений Максвелла теорема принимает следующий вид.

Tеорема 12.6. Пусть $X_{0}=\mathbf{P}_{v} Z_{0}, Z_{0} \in \mathscr{E}_{\beta}, \beta=4+\delta$. Тогда $r(t), \varphi(t)$ непрерывны и

$$
|r(t)|+|\varphi(t)| \leqslant \frac{C\left(\rho, \bar{v}, d_{\beta}\right)}{(1+|t|)^{1+\delta}}\left\|X_{0}\right\|_{\beta}, \quad t \geqslant 0 .
$$

Доказательство аналогично случаю волнового уравнения.

\section{3. Временно́е убывание полей}

Уравнение Клейна-Гордона. Докажем убывание полевых компонент $\Psi(x, t)$, $\Pi(x, t)$, соответствующее (6.9). Обозначим $F(t)=(\Psi(\cdot, t), \Pi(\cdot, t))$. Мы построим поля как решения первых двух уравнений системы (6.12), где $A_{1}$ задано формулой (5.4) с $w=v=v\left(t_{1}\right)$. Эти два уравнения имеют вид

$$
\dot{F}(t)=\left(\begin{array}{cc}
v \cdot \nabla & 1 \\
\Delta-m^{2} & v \cdot \nabla
\end{array}\right) F(t)+\left(\begin{array}{c}
0 \\
Q(t) \cdot \nabla \rho
\end{array}\right) .
$$

По лемме 12.1 мы знаем, что $Q(t)$ - непрерывная функция от $t \geqslant 0$, и

$$
|Q(t)| \leqslant \frac{C\left(\rho, \bar{v}, d_{0}\right)}{(1+|t|)^{3 / 2}}, \quad t \geqslant 0 .
$$

Значит, предложение 6.5 сводится к следующему утверждению. 
ПреДЛОЖение 13.1. і) Пусть $Q(t) \in C\left([0, \infty) ; \mathbb{R}^{3}\right)$ u $F_{0} \in \mathscr{F}$. Тогда уравнение (13.1) имеет единственное решение $F(t) \in C([0, \infty) ; \mathscr{F})$ с начальным условием $F(0)=F_{0}$.

ii) Если $F_{0} \in \mathscr{F}_{\beta}$ и имеет место убъвание (13.2), то соответствуюиие поля тоже убывают равномерно по $v$ :

$$
\|F(t)\|_{-\beta} \leqslant \frac{C\left(\rho, \bar{v}, d_{0},\left\|F_{0}\right\|_{\beta}\right)}{(1+|t|)^{3 / 2}}, \quad t \geqslant 0,
$$

при $|v| \leqslant \bar{v}$ слюбъм $\bar{v} \in(0,1)$.

ДокАзАтельство. Утверждение і) выведем из представления Дюамеля

$$
F(t)=W(t) F_{0}+\left[\int_{0}^{t} W(t-s)\left(\begin{array}{c}
0 \\
Q(s) \cdot \nabla \rho
\end{array}\right) d s\right], \quad t \geqslant 0,
$$

где $W(t)$ - динамическая группа модифицированного уравнения Клейна-Гордона

$$
\dot{F}(t)=\left(\begin{array}{cc}
v \cdot \nabla & 1 \\
\Delta-m^{2} & v \cdot \nabla
\end{array}\right) F(t) .
$$

Группу $W(t)$ можно выразить через группу $W_{0}(t)$ стандартного уравнения Клейна-Гордона

$$
\dot{\Phi}(t)=\left(\begin{array}{cc}
0 & 1 \\
\Delta-m^{2} & 0
\end{array}\right) \Phi(t) .
$$

А именно, задача (13.6) соответствует (13.5) с $v=0$, и легко видеть, что

$$
[W(t) F(0)](x)=\left[W_{0}(t) F(0)\right](x+v t), \quad x \in \mathbb{R}^{3}, \quad t \in \mathbb{R} .
$$

Обозначим через $W(x-y, t)$ и $W_{0}(x-y, t)$ интегральные матричные ядра операторов $W(t)$ и $W_{0}(t)$ соответственно. Тогда из (13.7) следует, что

$$
W(x-y, t)=W_{0}(x-y+v t, t), \quad x, y \in \mathbb{R}^{3}, \quad t \in \mathbb{R} .
$$

Из тождества (13.7) следует также закон сохранения энергии для группы $W(t)$. А именно, для $(\Psi(\cdot, t), \Pi(\cdot, t))=W(t) F(0)$ имеем

$$
\int\left(|\Pi(x, t)-v \cdot \nabla \Psi(x, t)|^{2}+|\nabla \Psi(x, t)|^{2}+m^{2}|\Psi(x, t)|^{2}\right) d x=\text { const, } \quad t \in \mathbb{R} .
$$

В частности, это дает

$$
\left\|W(t) F_{0}\right\|_{\mathscr{F}} \leqslant C(\bar{v})\left\|F_{0}\right\|_{\mathscr{F}}, \quad t \in \mathbb{R} .
$$

Из этой оценки и из (13.4) следует утверждение і). Докажем утверждение іi).

Лемма 13.2. Для любъх $\beta>3 / 2, \bar{v}<1$ u $F_{0} \in \mathscr{F}_{\beta}$ имеет место следующее убъвание:

$$
\left\|W(t) F_{0}\right\|_{-\beta} \leqslant \frac{C(\beta, \bar{v})}{(1+t)^{3 / 2}}\left\|F_{0}\right\|_{\beta}, \quad t \geqslant 0,
$$

где $W(t)$ - динамическая группа модифицированного уравнения Клейна-Гордона (13.5) $c|v|<\bar{v}$. 
Доказательство леммы можно дать при помощи общих методов Йенсена и Като [47], основанных на фундаментальной оценке Агмона [46; оценка $\left.\left(\mathrm{A} .2^{\prime}\right)\right]$. Короткое независимое доказательство имеется в [25; лемма 18.2]. Теперь утверждение іi) следует из леммы 13.2 и представления Дюгамеля (13.4). Действительно, $W(t) F_{0}$ убывает как $t^{-3 / 2}$ по лемме 13.2. Поэтому

$$
\begin{aligned}
\left\|\int_{0}^{t}(\Psi(t-s), \Pi(t-s)) d s\right\|_{-\beta} & \leqslant C \int_{0}^{t} \frac{\|Q(s) \cdot \nabla \rho\|_{-\beta}}{(1+(t-s))^{3 / 2}} d s \\
& \leqslant C^{\prime} \int_{0}^{t} \frac{d s}{(1+(t-s))^{3 / 2}(1+s)^{3 / 2}} \leqslant \frac{C^{\prime \prime}}{(1+t)^{3 / 2}}
\end{aligned}
$$

по известному свойству убывания свертки. Предложение доказано.

Рассмотрим случай волнового уравнения. Обозначим $F(t)=(\Psi(\cdot, t), \Pi(\cdot, t))$. Построим поля - решения первых двух уравнений из (6.12), где $A$ определен формулой (5.4) с $m=0$. Эти два уравнения имеют вид

$$
\dot{F}(t)=\left(\begin{array}{cc}
v \cdot \nabla & 1 \\
\Delta & v \cdot \nabla
\end{array}\right) F+\left(\begin{array}{c}
0 \\
\nabla \rho \cdot Q(t)
\end{array}\right) .
$$

В силу теоремы 12.3 вектор-функция $Q(t)$ непрерывна и

$$
|Q(t)| \leqslant \frac{C\left(\rho, \bar{v}, d_{\delta}\right)}{(1+t)^{1+\delta}}, \quad t \geqslant 0 .
$$

Тогда предложение 6.7 сводится к следующему.

Предложение 13.3. і) Пусть $Q(t) \in C\left([0, \infty) ; \mathbb{R}^{3}\right)$ u $F_{0} \in \dot{\mathscr{F}}$. Тогда уравнение (13.11) имеет единственное решение $F(t) \in C([0, \infty) ; \dot{\mathscr{F}})$ с начальным условием $F(0)=F_{0}$.

ii) Если $X_{0}=\left(F_{0}, Q_{0}, P_{0}\right) \in \mathscr{E}_{\beta}$ и выполнено убывание (13.12), то соответствуюшие поля также убъвают равномерно по $v$ :

$$
\|F(t)\|_{-(2+\delta)} \leqslant \frac{C(\rho, \bar{v})\left\|F_{0}\right\|_{\beta}}{(1+t)^{1+\delta}}, \quad t \geqslant 0,
$$

при $|v| \leqslant \bar{v}$ с произвольным $\bar{v} \in(0,1)$.

Утверждение і) выводится аналогично случаю уравнения Клейна-Гордона. Докажем утверждение іi).

Лемма 13.4. Для $\widetilde{v}<1$ и $F_{0} \in \mathscr{F}_{\alpha}, \alpha>1$, справедливо следующее убывание:

$$
\left\|W(t) F_{0}\right\|_{-\alpha} \leqslant \frac{C(\alpha, \widetilde{v})}{(1+t)^{\alpha-1}}\left\|F_{0}\right\|_{\alpha}, \quad t \geqslant 0,
$$

для динамической группь $W(t)$, отвечающей модифицированному волновому уравнению $с|v|<\widetilde{v}$.

В случае $v=0$ доказательство содержится в [49]. Для ненулевого $v$ с $|v|<\widetilde{v}$ делается несложная модификация. Утверждение іi) предложения 13.3 следует 
из леммы 13.4 и представления Дюгамеля аналогично случаю уравнения Клейна-Гордона.

В случае уравнения Максвелла мы доказываем существование полей $e(x, t)$, $a(x, t)$ - полевых компонент решения $X(t)$ и устанавливаем их убывание, соответствующее (6.19). Обозначим $F(t)=(e(\cdot, t), a(\cdot, t))$. Построим поля-решения первых двух уравнений системы $\dot{X}(t)=A X(t)$, где $A$ задан формулой (10.19). Эти два уравнения имеют вид

$$
\dot{F}(t)=\left(\begin{array}{cc}
v \cdot \nabla & -\Delta \\
-1 & v \cdot \nabla
\end{array}\right) F+\left(\begin{array}{c}
\Pi(t) \\
0
\end{array}\right), \quad \Pi(t):=\Pi\left(r(t) \nabla \rho v-\rho B_{v} \varphi(t)\right) .
$$

В силу теоремы 12.6 компоненты $r(t)$ и $\varphi(t)$ непрерывны и

$$
|r(t)|+|\varphi(t)| \leqslant \frac{C(\rho, \widetilde{v})\left\|X_{0}\right\|_{\beta}}{(1+t)^{1+\delta}}, \quad t \geqslant 0 .
$$

Теперь доказательство предложения 6.7 сводится к следующему утверждению.

ПреДЛОЖЕНИЕ 13.5. і) Пустъ $r(t), \varphi(t) \in C\left([0, \infty) ; \mathbb{R}^{3}\right)$ u $F_{0} \in \mathscr{F}$. Тогда уравнение (13.15) имеет единственное решение $F(t) \in C[0, \infty ; \mathscr{F})$ с начальнъми условиями $F(0)=F_{0}$.

ii) Если $X_{0}=\left(F_{0} ; r_{0}, \varphi_{0}\right) \in \mathscr{E}_{\beta}$ и выполняется убывание (13.16), то соответствующие поля также убъвают равномерно по $v$ :

$$
\|F(t)\|_{-2-\delta} \leqslant \frac{C(\rho, \widetilde{v})\left\|X_{0}\right\|_{\beta}}{(1+t)^{1+\delta}}, \quad t \geqslant 0
$$

для $|v| \leqslant \widetilde{v}$ с произвольным $\widetilde{v} \in(0 ; 1)$.

Доказательство аналогично случаю волнового уравнения.

\section{Приложение А. Вычисление симплектической формы}

Выведем формулы (4.5)-(4.6) для матрицы $\Omega$. Для $j, l=1,2,3$ из формул (4.3) и (4.1) имеем:

$$
\begin{aligned}
\Omega\left(\tau_{j}, \tau_{l}\right) & =\left\langle\partial_{j} \psi_{v}, \partial_{l} \pi_{v}\right\rangle-\left\langle\partial_{j} \pi_{v}, \partial_{l} \psi_{v}\right\rangle \\
\Omega\left(\tau_{j+3}, \tau_{l+3}\right) & =\left\langle\partial_{v_{j}} \psi_{v}, \partial_{v_{l}} \pi_{v}\right\rangle-\left\langle\partial_{v_{j}} \pi_{v}, \partial_{v_{l}} \psi_{v}\right\rangle \\
\Omega\left(\tau_{j}, \tau_{l+3}\right) & =-\left\langle\partial_{j} \psi_{v}, \partial_{v_{l}} \pi_{v}\right\rangle+\left\langle\partial_{j} \pi_{v}, \partial_{v_{l}} \psi_{v}\right\rangle+e_{j} \partial_{v_{l}} p_{v} .
\end{aligned}
$$

Применив преобразование Фурье $\widehat{\psi}(k):=(2 \pi)^{-3 / 2} \int e^{i k x} \psi(x) d x$ к уравнениям $(3.2)$, получим: $\widehat{\psi}_{v}(k)=-\widehat{\rho}(k) / \widehat{D}(0), \widehat{\pi}_{v}(k)=i(k v) \widehat{\psi}_{v}(k)$, где $\widehat{D}(\lambda)$ определено формулой (10.4). Далее,

$$
\begin{aligned}
\partial_{v_{j}} \widehat{\psi}_{v} & =\frac{2(k v) k_{j} \widehat{\psi}_{v}}{\widehat{D}(0)}, \quad \partial_{v_{j}} \widehat{\pi}_{v}=\frac{i k_{j}\left(k^{2}+m^{2}+(k v)^{2}\right) \widehat{\psi}_{v}}{\widehat{D}(0)}, \\
\partial_{v_{j}} p_{v} & =\frac{e_{j}}{\sqrt{1-v^{2}}}+\frac{v_{j}}{\left(1-v^{2}\right)^{3 / 2}} v=\frac{1}{\nu}\left(E+\frac{1}{\nu^{2}} v \otimes v\right) e_{j}
\end{aligned}
$$


для $j=1,2,3$. Напомним, что $B_{v}=\nu(E-v \otimes v)$. Поскольку $(v \otimes v)^{2}=v^{2}(v \otimes v)$, легко проверить, что $B_{v}$ и $\left(E+\left(1 / \nu^{2}\right) v \otimes v\right) / \nu$ являются обратными операторами. Тогда $\partial_{v_{j}} p_{v}=B_{v}^{-1} e_{j}$. Поэтому для $j, l=1,2,3$ с учетом равенства Парсеваля получаем: $\Omega\left(\tau_{j}, \tau_{l}\right)=-2 i \int k_{j} k_{l}(k v)\left|\widehat{\psi}_{v}\right|^{2} d k=0$, поскольку подынтегральная функция нечетна по $k$. Аналогично,

$$
\Omega\left(\tau_{j+3}, \tau_{l+3}\right)=-4 i \int \frac{k_{j} k_{l}(k v)\left(k^{2}+m^{2}+(k v)^{2}\right)\left|\widehat{\psi}_{v}\right|^{2}}{\widehat{D}^{2}(0)} d k=0 .
$$

Наконец,

$$
\Omega\left(\tau_{j}, \tau_{l+3}\right)=\int d k \frac{\left|\widehat{\psi}_{v}\right|^{2} k_{j} k_{l}\left(k^{2}+m^{2}+3(k v)^{2}\right)}{\widehat{D}(0)}+e_{j} \cdot\left(\frac{e_{l}}{\sqrt{1-v^{2}}}+\frac{v_{l} v}{\left(1-v^{2}\right)^{3 / 2}}\right) .
$$

Формулы (4.5), (4.6) получены.

Для случая волнового уравнения те же самые вычисления проводятся с подстановкой $m=0$. При этом интегралы сходятся в силу условия (1.10).

Теперь вычислим матричные элементы $\Omega\left(\tau_{j}, \tau_{l}\right)$ матрицы $\Omega$ в случае уравнения Максвелла и покажем, что матрица невырождена. При $j, l=1,2,3$ из (4.12) и (4.2) следует

$$
\begin{aligned}
\Omega\left(\tau_{j}, \tau_{l}\right) & =\left\langle\partial_{j} E_{v}, \partial_{l} A_{v}\right\rangle-\left\langle\partial_{j} A_{v}, \partial_{l} E_{v}\right\rangle \\
\Omega\left(\tau_{j+3}, \tau_{l+3}\right) & =\left\langle\partial_{v_{j}} E_{v}, \partial_{v_{l}} A_{v}\right\rangle-\left\langle\partial_{v_{j}} A_{v}, \partial_{v_{l}} E_{v}\right\rangle \\
\Omega\left(\tau_{j}, \tau_{l+3}\right) & =-\left\langle\partial_{j} E_{v}, \partial_{v_{l}} A_{v}\right\rangle+\left\langle\partial_{j} A_{v}, \partial_{v_{l}} E_{v}\right\rangle+e_{j} \cdot \partial_{v_{l}} P_{v} .
\end{aligned}
$$

В представлении Фурье солитоны имеют вид

$$
\begin{gathered}
\widehat{E}_{v}(k)=\frac{i(k v) \widehat{\rho}}{D}\left(\frac{(k v)}{k^{2}} k-v\right), \quad \widehat{A}_{v}(k)=\frac{-\widehat{\rho}}{D}\left(\frac{(k v)}{k^{2}} k-v\right), \\
P_{v}=p_{v}+v \int \frac{|\widehat{\rho}|^{2} d k}{D}-\int \frac{|\widehat{\rho}|^{2} d k}{k^{2} D}(k v) k,
\end{gathered}
$$

где $D:=k^{2}-(k v)^{2} ; D$ неотрицательно и четно по $k$. При $j=1,2,3$ имеем:

$$
\begin{aligned}
\partial_{v_{j}} \widehat{E}_{v}= & \frac{i \widehat{\rho}}{D}\left(\frac{2 k_{j}(k v)}{D} k-\frac{k_{j}\left(k^{2}+(k v)^{2}\right)}{D} v-(k v) e_{j}\right) \\
\partial_{v_{l}} \widehat{A}_{v}= & \frac{\widehat{\rho}}{D}\left(\frac{2 k_{l}(k v)}{D} v-\frac{k_{l}\left(k^{2}+(k v)^{2}\right)}{k^{2} D} k+e_{l}\right) \\
\partial_{v_{l}} P_{v}= & \partial_{v_{l}} p_{v}+\left\langle\partial_{v_{l}} A_{v}, \rho\right\rangle=B_{v}^{-1} e_{l}+\int \frac{|\widehat{\rho}|^{2} d k}{D} e_{l}+2 \int \frac{|\widehat{\rho}|^{2}(k v) k_{l} d k}{D^{2}} v \\
& -\int \frac{|\widehat{\rho}|^{2}\left(k^{2}+(k v)^{2}\right) k_{l} d k}{k^{2} D^{2}} k
\end{aligned}
$$

Тогда для $j, l=1,2,3$ по равенству Парсеваля получаем: $\left\langle\partial_{j} E_{v}, \partial_{l} A_{v}\right\rangle=0$, $\left\langle\partial_{j} A_{v}, \partial_{l} E_{v}\right\rangle=0$, и, таким образом, $\Omega\left(\tau_{j}, \tau_{l}\right)=0$. Аналогично, $\left\langle\partial_{v_{j}} E_{v}, \partial_{v_{l}} A_{v}\right\rangle=0$, поскольку подынтегральная функция оказывается нечетной по $k$ и интеграл 
сходится в силу условия (1.10). Так же получаем $\left\langle\partial_{v_{j}} A_{v}, \partial_{v_{l}} E_{v}\right\rangle=0$, и, таким образом, $\Omega\left(\tau_{j+3}, \tau_{l+3}\right)=0$. Теперь вычислим $\Omega\left(\tau_{j}, \tau_{l+3}\right)$. Во-первых,

$$
-\left\langle\partial_{j} E_{v}, \partial_{v_{l}} A_{v}\right\rangle=\int \frac{k_{j}(k v) \mid \widehat{\rho}^{2} d k}{D^{2}}\left(v_{l}+\frac{2 k_{l}(k v) v^{2}}{D}-\frac{k_{l}(k v)\left(k^{2}+(k v)^{2}\right)}{k^{2} D}\right) .
$$

Далее,

$$
\left\langle\partial_{j} A_{v}, \partial_{v_{l}} E_{v}\right\rangle=\int \frac{k_{j}|\widehat{\rho}|^{2} d k}{D^{2}}\left((k v) v_{l}+\frac{k_{l}\left(k^{2}+(k v)^{2}\right) v^{2}}{D}-\frac{2 k_{l}(k v)^{2}}{D}\right) .
$$

Наконец,

$$
\begin{gathered}
e_{j} \cdot \partial_{v_{l}} P_{v}=e_{j} \cdot B_{v}^{-1} e_{l}+\int \frac{|\widehat{\rho}|^{2} d k}{D} \delta_{j l}+2 \int \frac{|\widehat{\rho}|^{2}(k v) k_{l} d k}{D^{2}} v_{j} \\
-\int \frac{|\widehat{\rho}|^{2}\left(k^{2}+(k v)^{2}\right) k_{j} k_{l} d k}{k^{2} D^{2}}
\end{gathered}
$$

Прямым вычислением получаем, что матрица $\Omega^{+}(v)=\left.\left\|\Omega\left(\tau_{j}, \tau_{l+3}\right)\right\|\right|_{j, l=1,2,3}$ положительно определена, а значит, невырождена. В итоге матрица

$$
\left.\left\|\Omega\left(\tau_{j}, \tau_{l}\right)\right\|\right|_{j, l=1, \ldots, 6}=\left(\begin{array}{cc}
0 & \Omega^{+}(v) \\
-\Omega^{+}(v) & 0
\end{array}\right)
$$

также невырождена.

\section{Приложение В. Структура матрицы $M^{-1}(i \omega)$}

Напомним, что при $\omega \in \mathbb{R}$

$$
M(i \omega)=\left(\begin{array}{cc}
i \omega E & -B_{v} \\
-F(i \omega) & i \omega E
\end{array}\right),
$$

где $B_{v}=\operatorname{diag}\left(\nu^{3}, \nu, \nu\right), F(i \omega)=\operatorname{diag}\left(f_{1}(\omega), f(\omega), f(\omega)\right)$. Здесь $f_{1}(\omega)=F_{11}(i \omega+$ $0), f(\omega)=F_{22}(i \omega+0)=F_{33}(i \omega+0) \mathrm{c}$

$$
F_{j j}(\lambda)=\int d k \frac{|\widehat{\rho}|^{2} k_{j}^{2}}{k^{2}+m^{2}+\left(\lambda+i k_{1} v\right)^{2}}-\int d k \frac{|\widehat{\rho}|^{2} k_{j}^{2}}{k^{2}+m^{2}-\left(k_{1} v\right)^{2}} .
$$

По лемме $10.3, F_{j j}(\lambda)$ - аналитические функции на $\mathbb{C}$. Тем самым, $F_{j j}(\lambda)=$ $F_{j j}(0)+F_{j j}^{\prime}(0) \lambda+F_{j j}^{\prime \prime}(0) \lambda^{2} / 2+\cdots$. Ввиду $\left(\right.$ В.1), $F_{j j}(0)=F_{j j}^{\prime}(0)=0$. Тогда $F_{j j}(\lambda)=\lambda^{2} I_{j}(\lambda)$, где $I_{j}(\lambda)$ аналитичны на $\mathbb{C}$. Заметим, что $I_{j}(0)=F_{j j}^{\prime \prime}(0) / 2$. $\mathrm{B}$ итоге

$$
F_{j j}(i \omega)=-\omega^{2} I_{j}(\omega), \quad I_{j}(0)=-\int d k k_{j}^{2}|\widehat{\rho}|^{2} \frac{k^{2}+3\left(v k_{1}\right)^{2}}{\left(k^{2}+m^{2}-\left(v k_{1}\right)^{2}\right)^{3}} .
$$

Обозначим $I(\omega)=I_{2}(\omega)=I_{3}(\omega)$. Прямым вычислением находим обратную матрицу $L(\omega):=M^{-1}(i \omega)$ :

$$
L(\omega)=\left(\begin{array}{cc}
\frac{1}{\omega} L_{11}(\omega) & \frac{1}{\omega^{2}} L_{12}(\omega) \\
L_{21}(\omega) & \frac{1}{\omega} L_{22}(\omega)
\end{array}\right),
$$


где

$$
L_{11}(\omega)=(-i) \operatorname{diag}\left(\frac{1}{1-\nu^{3} I_{1}(\omega)}, \frac{1}{1-\nu I(\omega)}, \frac{1}{1-\nu I(\omega)}\right),
$$

$L_{11}(\omega)=i L_{12}(\omega) B_{v}^{-1}$. Далее,

$$
L_{21}=\operatorname{diag}\left(\frac{I_{1}(\omega)}{1-\nu^{3} I_{1}(\omega)}, \frac{I(\omega)}{1-\nu I(\omega)}, \frac{I(\omega)}{1-\nu I(\omega)}\right),
$$

$L_{22}(\omega)=L_{11}(\omega)$. Заметим, что знаменатели матричных элементов каждой из матриц от $L_{11}$ до $L_{22}$ не равны нулю при $\omega=0$, поскольку $I_{1}(0)<0$ и $I(0)<0$ согласно (В.2). Это завершает доказательство леммы 11.8. Для случая волнового уравнения все вычисления сохраняются с подстановкой $m=0$. В случае уравнения Максвелла имеем

$$
M(i \omega)=\left(\begin{array}{cc}
i \omega E & -B_{v} \\
C(i \omega)+G & i \omega E-F(i \omega)
\end{array}\right)
$$

где $C(i \omega)=\operatorname{diag}\left(c_{1}(i \omega), c(i \omega), c(i \omega)\right), G=\operatorname{diag}\left(g_{1}, g, g\right), F(i \omega)=\operatorname{diag}\left(f_{1}(i \omega)\right.$, $f(i \omega), f(i \omega))$. Здесь $\nu=\sqrt{1-v^{2}}$ и $c_{1}, c, f_{1}, f, g_{1}, g$ заданы формулами (10.26), $(10.34),(10.33)$. Далее, $\operatorname{det} M(i \omega)=d_{1} d^{2}$, где $d_{1}=i \omega\left(i \omega-f_{1}\right)+\nu^{3}\left(c_{1}+g_{1}\right)$, $d=i \omega(i \omega-f)+\nu(c+g)$. Аналогично случаю уравнения Клейна-Гордона получаем, что $c_{1}(i \omega)+g_{1}=-\omega^{2} I_{1}(i \omega), c(i \omega)+g=-\omega^{2} I(i \omega), f_{1}(i \omega)=i \omega J_{1}(i \omega)$, $f(i \omega)=i \omega J(i \omega)$, где $I_{1}, I, J_{1}, J$ - аналитические функции на всей комплексной плоскости. Тогда $d_{1}(i \omega)=-\omega^{2}\left(1-J_{1}(i \omega)+\nu^{3} I_{1}(i \omega)\right), d(i \omega)=-\omega^{2}(1-J(i \omega)+$ $\nu I(i \omega))$. Обратная матрица имеет вид

$$
M^{-1}(i \omega)=\left(\begin{array}{cc}
\frac{1}{\omega} L_{11} & \frac{1}{\omega^{2}} L_{12} \\
L_{21} & \frac{1}{\omega} L_{22}
\end{array}\right)
$$

где (опустим зависимость от $i \omega$ для упрощения обозначений)

$$
\begin{aligned}
& L_{11}=\operatorname{diag}\left(\frac{i\left(J_{1}-1\right)}{1-J_{1}+\nu^{3} I_{1}}, \frac{i(J-1)}{1-J+\nu I}, \frac{i(J-1)}{1-J+\nu I}\right), \\
& L_{12}=\operatorname{diag}\left(\frac{-\nu^{3}}{1-J_{1}+\nu^{3} I_{1}}, \frac{-\nu}{1-J+\nu I}, \frac{-\nu}{1-J+\nu I}\right), \\
& L_{21}=\operatorname{diag}\left(\frac{-I_{1}}{1-J_{1}+\nu^{3} I_{1}}, \frac{-I}{1-J+\nu I}, \frac{-I}{1-J+\nu I}\right), \\
& L_{22}=\operatorname{diag}\left(\frac{-i}{1-J_{1}+\nu^{3} I_{1}}, \frac{-i}{1-J+\nu I}, \frac{-i}{1-J+\nu I}\right) .
\end{aligned}
$$

Заметим, что

$$
L_{11}(\omega)=i L_{12}(\omega) B_{v}^{-1}+i L_{3}
$$


где $L_{3}=\operatorname{diag}\left(\frac{J_{1}}{1-J_{1}+\nu^{3} I_{1}}, \frac{J}{1-J+\nu I_{1}}, \frac{J}{1-J+\nu I_{1}}\right)$. Наконец, убедимся, что знаменатели матричных элементов каждой из матриц от $L_{11}$ до $L_{22}$, а также матрицы $L_{3}$ не обращаются в нуль при $\omega=0$. Для этого достаточно проверить, что $-J_{1}(0)+\nu^{3} I_{1}(0) \geqslant 0$ и $-J(0)+\nu I(0) \geqslant 0$; это делается прямым вычислением.

\section{Список литературы}

[1] H. A. Lorentz, Versuch einer Theorie der electrischen und optischen Erscheinungen in bewegten Körpern, E. J. Brill, Leiden, 1895, 139 pp.

[2] Г. А. Лорентц, Теория электронов и ее применение $к$ явлениям света и теплового излучения, ГИТТЛ, М., 1953; англ. изд.: Н. А. Lorentz, The theory of electrons and its applications to the phenomena of light and radiant heat, reprint of the 2nd ed., Dover Publications, Inc., New York, 1953, 343 pp.

[3] Д. К. Максвелл, “Динамическая теория электромагнитного поля”, Избранные сочинения по теории электромагнитного поля, Классики естествознания, Гостехиздат, М., 1952, 251-341; пер. с англ.: J. C. Maxwell, "A dynamical theory of the electromagnetic field", Philos. Trans. R. Soc. Lond., 155 (1865), 459-512.

[4] E. Wiechert, "Elektrodynamische Elementargesetze", Arch. Néerl. (2), 5 (1900), 549-573.

[5] M. Abraham, Theorie der Elektrizität, v. 2: Elektromagnetische Theorie der Strahlung, B. G. Teubner, Leipzig, 1905, 405 pp.

[6] A. Komech, E. Kopylova, D. Stuart, "On asymptotic stability of solitons in a nonlinear Schrödinger equation", Commun. Pure Appl. Anal., 11:3 (2012), 1063-1079.

[7] A. I. Komech, E. A. Kopylova, H. Spohn, "Scattering of solitons for Dirac equation coupled to a particle", J. Math. Anal. Appl., 383:2 (2011), 265-290.

[8] V. Imaikin, A. Komech, N. Mauser, "Soliton-type asymptotics for the coupled Maxwell-Lorentz equations", Ann. Henri Poincaré, 5:6 (2004), 1117-1135.

[9] H. Spohn, Dynamics of charged particles and their radiation field, Cambridge Univ. Press, Cambridge, 2004, xvi+360 pp.

[10] V.S. Buslaev, G.S. Perel'man, "On the stability of solitary waves for nonlinear Schrödinger equations", Nonlinear evolution equations, Amer. Math. Soc. Transl. Ser. 2, 164, Amer. Math. Soc., Providence, RI, 1995, 75-98.

[11] V.S. Buslaev, C. Sulem, "On asymptotic stability of solitary waves for nonlinear Schrödinger equations", Ann. Inst. H. Poincaré Anal. Non Linéaire, 20:3 (2003), 419-475.

[12] S. Cuccagna, "Stabilization of solutions to nonlinear Schrödinger equations", Comm. Pure Appl. Math., 54:9 (2001), 1110-1145.

[13] S. Cuccagna, "On asymptotic stability of ground states of NLS", Rev. Math. Phys., 15:8 (2003), 877-903.

[14] A. Soffer, M. I. Weinstein, "Resonances, radiation damping and instability in Hamiltonian nonlinear wave equations", Invent. Math., 136:1 (1999), 9-74.

[15] A. Soffer, M.I. Weinstein, "Selection of the ground state for nonlinear Schrödinger equations", Rev. Math. Phys., 16:8 (2004), 977-1071.

[16] A. Komech, H. Spohn, M. Kunze, "Long-time asymptotics for a classical particle interacting with a scalar wave field", Comm. Partial Differential Equations, 22:1-2 (1997), 307-335.

[17] A.I. Komech, H. Spohn, "Soliton-like asymptotics for a classical particle interacting with a scalar wave field", Nonlinear Anal., 33:1 (1998), 13-24. 
[18] A. Komech, M. Kunze, H. Spohn, "Effective dynamics for a mechanical particle coupled to a wave field", Comm. Math. Phys., 203:1 (1999), 1-19.

[19] A.I. Komech, H. Spohn, "Long-time asymptotics for the coupled Maxwell-Lorentz equations", Comm. Partial Differential Equations, 25:3-4 (2000), 559-584.

[20] V. Imaikin, A. Komech, P. Markowich, "Scattering of solitons of the Klein-Gordon equation coupled to a classical particle", J. Math. Phys., 44:3 (2003), 1202-1217.

[21] V. Imaikin, A. Komech, H. Spohn, "Scattering theory for a particle coupled to a scalar field", Discrete Contin. Dyn. Syst., 10:1-2 (2003), 387-396.

[22] V. Imaikin, A. Komech, H. Spohn, "Soliton-type asymptotics and scattering for a charge coupled to the Maxwell field", Russ. J. Math. Phys., 9:4 (2002), 428-436.

[23] P. Germain, "Finite energy scattering for the Lorentz-Maxwell equation", Ann. Henri Poincaré, 9:5 (2008), 927-943.

[24] V. Imaikin, A. Komech, H. Spohn, "Rotating charge coupled to the Maxwell field: scattering theory and adiabatic limit", Monatsh. Math., 142:1-2 (2004), 143-156.

[25] V. Imaikin, A. Komech, B. Vainberg, "On scattering of solitons for the Klein-Gordon equation coupled to a particle", Comm. Math. Phys., 268:2 (2006), 321-367.

[26] V. Imaikin, A. Komech, B. Vainberg, "On scattering of solitons for wave equation coupled to a particle", Probability and mathematical physics, CRM Proc. Lecture Notes, 42, Amer. Math. Soc., Providence, RI, 2007, 249-271.

[27] V. Imaikin, A. Komech, B. Vainberg, "Scattering of solitons for coupled wave-particle equations", J. Math. Anal. Appl., 389:2 (2012), 713-740.

[28] V. Imaikin, A. Komech, H. Spohn, "Scattering asymptotics for a charged particle coupled to the Maxwell field", J. Math. Phys., 52:4 (2011), 042701, 33 pp.

[29] A. Soffer, M.I. Weinstein, "Multichannel nonlinear scattering for nonintegrable systems", Integrable systems and applications (Île d'Oléron, 1988), Lecture Notes in Phys., 342, Springer, Berlin, 1989, 312-327.

[30] A. Soffer, M. I. Weinstein, "Multichannel nonlinear scattering for nonintegrable equations", Comm. Math. Phys., 133:1 (1990), 119-146.

[31] A. Soffer, M. I. Weinstein, "Multichannel nonlinear scattering for nonintegrable equations. II. The case of anisotropic potentials and data", J. Differential Equations, 98:2 (1992), 376-390.

[32] A. Soffer, M.I. Weinstein, "Theory of nonlinear dispersive waves and selection of the ground state", Phys. Rev. Lett., 95:21 (2005), 213905, 4 pp.

[33] V.S. Buslaev, G.S. Perel'man, "On nonlinear scattering of states which are close to a soliton", Méthodes semi-classiques, v. 2 (Nantes, 1991), Astérisque, 210, Société Mathématique de France, Paris, 1992, 49-63.

[34] В. С. Буслаев, Г. С. Перельман, "Рассеяние для нелинейного уравнения Шрёдингера: состояния, близкие к солитону”, Алгебра и анализ, 4:6 (1992), 63-102; англ. пер.: V.S. Buslaev, G. S. Perelman, "Scattering for the nonlinear Schrödinger equation: states that are close to a soliton", St. Petersburg Math. J., 4:6 (1993), 1111-1142.

[35] R. L. Pego, M. I. Weinstein, "On asymptotic stability of solitary waves", Phys. Lett. A, 162:3 (1992), 263-268.

[36] R. L. Pego, M. I. Weinstein, "Asymptotic stability of solitary waves", Comm. Math. Phys., 164:2 (1994), 305-349.

[37] M. I. Weinstein, "Modulational stability of ground states of nonlinear Schrödinger equations", SIAM J. Math. Anal., 16:3 (1985), 472-491.

[38] H. Beresticky, P. L. Lions, "Nonlinear scalar field equations. I. Existence of a ground state", Arch. Ration. Mech. Anal., 82:4 (1983), 313-345; "II. Existence of infinitely many solutions", 347-375. 
[39] M. J. Esteban, V. Georgiev, E. Séré, "Stationary solutions of the Maxwell-Dirac and the Klein-Gordon-Dirac equations", Calc. Var. Partial Differential Equations, 4:3 (1996), 265-281.

[40] M. Grillakis, J. Shatah, W. Strauss, "Stability theory of solitary waves in the presence of symmetry. I", J. Funct. Anal., 74:1 (1987), 160-197; "II", 94:2 (1990), 308-348.

[41] В. И. Арнольд, В.В.Козлов, А. И. Нейштадт, "Математические аспекты классической и небесной механики", Динамические системь - 3, Итоги науки и техники. Соврем. пробл. матем. Фундам. напр., 3, ВИНИТИ, М., 1985, 5-290; англ. пер.: V. I. Arnold, V. V. Kozlov, A. I. Neishtadt, Mathematical aspects of classical and celestial mechanics, Springer-Verlag, Berlin, 1997, xiv+291 pp.

[42] J. R. Miller, M. I. Weinstein, "Asymptotic stability of solitary waves for the regularized long-wave equation", Comm. Pure Appl. Math., 49:4 (1996), 399-441.

[43] А. И. Комеч, “Линейные уравнения в частных производных с постоянными коэффициентами", Дифференциалъные уравнения с частными производными - 2, Итоги науки и техники. Соврем. пробл. матем. Фундам. напр., 31, ВИНИТИ, М., 1988, 127-261; англ. пер.: А. I. Komech, "Linear partial differential equations with constant coefficients", Elements of the modern theory of partial differential equations, Springer, Berlin, 1999, 121-256.

[44] Б. Р. Вайнберг, Асимптотические методы в уравнениях математической физики, Изд-во Моск. ун-та, М., 1982, 295 с.; англ. пер.: В. Vaŭnberg, Asymptotic methods in equations of mathematical physics, Gordon \& Breach Science Publishers, New York, 1989, viii+498 pp.

[45] Б. Р. Вайнберг, "Поведение решения задачи Коши для гиперболического уравнения при $t \rightarrow \infty "$, Матем. сб., 78(120):4 (1969), 542-578; англ. пер.: В. R. Vainberg, "Behavior of the solution of the Cauchy problem for a hyperbolic equation as $t \rightarrow \infty$ ", Math. USSR-Sb., 7:4 (1969), 533-568.

[46] S. Agmon, "Spectral properties of Schrödinger operators and scattering theory", Ann. Scuola Norm. Sup. Pisa Cl. Sci. (4), 2:2 (1975), 151-218.

[47] A. Jensen, T. Kato, "Spectral properties of Schrödinger operators and time-decay of the wave functions", Duke Math. J., 46:3 (1979), 583-611.

[48] Б. Р. Вайнберг, "О коротковолновой асимптотике решений стационарных задач и асимптотике при $t \rightarrow \infty$ решений нестационарных задач", УМH, 30:2(182) (1975), 3-55; англ. пер.: B. R. Vainberg, "On the short wave asymptotic behavior of solutions of stationary problems and the asymptotic behavior as $t \rightarrow \infty$ of solutions of non-stationary problems", Russian Math. Surveys, 30:2 (1975), 1-58.

[49] E. A. Kopylova, "Weighted energy decay for 3D wave equation", Asymptot. Anal., 65:1-2 (2009), 1-16.

В. М. Имайкин (V. M. Imaykin)

Московский институт развития образования

Поступила в редакцию

Департамента образования г. Москвы

E-mail: ivm61@mail.ru

06.02.2013 